Document downloaded from:

http://hdl.handle.net/10251/145198

This paper must be cited as:

Pereira, A.; Santamaría Pérez, D.; Ruiz Fuertes, J.; Manjón, F.; Cuenca Gotor, VP.; Vilaplana Cerda, RI.; Gomis, O.... (26-0). Experimental and Theoretical Study of Bi2O2Se Under Compression. The Journal of Physical Chemistry C. 122(16):8853-8867. https://doi.org/10.1021/acs.jpcc.8b02194

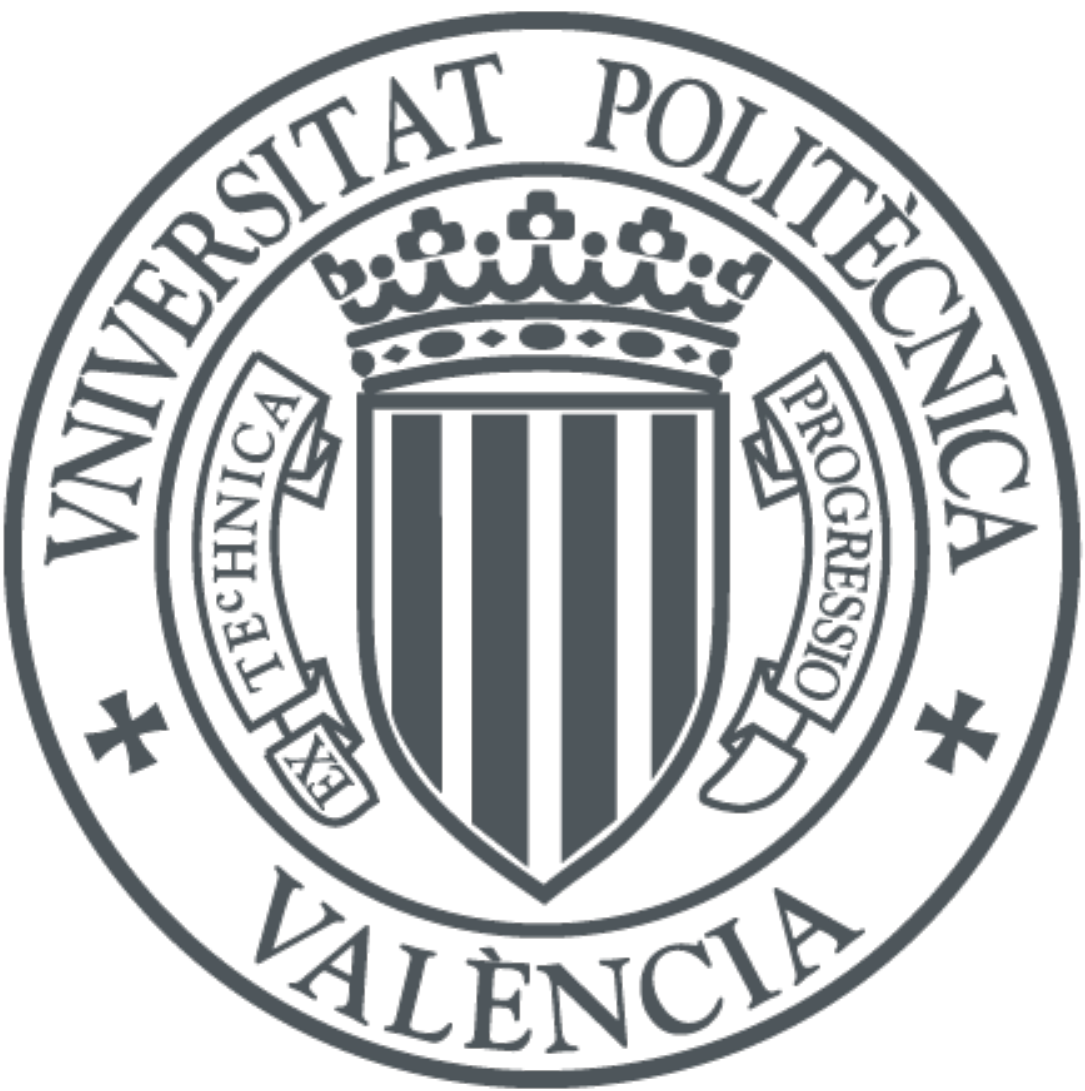

The final publication is available at

https://doi.org/10.1021/acs.jpcc.8b02194

Copyright American Chemical Society

Additional Information 


\title{
Experimental and Theoretical Study of $\mathrm{Bi}_{2} \mathrm{O}_{2} \mathrm{Se}$ Under Compression
}

\author{
A.L.J. Pereira, ${ }^{1,2^{*}}$ D. Santamaría-Pérez, ${ }^{3}$ J. Ruiz-Fuertes, ${ }^{3,4}$ F.J. Manjón, ${ }^{1 *}$ V.P. Cuenca-Gotor, ${ }^{1}$ R. \\ Vilaplana, ${ }^{5}$ O. Gomis, ${ }^{5}$ C. Popescu, ${ }^{6}$ A. Muñoz, ${ }^{7}$ P. Rodríguez-Hernández, ${ }^{7}$ A. Segura, ${ }^{3}$ L. Gracia, ${ }^{8}$ A. \\ Beltrán, ${ }^{9}$ P. Ruleova, ${ }^{10}$ C. Drasar ${ }^{10}$ and J. A. Sans ${ }^{1}$ \\ ${ }^{1}$ Instituto de Diseño para la Fabricación y Producción Automatizada, MALTA Consolider Team, Universitat \\ Politècnica de València, València, Spain \\ ${ }^{2}$ Grupo de Pesquisa de Materiais Fotonicos e Energia Renovável - MaFER, Universidade Federal da \\ Grande Dourados, Dourados, MS, Brazil \\ ${ }^{3}$ Departament de Física Aplicada - ICMUV, MALTA Consolider Team, Universitat de València, Burjassot, \\ Spain \\ ${ }^{4}$ DCITIMAC, MALTA Consolider Team, Universidad de Cantabria, Santander, Spain \\ ${ }^{5}$ Centro de Tecnologías Físicas, MALTA Consolider Team, Universitat Politècnica de València, València, \\ Spain \\ ${ }^{6}$ CELLS-ALBA Synchrotron Light Facility, 08290 Cerdanyola, Barcelona, Spain \\ ${ }^{7}$ Departamento de Física, Instituto de Materiales y Nanotecnología, MALTA Consolider Team, Universidad \\ de La Laguna, Tenerife, Spain \\ ${ }^{8}$ Departament de Química Física, MALTA Consolider Team, Universitat de València, Burjassot, Spain \\ ${ }^{9}$ Departament de Química Física i Analítica, MALTA Consolider Team, Universitat Jaume I, Castellón, \\ Spain \\ ${ }^{10}$ Faculty of Chemical Technology, University of Pardubice, Pardubice, Czech Republic
}

\begin{abstract}
We report a joint experimental and theoretical study of the structural, vibrational, elastic, optical and electronic properties of the layered high-mobility semiconductor $\mathrm{Bi}_{2} \mathrm{O}_{2} \mathrm{Se}$ at high pressure. A good agreement between experiments and ab initio calculations is observed for the equation of state, the pressure coefficients of the Raman-active modes and the bandgap of the material. In particular, a detailed description of the vibrational properties is provided. Unlike other Sillén-type compounds which undergo a tetragonal to collapsed tetragonal pressure-induced phase transition at relatively low pressures, $\mathrm{Bi}_{2} \mathrm{O}_{2} \mathrm{Se}$ shows a
\end{abstract}


remarkable structural stability up to $30 \mathrm{GPa}$; however, our results indicate that this compound exhibits considerable electronic changes around $4 \mathrm{GPa}$, likely related to the progressive shortening and hardening of the long and weak Bi-Se bonds linking the $\mathrm{Bi}_{2} \mathrm{O}_{2}$ and Se atomic layers. Variations of the structural, vibrational, and electronic properties induced by these electronic changes are discussed.

*Corresponding authors, Email: andrepereira@ufgd.edu.br, fjmanjon@fis.upv.es

\section{Introduction}

In recent years, studies related to topological insulators (TIs) have attracted the attention of many research groups. TIs are a class of quantum materials in which timereversal symmetry, relativistic effects and an inverted band structure result in the occurrence, in an insulator bulk crystal, of electronic metallic states on the surface which are protected against non-magnetic impurities or defects. Among such materials, layered compounds $\mathrm{Bi}_{2} \mathrm{Se}_{3}, \mathrm{Bi}_{2} \mathrm{Te}_{3}$ and $\mathrm{Sb}_{2} \mathrm{Te}_{3}$ were predicted and found to behave as $\mathrm{Tis}^{1-4}$. It must be stressed that TIs have promising applications in spintronic devices and dissipationless transistors based on the quantum spin Hall effect and quantum anomalous Hall effect ${ }^{5,6}$, as well as to host Majorana particles ${ }^{7}$. In fact, TIs have already found practical applications in advanced magnetoelectronic and optoelectronic devices ${ }^{8,9}$.

It has been recently suggested that there could be a close relationship between TIs and highly-efficient thermoelectric materials $(\mathrm{TMs})^{10}$; therefore the search of new TIs is also important to find new TMs. In this context, the metal chalcogenide semiconductors belonging to the $A_{2} X_{3}(\mathrm{~A}=\mathrm{Sb}, \mathrm{Bi} ; \mathrm{X}=\mathrm{Se}, \mathrm{Te})$ family with layered tetradymite structure have been of great interest mainly because of their exceptional thermoelectric (TE) properties, with $\mathrm{Bi}_{2} \mathrm{Te}_{3}$ and $\mathrm{Sb}_{2} \mathrm{Te}_{3}$ being the most widely used $\mathrm{TMs}$ at ambient conditions $^{11,12}$.

The strong interest in improving the TE properties of TMs, especially in the most interesting temperature range for TE energy harvest (between 400 and $700 \mathrm{~K}$ ), suggests to engineer the bandgap of the materials since the optimized TMs are predicted to be semiconductors with a bandgap $\mathrm{E}_{\mathrm{g}}=10 \mathrm{k}_{\mathrm{B}} \mathrm{T}_{\mathrm{op}}$, where $\mathrm{k}_{\mathrm{B}}$ is the Boltzmann constant and $\mathrm{T}_{\mathrm{op}}$ is the operating temperature of TE devices ${ }^{13}$. Additionally, there is a strong interest in 
increasing the chemical stability and in decreasing the toxicity of chalcogenide-based TMs. All the above requirements are stimulating the study of layered compounds involving heavy elements such as $\mathrm{Bi}$ and $\mathrm{Sb}$ combined with $\mathrm{O}, \mathrm{S}$ and $\mathrm{Se}$ instead of Te. In this way, a decrease in toxicity and an increase of the chemical stability and of the bandgap will allow to reach the goal of achieving a figure of merit, ZT, near 3 in order to make a widespread use of TMs for TE energy harvest ${ }^{14}$.

Among the studied layered oxychalcogenides, $\mathrm{Bi}_{2} \mathrm{O}_{2} \mathrm{Se}$ has been found as a promising TM since it is an intrinsic n-type semiconductor with rather good TE properties around $800 \mathrm{~K}^{15}$, that can be enhanced under certain stresses, as predicted by ab initio calculations $^{16}$, or by appropriate doping ${ }^{17,18}$. Most importantly, recent studies have shown that $2 \mathrm{D}$ nanometer-size thin films of $\mathrm{Bi}_{2} \mathrm{O}_{2} \mathrm{Se}$ exhibit an extraordinary high-mobility, which combined with its extraordinary chemical stability and large bandgap make this material a promising candidate for the realization of novel quantum phenomena, future logic devices, and flexible electronic applications ${ }^{19,20}$. In fact, the application of $\mathrm{Bi}_{2} \mathrm{O}_{2} \mathrm{Se}$ films in electronic applications has been recently proved ${ }^{21}$. Besides, it has been recently predicted that 2D bismuth oxychalcogenides are exceptional ferroelastic/ferroelectric materials with interest in a number of applications still to be developed ${ }^{22}$.

At room pressure, $\mathrm{Bi}_{2} \mathrm{O}_{2} \mathrm{Se}$ crystallizes in a layered body-centered tetragonal structure (space group No. $139, I 4 / \mathrm{mmm}^{-} \mathrm{D}_{4 \mathrm{~h}}{ }^{17}$ ). In this structure, Se, O and $\mathrm{Bi}$ atoms located at $2 a(0,0,0), 4 d(0,1 / 2,1 / 4)$ and $4 e(0,0, \mathrm{z})$ Wyckoff sites, respectively. Therefore, the $z$ coordinate of the $\mathrm{Bi}$ atom is the only free atomic coordinate in the structure. In this structure (Fig. 1(top)), $\mathrm{Bi}$ and $\mathrm{O}$ atoms form $\mathrm{Bi}_{2} \mathrm{O}_{2}$ layers perpendicular to the [001] direction where $\mathrm{Bi}$ and $\mathrm{O}$ atoms are four-fold coordinated and form a square pyramid with Bi-O bond lengths of $2.3 \AA$. On the other hand, Se atoms form atomic layers located between the $\mathrm{Bi}_{2} \mathrm{O}_{2}$ layers. A weak link between $\mathrm{Bi}_{2} \mathrm{O}_{2}$ and Se atomic layers occurs thanks to weak $\mathrm{Bi}-\mathrm{Se}$ electrostatic interactions (each $\mathrm{Bi}$ atom forms four $\mathrm{Bi}-\mathrm{Se}$ bonds so that each $\mathrm{Se}$ is eightfold coordinated) with a length above 3.0 $\AA$. In summary, the $\mathrm{Bi}_{2} \mathrm{O}_{2} \mathrm{Se}$ structure can be understood as a layered structure where $\mathrm{Bi}_{2} \mathrm{O}_{2}$ layers and $\mathrm{Se}$ atomic layers are $3 \mathrm{D}$ linked thanks to weak Bi-Se electrostatic interactions and where the lone electron pair (LEP) of each $\mathrm{Bi}$ atom is directed perpendicularly to the layers towards the center of the four Se atoms interacting with each Bi atom. In fact, since the closest Bi atoms of different 
layers are aligned along the $c$ axis, their corresponding LEPs are facing to each other across the Se atomic layer.

A way of interpreting the structure of the $\mathrm{Bi}_{2} \mathrm{O}_{2}$ sublattice is by applying the inverse Zintl-Klemm concept ${ }^{23,24}$; i.e., a "Zintl polycation" $\left(\mathrm{Bi}_{2} \mathrm{O}_{2}\right)^{2+}$ is formed when the two electropositive $\mathrm{Bi}$ atoms formally transfer 2 electrons to the $\mathrm{Se}$ atom. In this way, each $\mathrm{Bi}$ atom becomes a pseudo $\Psi-\mathrm{Pb}$ atom and the $\mathrm{Bi}_{2} \mathrm{O}_{2}$ sublattice adopts the same layered structure as litharge $\mathrm{PbO}\left(\mathrm{Pb}_{2} \mathrm{O}_{2} \text { and }\left(\mathrm{Bi}_{2} \mathrm{O}_{2}\right)^{2+} \text { are isoelectronic }\right)^{25}$. This view is consistent with previous reports where $\mathrm{Bi}_{2} \mathrm{O}_{2}$ layers are assumed to be insulating and conductivity of $\mathrm{Bi}_{2} \mathrm{O}_{2} \mathrm{Se}$ is mainly due to the large conductivity along the Se layers ${ }^{19,20}$. It must be stressed that the formation of layered compounds seems to be a common feature in many Sb- and Bi-related sesquioxides and sesquichalcogenides, like the polymorphs $\alpha-\mathrm{Bi}_{2} \mathrm{O}_{3}, \beta-\mathrm{Bi}_{2} \mathrm{O}_{3}$, HPC- $\mathrm{Bi}_{2} \mathrm{O}_{3}, \mathrm{Sb}_{2} \mathrm{~S}_{3}, \mathrm{Bi}_{2} \mathrm{~S}_{3}, \mathrm{Bi}_{2} \mathrm{Se}_{3}, \mathrm{Sb}_{2} \mathrm{Te}_{3}$ and $\mathrm{Bi}_{2} \mathrm{Te}_{3}{ }^{26,27-30}$, and also in other related compounds, like BiSe, BiTe, and $\mathrm{Bi}_{4} \mathrm{Te}_{3}{ }^{31}$.

Pressure is a thermodynamic magnitude and a powerful tool to unravel the intrinsic properties of materials because it is cleaner than chemical doping and simpler to analyze than temperature. Therefore, the understanding of the behavior of $\mathrm{Bi}_{2} \mathrm{O}_{2} \mathrm{Se}$ under compression will help to improve and fully exploit the great potential of the electronic and TE properties of this interesting material. Despite of the increasing interest in bismuth oxychalcogenides, most of the properties of layered $\mathrm{Bi}_{2} \mathrm{O}_{2} \mathrm{Se}$ are barely known specially at high pressures (HPs). In this respect, it must be noted that layered compounds of the $\mathrm{Bi}_{2} \mathrm{Se}_{3}$ family studied at HP have exhibited novel phenomena such as structural phase transitions at low pressures, pressure-induced electronic topological transition (ETT) or Lifshitz transition, and pressure-induced superconductivity ${ }^{26}$. Furthermore, HP exploration of Sillén-type compounds, which are isostructural to $\mathrm{Bi}_{2} \mathrm{O}_{2} \mathrm{Se}$, like iron-based pnictide superconductors, have shown changes in the Fermi surface topology and new superconductor phases usually related to a tetragonal to collapsed tetragonal pressureinduced phase transition at relatively low pressures ${ }^{32-38}$.

In this work, we report a joint experimental and theoretical HP study of the structural, vibrational, elastic, optical, and electronic properties of $\mathrm{Bi}_{2} \mathrm{O}_{2} \mathrm{Se}$ up to $30 \mathrm{GPa}$. No other study of $\mathrm{Bi}_{2} \mathrm{O}_{2} \mathrm{Se}$ under compression has been previously reported to our knowledge. For that purpose, x-ray diffraction (XRD), Raman scattering (RS) and optical 
absorption measurements have been combined with ab initio total-energy structural and lattice-dynamics calculations. It has been found that, unlike other isostructural Sillén-type compounds ${ }^{32-38}$, the tetragonal structure of $\mathrm{Bi}_{2} \mathrm{O}_{2} \mathrm{Se}$ presents a remarkable high stability with no phase transition up to $30 \mathrm{GPa}$. Furthermore, $\mathrm{HP}$ studies of both end members $\mathrm{Bi}_{2} \mathrm{O}_{3}$ and $\mathrm{Bi}_{2} \mathrm{Se}_{3}$ at high pressures show phase transitions below $30 \mathrm{GPa}^{27-29,39}$.

Curiously enough, interesting electronic changes in $\mathrm{Bi}_{2} \mathrm{O}_{2} \mathrm{Se}$ occur near $4 \mathrm{GPa}$ consisting of crossing and anticrossing behaviors of the topmost and second topmost valence bands at different points of the Brillouin zone. These changes are likely related to the progressive shortening and hardening of the long and weak Bi-Se bonds between the layers. Therefore, this study will help to understand the behavior of $\mathrm{Bi}_{2} \mathrm{O}_{2} \mathrm{Se}$ and other Sillén-type compounds under compression and, in general, those of layered materials lacking van der Waals forces between their layers.

\section{Experimental Method}

$\mathrm{Bi}_{2} \mathrm{O}_{2} \mathrm{Se}$ polycrystalline material was recently synthesized from $\mathrm{Bi}_{2} \mathrm{Se}_{3}$ and $\mathrm{Bi}_{2} \mathrm{O}_{3}$ by solid state reaction in an evacuated quartz ampoule ${ }^{40}$. In accordance with the literature, it was observed that the prepared samples crystallize in the space group $139,14 / m m m-\mathrm{D}_{4 \mathrm{~h}}{ }^{17}$, with lattice parameters $a=3.8859 \AA$ and $c=12.2055 \AA$ and yield a unit-cell volume $V_{0}=$ $184.30 \AA^{3}$. The $z$ atomic positions of $\mathrm{Bi}$ and $\mathrm{O}$ are: $z(\mathrm{Bi})=0.355(1)$ and $z(\mathrm{O})=0.250(3)$. All these parameters compare well within $0.2 \%$ with structural of ICSD-2903 entry ${ }^{41}$. This structure is the same as that of $\mathrm{Nd}_{2} \mathrm{O}_{2} \mathrm{Te}^{42}$ and the recently synthesized $\mathrm{Bi}_{2} \mathrm{O}_{2} \mathrm{Te}^{43}$ and it is composed of insulating $\mathrm{Bi}_{2} \mathrm{O}_{2}{ }^{2+}$ layers separated by conducting square $\mathrm{Se}^{2-}$ atomic layers (Fig. 1(top)). Structural analyses have shown no spurious phases or other phases related to $\mathrm{Bi}, \mathrm{O}$ or Se. On the other hand, energy-dispersive X-ray spectroscopy (EDS) analyses showed that the composition of the sample is not purely stoichiometric and has a slight excess of $\mathrm{O}$ and a slight deficiency of $\mathrm{Se}$ with respect to $\mathrm{Bi}$ with an approximate stoichiometry of $\mathrm{Bi}_{2} \mathrm{O}_{2.2} \mathrm{Se}_{0.8}$.

Both powder angle-dispersive XRD measurements at room and HP (up $22 \mathrm{GPa}$ ) were performed at room temperature in an Xcalibur diffractometer with the lines $K_{\alpha 1}$ and $\mathrm{K}_{\alpha 2}$ of a molybdenum source $(\lambda=0.7093$ and $0.7136 \AA$, respectively) with a MerrillBassett-type diamond anvil cell (DAC) with diamond culets of $400 \mu \mathrm{m}$ in diameter ${ }^{44}$. 
Pressure was determined by the luminescence of small ruby chips evenly distributed in the pressure chamber with an error below $0.2 \mathrm{GPa}$ in the whole pressure range studied ${ }^{45}$. A more accurate powder angle-dispersive XRD experiment was also performed up to $21 \mathrm{GPa}$ at room temperature in the BL04-MSPD beamline at ALBA synchrotron facility ${ }^{46}$. This beamline is equipped with Kirkpatrick-Baez mirrors to focus the monochromatic beam and a Rayonix CCD detector with a $165 \mathrm{~mm}$ diameter active area and was operated with a wavelength of $0.4246 \AA$. $\mathrm{Cu}$ powder was used as the internal pressure gauge using the values $V_{0}=47.24 \AA^{3}, B_{0}=133 \mathrm{GPa}$ and $B_{0}{ }^{\prime}=5.01$, corresponding to a $3^{\text {rd }}$-order Vinet equation of state (EOS) which gives a pressure error below $1 \%$ in the $0-20 \mathrm{GPa}$ range ${ }^{47}$. Integration of $2 \mathrm{D}$ diffraction images was performed with Dioptas software ${ }^{48}$ while structural analysis was performed by Le Bail refinements to the XRD patterns recorded at all pressures with Fullprof ${ }^{49}$ and PowderCell ${ }^{50}$ program packages.

Unpolarized HP-RS measurements up to $28.3 \mathrm{GPa}$ were carried out with a Horiba Jobin Yvon LabRAM HR microspectrometer equipped with a thermoelectrically-cooled multichannel charge-coupled device detector which allows a spectral resolution better than $2 \mathrm{~cm}^{-1}$. The signal was collected in backscattering geometry exciting with a HeNe laser $(632.8 \mathrm{~nm}$ line) with a power of less than $10 \mathrm{~mW}$. The measurements were performed at three different zones with different types of filters to assure that the sample was not burned during laser excitation. Nonetheless, it was not observed any significant difference in the spectrum apart from a change in the intensity of the peaks. Pressure was also determined by the ruby luminescence method ${ }^{44}$. Phonons were analyzed by fitting Raman peaks with a Voigt profile fixing the Gaussian linewidth $\left(1.6 \mathrm{~cm}^{-1}\right)$ to the experimental setup resolution.

Finally, HP optical absorption measurements at room temperature up to $19.8 \mathrm{GPa}$ were performed in a plate of $\mathrm{Bi}_{2} \mathrm{O}_{2} \mathrm{Se} 3-\mu \mathrm{m}$-thick and $100 \times 100 \mu \mathrm{m}^{2}$ in size uniformly cleaved along the (001) plane of a single crystal. Therefore, our optical absorption measurements were performed with the electric field geometry $\mathrm{E} \perp c$. The sample was loaded using a mixture of 16:3:1 methanol-ethanol-water together with a ruby chip for pressure calibration in a membrane-type DAC with $500 \mu \mathrm{m}$ diamond culet anvils. The pressure chamber was in a hole of $250 \mu \mathrm{m}$ in diameter made on an Inconel gasket preindented to $45 \mu \mathrm{m}$ in thickness. High-pressure optical absorption experiments at room temperature were performed by the sample-in-sample-out method using a micro-optical 
system ${ }^{51,52}$ composed of a halogen lamp, one $\mathrm{CaF}_{2}$ lens, two Cassegrain objectives, and two spectrometers in the UV-VIS and VIS-NIR range, respectively. It must be stressed that all HP measurements were performed with a 16:3:1 methanol-ethanol-water mixture as a pressure-transmitting medium that provides hydrostatic conditions up to $10 \mathrm{GPa}$ and quasihydrostatic conditions up to the maximum pressure attained in our experiments ${ }^{53}$. Possible effects of non-purely hydrostatic conditions have been described elsewhere ${ }^{54}$.

\section{Computational Details}

$A b$ initio total-energy calculations were performed within the framework of density functional theory (DFT) ${ }^{55}$ to study the structural, vibrational, elastic, optical and electronic properties of $\mathrm{Bi}_{2} \mathrm{O}_{2} \mathrm{Se}$ under pressure. The Vienna Ab-initio Simulation Package (VASP) ${ }^{56}$ was used to carry out simulations with the projector augmented wave $(\mathrm{PAW})^{57}$ pseudopotentials. The PAW scheme replaces core electrons and makes smoothed pseudovalence wave functions taking into account the full nodal character of the allelectron charge density in the core region. The set of plane waves employed was extended up to a kinetic energy cutoff of $520 \mathrm{eV}$ due to the presence of oxygen in the compound under study. The generalized gradient approximation (GGA) was used for the description of the exchange-correlation energy within the PBEsol prescription ${ }^{58}$. The Brillouin zone (BZ) of this body-centered tetragonal structure (with $c>a$ ) was sampled with a dense Monkhorst-Pack grid of special k-points ${ }^{59}$. With the cutoff energy and the k-point sampling employed, a high convergence of 1-2 meV per formula unit in the total energy is achieved. This ensures an accurate calculation of the forces on atoms. At a set of selected volumes, the structure was fully relaxed to the optimized configuration through the calculation of the forces on atoms and the stress tensor until the forces on the atoms were smaller than 0.005 $\mathrm{eV} / \AA$ and the deviations of the stress tensor from a diagonal hydrostatic form were lower than $0.1 \mathrm{GPa}$.

The optimized structure at different pressures was used to study the electronic properties of $\mathrm{Bi}_{2} \mathrm{O}_{2} \mathrm{Se}$ taking into account the spin orbit coupling (SOC). The band structure was analysed along the high-symmetry directions of the BZ. We want to emphasize that the $\mathrm{BZ}$ of $\mathrm{Bi}_{2} \mathrm{O}_{2} \mathrm{Se}$ is that of Sillén-type compounds, like $\mathrm{SrFe}_{2} \mathrm{As}_{2}{ }^{38}$; i.e., that corresponding to a body-centered tetragonal structure (Fig. 1(bottom)) and not the simple tetragonal BZ 
recently used in several calculations of $\mathrm{Bi}_{2} \mathrm{O}_{2} \mathrm{Se}^{19,22,60,61}$. The path suggested for this structure (space group 139, I4/mmm with $\mathrm{c}>\mathrm{a}$ ) by Bradly and Cracknell and used at the Bilbao server web page was used to plot the electronic band structure and phonon dispersion curves (PDCs) ${ }^{62,63}$.

Lattice-dynamics calculations were performed to study the phonons at the $\Gamma$ point of the BZ using the direct force constant approach (or supercell method). The diagonalization of the dynamical matrix provides the frequency and symmetry of the phonon modes. To obtain the PDCs, along high-symmetry directions of the BZ, and the one-phonon density of states (PDOS), similar calculations were performed using appropriate supercells, which allow the PDCs at k-points to be obtained commensurate with the supercell size ${ }^{64}$. Finally, in order to study the HP mechanical stability of $\mathrm{Bi}_{2} \mathrm{O}_{2} \mathrm{Se}$, the elastic stiffness constants were determined employing the stress theorem ${ }^{65}$. The optimized structures were strained, at different pressures, taking into account their symmetry ${ }^{66}$.

\section{Results}

\subsection{Structural properties under pressure}

Angle-dispersive XRD patterns of $\mathrm{Bi}_{2} \mathrm{O}_{2} \mathrm{Se}$ with increasing pressure up to $22 \mathrm{GPa}$ are shown in Figure 2. Le Bail refinements to the XRD patterns recorded at all pressures were performed using the tetragonal $I 4 / \mathrm{mmm}$ structure of $\mathrm{Bi}_{2} \mathrm{O}_{2} \mathrm{Se}$ (see structural data of synchrotron radiation measurements in Table S1 in the Supplementary material). These values at $300 \mathrm{~K}$ are in agreement with our ab initio calculations at $0 \mathrm{~K}$ that underestimate the experimental volume by only $0.5 \%$; i.e., well within the error margin of calculations. Noteworthy, our experimental and theoretical values are also in good agreement with those previously found in the literature ${ }^{15}$.

All diffraction peaks shift to larger angles on increasing pressure up to $22 \mathrm{GPa}$. This result is coherent with the decrease of interplanar distances at HP. No additional diffraction lines or abrupt intensity changes were observed, thus indicating the absence of a structural phase transition. Upon compression, only two interesting phenomena can be noted: i) the overlap of intense peaks related to the (103) and (110) planes above $14.5 \mathrm{GPa}$ and ii) the appearance of a low-intensity peak at $\sim 11^{\circ}$ above $3.0 \mathrm{GPa}$ (Fig. 2). This peak presents a large shift to high angles at HP, remains up to the maximum pressure of our study and is 
not related to the $\mathrm{Bi}_{2} \mathrm{O}_{2} \mathrm{Se}$ structure. In order to find the origin of this peak, we tried to refine the HP-XRD patterns using some possible structures related to elemental $\mathrm{Bi}, \mathrm{O}$ and Se. In this way, this peak was tentatively assigned to the most intense reflections of the $\beta$ $\mathrm{O}_{2}$ and $\varepsilon-\mathrm{O}_{2}$ phases below and above $10 \mathrm{GPa}$, respectively ${ }^{67,68}$. The high compressibility of these solid phases of oxygen would explain the large shift rate to higher angles observed in the measurements and is consistent with the excess of $\mathrm{O}$ found by EDS in original samples. Despite this fact, HP-XRD patterns mainly correspond to the initial tetragonal $\mathrm{Bi}_{2} \mathrm{O}_{2} \mathrm{Se}$ phase up to $22 \mathrm{GPa}$, indicating that $\mathrm{Bi}_{2} \mathrm{O}_{2} \mathrm{Se}$ does not present any clear phase transition. On pressure downstroke, the diffraction pattern obtained after opening the cell is identical to that of the initial sample (see top of Fig. 2). The lack of observation of reflections related to oxygen near room pressure on upstroke and downstroke is consistent with our hypothesis since oxygen is liquid near room conditions and no reflections from a solid phase are expected to be seen.

The pressure dependence of the experimental and theoretical lattice parameters $a$ and $c$ of $\mathrm{Bi}_{2} \mathrm{O}_{2} \mathrm{Se}$ is shown in Fig. 3(left). The axial compressibility, defined as $\kappa_{x}=-\frac{1}{x} \frac{\partial x}{\partial p}$ $(x=a, c)$, obtained from a Birch-Murnaghan (BM) EOS fit of the experimental data ${ }^{69}$ is reported in Table 1 and is in good agreement with our theoretical results. Deviations of experimental lattice parameters and unit cell volume above $10 \mathrm{GPa}$ with respect to theoretical estimates are likely due to non-hydrostatic stresses in the sample due to the loss of hydrostatic conditions of the pressure-transmitting medium ${ }^{53}$. As expected for a $2 \mathrm{D}$ compound, the $c$-axis compressibility is much larger than the $a$-axis compressibility. Moreover, the $c / a$ ratio (inset of Fig. 3(left)) was found to decrease smoothly with pressure in good agreement with our calculations, as it is usual in many layered materials. However, the compressibility of the $c$ axis $\left(6.4 \cdot 10^{-3} \mathrm{GPa}^{-1}\right)$ is much smaller than in other layered materials with van der Waals forces between the layers, like $\mathrm{Bi}_{2} \mathrm{Se}_{3}\left(38.5 \cdot 10^{-3} \mathrm{GPa}^{-1}\right)^{39}$. This result is consistent with the larger strength of weak electrostatic interlayer Bi-Se interactions than of interlayer van der Waals forces. For completeness, the theoretical evolution of the $z$ coordinate of $\mathrm{Bi}$ at HP is given at the inset of Fig. 3(right). It can be observed that there is no clear change of the theoretical $c / a$ ratio or of the $z$ coordinate of $\mathrm{Bi}$ is observed in the range up to $40 \mathrm{GPa}$ that could indicate a second-order pressure-induced 
phase transition from a tetragonal to a collapsed tetragonal structure, unlike in other Silléntype compounds ${ }^{32-38}$.

Figure 3(right) shows the experimental and theoretical pressure dependence of the unit-cell volume of $\mathrm{Bi}_{2} \mathrm{O}_{2} \mathrm{Se}$ up to $22 \mathrm{GPa}$. A $3^{\text {rd }}$-order BM EOS was used to fit our $P-V$ data to obtain the zero pressure volume, $V_{0}$, bulk modulus, $B_{0}$, and its pressure derivative, $B_{0}{ }^{\prime}$, in the quasi-hydrostatic pressure range of the pressure transmitting medium (0-10 GPa). Experimental and theoretical data are summarized in Table 1 showing rather good agreement up to $22 \mathrm{GPa}$, thus the presence of any first-order phase transition can be clearly excluded. It can be stressed that the experimental compressibility of tetragonal $\mathrm{Bi}_{2} \mathrm{O}_{2} \mathrm{Se}$ is found between that of its constituent materials, $\alpha-\mathrm{Bi}_{2} \mathrm{O}_{3}\left(B_{0}=85.4 \mathrm{GPa}^{28}\right)$ and layered $\mathrm{Bi}_{2} \mathrm{Se}_{3}$ $\left(B_{0}=53 \mathrm{GPa}^{39}\right)$. It is noteworthy that the bulk modulus of $\mathrm{Bi}_{2} \mathrm{O}_{2} \mathrm{Se}$ is larger than that of other sesquioxides with channel-type structure, like $\beta-\mathrm{Bi}_{2} \mathrm{O}_{3}\left(B_{0}=34 \mathrm{GPa}^{29}\right)$, and larger than that of layered sesquichalcogenides, like $\mathrm{Bi}_{2} \mathrm{Se}_{3}, \mathrm{Sb}_{2} \mathrm{Te}_{3}\left(B_{0}=40 \mathrm{GPa}^{70}\right)$ and $\mathrm{Bi}_{2} \mathrm{Te}_{3}\left(B_{0}=\right.$ $40.9 \mathrm{GPa}^{71}$ ). These results can be understood by considering that $\mathrm{Bi}_{2} \mathrm{O}_{2} \mathrm{Se}$ is a layered material where $\mathrm{Bi}_{2} \mathrm{O}_{2}$ layers are separated by $\mathrm{Se}$ square arrays showing weak Bi-Se electrostatic interactions at room pressure instead of a standard van der Waals gap between $\mathrm{Bi}_{2} \mathrm{O}_{2}$ layers. As already commented, since the van der Waals gap is much more compressible at low pressures than weak electrostatic interactions, $\mathrm{Bi}_{2} \mathrm{O}_{2} \mathrm{Se}$ shows a smaller compressibility (larger bulk modulus) at room pressure than usual layered compounds with van der Waals gap, like those of the $\mathrm{Bi}_{2} \mathrm{Se}_{3}$ family.

The good agreement between experimental and theoretical data allows us to exploit theoretical data where experimental data are lacking. Since no reliable Rietveld refinement of XRD patterns could be performed (due to texturing effects of the polycrystalline sample) to obtain experimental atomic parameters and bond distances. Figure 4 presents the pressure dependence of the theoretical $\mathrm{Bi}-\mathrm{O}, \mathrm{Bi}-\mathrm{Se}, \mathrm{Se}-\mathrm{O}$ and interlayer $\mathrm{Bi}-\mathrm{Bi}$ distances as well as of the $\mathrm{Bi}_{2} \mathrm{O}_{2}$ layer thickness. As can be observed, the Bi-O bond distance is much smaller than the Bi-Se distance at room pressure, thus indicating the strong ionic-covalent character of the Bi-O bonds and the weak electrostatic character of the Bi-Se bonds. This result justifies the fourfold coordination of $\mathrm{Bi}$ and the $2 \mathrm{D}$ character of $\mathrm{Bi}_{2} \mathrm{O}_{2} \mathrm{Se}$ at room pressure and its easy exfoliation. Regarding the pressure dependence of the bond distances, it can be observed that they decrease with increasing pressure in a monotonous way. 
Notably, while the Bi-O bond distance decreases at a rate of $-4.3 \cdot 10^{-3} \AA / \mathrm{GPa}$ at room pressure, the Bi-Se bond distance decreases more than three times faster $\left(-14.8 \cdot 10^{-3} \AA / \mathrm{GPa}\right)$. This strong decrease of the Bi-Se distance also correlates with the strong decrease of the $\mathrm{Bi}_{2} \mathrm{O}_{2}$ interlayer distance $\left(-29.5 \cdot 10^{-3} \AA / \mathrm{GPa}\right)$ given by the interlayer $\mathrm{Bi}-\mathrm{Bi}$ distance which results in a strong decrease of the $c$ axis $\left(-78.1 \cdot 10^{-3} \AA / \mathrm{GPa}\right)$. The strong decrease of the interlayer distance with pressure contrasts with the negligible decrease of the layer thickness with pressure $\left(-0.2 \cdot 10^{-3} \AA / \mathrm{GPa}\right)$, which in turn is related to the small increase of the $\mathrm{Bi} z$ coordinate. Note that the $\mathrm{Bi}_{2} \mathrm{O}_{2}$ layer thickness decreases at a smaller rate than the $\mathrm{Bi}-\mathrm{O}$ bond distance, thus pointing to an increase of the $\mathrm{Bi}-\mathrm{O}-\mathrm{Bi}$ angle inside the layer with pressure. For comparison with other Sillén-type compounds, the structural values found in $\mathrm{Bi}_{2} \mathrm{O}_{2} \mathrm{Se}$ are between those found for $\mathrm{SrRh}_{2} \mathrm{P}_{2}$ and $\mathrm{BaRh}_{2} \mathrm{P}_{2}$ since the interlayer $\mathrm{Bi}-\mathrm{Bi}$ distance ( $\mathrm{z}$ coordinate of $\mathrm{Bi}$ ) at room pressure is $3.59 \AA(0.354)$ and those for the P-P distance ( $\mathrm{z}$ coordinate of $\mathrm{P}$ ) in $\mathrm{SrRh}_{2} \mathrm{P}_{2}$ and $\mathrm{BaRh}_{2} \mathrm{P}_{2}$ are $3.284 \AA(0.36)$ and $3.737 \AA$ (0.351), respectively ${ }^{32}$. In these two compounds, the decrease of the interlayer P-P distance (equivalent to $\mathrm{Bi}-\mathrm{Bi}$ distance in $\mathrm{Bi}_{2} \mathrm{O}_{2} \mathrm{Se}$ ) is of $-23 \cdot 10^{-3} \AA / \mathrm{GPa}$ which is smaller than in $\mathrm{Bi}_{2} \mathrm{O}_{2} \mathrm{Se}$. A considerable decrease of the interlayer P-P distance occurs in $\mathrm{SrRh}_{2} \mathrm{P}_{2}$ around 6 GPa from a non-bonding to a bonding state, which does not occur in $\mathrm{BaRh}_{2} \mathrm{P}_{2}$ at least up to $11 \mathrm{GPa}$; however, a phase transition at higher pressure (likely around $20 \mathrm{GPa}$ ) is expected when the P-P distance decreases below $3.1 \AA^{32}$. Such bondings are typical of light elements $(\mathrm{O}, \mathrm{S}, \mathrm{N}, \mathrm{P}$ and $\mathrm{As})$, but not of heavy elements, like Bi, so a collapse of the tetragonal structure, like in $\mathrm{SrRh}_{2} \mathrm{P}_{2}, \mathrm{BaRh}_{2} \mathrm{P}_{2}, \mathrm{LaCo}_{2} \mathrm{P}_{2}, \mathrm{EuCo}_{2} \mathrm{P}_{2}, \mathrm{EuFe}_{2} \mathrm{P}_{2}$ and $\mathrm{EuRu}_{2} \mathrm{P}_{2}{ }^{32,33}$, is not expected to occur in $\mathrm{Bi}_{2} \mathrm{O}_{2} \mathrm{Se}$. It remains to be seen what happens in Sb-based Sillén-type compounds 34 .

It must be stressed that despite the strong decrease of the Bi-Se distance, the value of this distance does not decrease below $3.0 \AA$ even up to $30 \mathrm{GPa}$, therefore, despite the BiSe bond length decreases and its strength hardens at HP, Bi maintains its fourfold coordination. Therefore, $\mathrm{Bi}_{2} \mathrm{O}_{2}$ layers are almost unaffected by pressure and the effect of pressure is a rather monotonous and progressive slight contraction of the $\mathrm{Bi}_{2} \mathrm{O}_{2}$ interlayer thickness. In this way, we can affirm that $\mathrm{Bi}_{2} \mathrm{O}_{2}$ Se still behaves as a $2 \mathrm{D}$ material at HP even beyond $30 \mathrm{GPa}$ since there is no net increase of $\mathrm{Bi}$ coordination between room pressure and $30 \mathrm{GPa}$. The progressive and monotonous compression of the interlayer distance contrasts 
with observations in van-der-Waals-based 2D materials, where the strong compression of the interlayer distance at low pressures is followed by a hardening of the interlayer van der Waals interactions and then results in a considerable decrease of the layer thickness. Thus, all our results on the HP behavior of $\mathrm{Bi}_{2} \mathrm{O}_{2} \mathrm{Se}$ are clearly related to the lack of a van der Waals gap between the layers and the presence of a weak electrostatic Bi-Se interaction between the layers in $2 \mathrm{D} \mathrm{Bi}_{2} \mathrm{O}_{2} \mathrm{Se}$.

\subsection{Vibrational properties under pressure}

As regards the lattice dynamics of $\mathrm{Bi}_{2} \mathrm{O}_{2} \mathrm{Se}$, group theoretical considerations of the $\mathrm{Bi}_{2} \mathrm{O}_{2} \mathrm{Se}$ structure in the $I 4 / \mathrm{mmm}$ space group indicates that there are 10 normal modes of vibration at $\Gamma$ whose mechanical decomposition is ${ }^{72}$ :

$$
\Gamma=1 A_{1 g}(R)+2 A_{2 u}(I R)+1 B_{1 g}(R)+2 E_{u}(I R)+2 E_{g}(R)+A_{2 u}+E_{u}
$$

where $A_{1 g}, B_{1 g}$ and $E_{g}$ modes are Raman-active (R) and $A_{2 u}$ and $E_{u}$ are infrared-active (IR). Therefore, there are four Raman-active modes $\left(\Gamma_{\text {Raman }}=\mathrm{A}_{1 \mathrm{~g}}+\mathrm{B}_{1 \mathrm{~g}}+2 \mathrm{E}_{\mathrm{g}}\right)$, four infraredactive (IR) modes $\left(\Gamma_{I R}=2 \mathrm{~A}_{2 \mathrm{u}}+2 \mathrm{E}_{\mathrm{u}}\right)$ and two acoustic modes $\left(\Gamma_{\text {Acoustic }}=\mathrm{A}_{2 \mathrm{u}}+\mathrm{E}_{\mathrm{u}}\right)$. Note that E modes are doubly degenerated. Table 2 summarizes the frequencies of the experimental and theoretical vibrational modes at $\Gamma$ in $\mathrm{Bi}_{2} \mathrm{O}_{2} \mathrm{Se}$ at room pressure together with the assignment of the vibrational modes to atomic movements thanks to the program $\mathrm{J}_{-} \mathrm{ICE}^{73}$. A description of vibrational modes and its comparison with other layered materials is provided in the Supplementary Material.

RS measurements in $\mathrm{Bi}_{2} \mathrm{O}_{2} \mathrm{Se}$ at selected pressures up to $28 \mathrm{GPa}$ (Fig. 5 (left)) show that the most intense Raman peak at room pressure is observed around $159 \mathrm{~cm}^{-1}$ and, according to theoretical calculations, would correspond to the $A_{1 g}$ mode (Fig. 5(right)). The $\mathrm{E}_{\mathrm{g}}$ mode, theoretically predicted at $72.0 \mathrm{~cm}^{-1}$ at room pressure, is not experimentally observed at room pressure because it is in the limit of detection of our system and in a very noisy region of the RS spectrum; however, this peak is clearly seen above $3.8 \mathrm{GPa}$. It must be noted that our RS spectrum at room pressure is similar to that previously reported for bulk samples ${ }^{20}$. As regards the high-frequency $B_{1 \mathrm{~g}}$ and $\mathrm{E}_{\mathrm{g}}$ modes at room pressure, they are theoretically predicted at $369.4 \mathrm{~cm}^{-1}$ and $444.0 \mathrm{~cm}^{-1}$, respectively; however they were not 
observed in our RS spectrum neither it was in previous measurements ${ }^{20}$. Only a broad band near $350 \mathrm{~cm}^{-1}$ is observed which is close to the position expected for the $\mathrm{B}_{1 \mathrm{~g}}$ mode.

At present we have no explanation for the non-observation of the high-frequency Raman-active modes of $\mathrm{Bi}_{2} \mathrm{O}_{2} \mathrm{Se}$ even at room pressure. It could be due to their intrinsically small Raman scattering cross-section (RS measurements with very long exposure time and/or with high laser intensity were also performed) or to phonon damping of longitudinal optic (LO) phonons caused by the large carrier concentration in this n-type semiconductor. In this context, it could be possible that the weak broad bands observed in the highfrequency region could likely be due to plasmon-phonon coupling $\mathrm{L}^{+}$or $\mathrm{L}^{-}$bands of the $\mathrm{B}_{1 \mathrm{~g}}$ and $\mathrm{E}_{\mathrm{g}}$ modes, as it occurs in many highly-doped semiconductors ${ }^{74,75}$. A further study is needed to verify this hypothesis. It is well known that plasmon-phonon coupling severely limits the mobility of carriers ${ }^{76}$, so this coupling could explain the rather low mobility ( $150 \mathrm{~cm}^{2} / \mathrm{Vs}$ ) found in bulk $\mathrm{Bi}_{2} \mathrm{O}_{2} \mathrm{Se}$ at room temperature in comparison to low temperature where carriers are freezed ${ }^{19}$. Additional proofs have to be made in order to resolve this controversy. In case that phonon-plasmon coupling was responsible for the non-observation of high-frequency Raman modes, we propose that observation of high-frequency $B_{1 g}$ and $E_{g}$ Raman-active modes at room pressure could be a good test to monitor the synthesis of $\mathrm{Bi}_{2} \mathrm{O}_{2} \mathrm{Se}$ samples with low carrier concentration in order to improve the mobility of carriers at room temperature.

Since RS spectra of $\mathrm{Bi}_{2} \mathrm{O}_{2} \mathrm{Se}$ up to $28.3 \mathrm{GPa}$ were taken with the same power and exposure time and in the same zone of the sample, we can reasonably compare the absolute intensity of different peaks. In particular, the intensity of the $\mathrm{A}_{1 \mathrm{~g}}$ mode decreases from 0.3 to $4.2 \mathrm{GPa}$ and increases above this pressure with increasing pressure. This change of intensity is likely related to a change of the electronic polarizability due to changes of electronic origin as will be commented in the next section since they cannot be ascribed to structural changes related to the Bi LEP, unlike in other Bi-related compounds as $\beta$ $\mathrm{Bi}_{2} \mathrm{O}_{3}{ }^{29,77}$. Additionally, an increase in the intensity of Raman peaks, followed by the rise of new broad bands (mainly at $154.7 \mathrm{~cm}^{-1}$ and in the region between 250 and $450 \mathrm{~cm}^{-1}$ ), is observed above $10 \mathrm{GPa}$. These new bands were observed in RS measurements performed in different zones of the sample and the bands shift with increasing pressure at a similar rate to that of the first-order Raman modes (see Figure 5(right)), so they cannot be ascribed to 
solid oxygen ${ }^{78}$. In fact, one of them could be related to the $\mathrm{B}_{1 \mathrm{~g}}$ vibrational mode theoretically predicted to be initially at $369.4 \mathrm{~cm}^{-1}$. Since the recovered sample presents the same RS initial spectrum (see top RS spectrum in Fig. 5(left)), it seems that all these bands come from the sample so the hypothesis of sample decomposition is not valid to explain them. Besides, considerable disorder occurs in the sample on increasing pressure above 10 GPa, as will be discussed in the next section. Therefore, we have attributed the broad bands to second-order Raman modes and/or defect-activated Raman scattering corresponding to the PDOS as it occurs in a number of materials ${ }^{75,79,80}$. A comparison of the Raman spectrum at 23.4 GPa and the PDOS at 23.7 GPa (Figure S6 in Supplementary Material) shows that the second hypothesis is reasonable despite there are differences in frequency and intensity between the experimental and theoretical spectra which point to an overestimation of the frequencies of the high-frequency vibrational modes in theoretical calculations. It must be stressed that the RS spectrum of $\mathrm{Bi}_{2} \mathrm{O}_{2} \mathrm{Se}$ after pressure release from $28.3 \mathrm{GPa}$ is similar to the original one. This means that no hint of the high-frequency phonons is observed despite the possible creation of pressure-induced defects, which can behave as electron trapping centers, as it occurs in $\mathrm{ZnO}$ after the highly reconstructive firstorder wurtzite-to-rocksalt transition ${ }^{81}$.

Figure 5(right) shows the experimental and theoretical Raman-active mode frequencies of $\mathrm{Bi}_{2} \mathrm{O}_{2} \mathrm{Se}$ as a function of pressure up to $28.3 \mathrm{GPa}$ and Table 3 summarizes the experimental and theoretical frequencies and pressure coefficients of the vibrational modes of $\mathrm{Bi}_{2} \mathrm{O}_{2} \mathrm{Se}$. As it can be observed, the pressure evolution of the experimental lowfrequency Raman-active $A_{1 g}$ and $E_{g}$ modes agrees well with our calculations despite theoretical frequencies are slightly overestimated. As it can be observed, the relative maxima of the broad band observed above $250 \mathrm{~cm}^{-1}$ also show similar pressure coefficients as the high-frequency modes. It is possible that the clear observation of several vibrational modes especially above $11.3 \mathrm{GPa}$ could be related to the creation of defects in the sample around $10 \mathrm{GPa}$ which also could be due to the loss of hydrostatic conditions of the pressure-transmitting medium and to the reduction of the phonon damping. In this context, more RS studies with samples with different n-type concentration are needed to shed light about the nature of these additional HP vibrational modes. 
In summary, both HP-XRD and HP-RS measurements are in agreement with theoretical calculations and show that $\mathrm{Bi}_{2} \mathrm{O}_{2} \mathrm{Se}$ does not undergo any phase transition up to $30 \mathrm{GPa}$, unlike other isostructural Sillén-type compounds, which undergo a pressureinduced second-order tetragonal-to-collapsed tetragonal phase transition at relatively low pressures $^{32-37}$ and lead to significant changes of vibrational and elastic properties ${ }^{82}$.

\subsection{Dynamical and Mechanical stability of $\mathrm{Bi}_{2} \mathrm{O}_{2} \mathrm{Se}$ at $\mathrm{HP}$}

In order to verify that there is no hidden pressure-induced second-order phase transition, we have obtained the PDCs of $\mathrm{Bi}_{2} \mathrm{O}_{2}$ Se along the main points of the $\mathrm{BZ}$ at 0,12 and 23.7 GPa (Fig. 6). As it can be observed, there are smooth changes of vibrational frequencies with increasing pressure, but there is no softening of vibrational modes (modes whose frequency goes to zero) which could lead to think about the existence of a dynamical instability of the tetragonal phase of $\mathrm{Bi}_{2} \mathrm{O}_{2} \mathrm{Se}$ and therefore the presence of a second-order phase transition in the whole pressure range up to $25 \mathrm{GPa}$.

In a complementary way, we have calculated the pressure dependence of the elastic stiffness constants also known as elastic constants (Figure S7 in Supplementary Material) and studied the generalized stability criteria up to 45 GPa (Fig. 7) for a body-centered tetragonal lattice (see Supplementary Material for more information). Evidence of a mechanical instability of the tetragonal phase of $\mathrm{Bi}_{2} \mathrm{O}_{2} \mathrm{Se}$ is only observed around $41 \mathrm{GPa}$ due to the decrease of the $\mathrm{M}_{2}$ stability criterion. This result clearly shows that the bodycentered tetragonal structure of $\mathrm{Bi}_{2} \mathrm{O}_{2} \mathrm{Se}$ is mechanically stable up to $41 \mathrm{GPa}$.

In a nutshell, we can conclude that our measurements and calculations clearly support the high structural stability of the tetragonal Sillén-type phase of $\mathrm{Bi}_{2} \mathrm{O}_{2} \mathrm{Se}$ under compression and the $2 \mathrm{D}$ character of the compound up to $30 \mathrm{GPa}$. It must be stressed that the high structural stability of body-centered tetragonal $\mathrm{Bi}_{2} \mathrm{O}_{2} \mathrm{Se}$ could be related to the behavior of the Bi LEPs. Bi LEPs, oriented towards the atomic Se layers in $\mathrm{Bi}_{2} \mathrm{O}_{2} \mathrm{Se}$, are likely responsible for the $2 \mathrm{D}$ character of many Bi-containing compounds, as already commented. It has been shown that HP leads to strong compression of Bi LEPs in other Bibased compounds, like $\beta-\mathrm{Bi}_{2} \mathrm{O}_{3}{ }^{29,77}$, and consequently leads to structural changes at relatively low pressures. Therefore, the large structural stability of layered $\mathrm{Bi}_{2} \mathrm{O}_{2} \mathrm{Se}$ can be understood by the relatively low compression of the Bi-Se distance in comparison to the 
decrease of the van der Waals interlayer distance in van-der-Waals-based layered compounds. In this way, there is a small distortion of Bi LEPs at HP that prevents strong structural changes as those observed in other Bi-based compounds.

\subsection{Optical Absorption Measurements}

Despite the interest that TE properties of chalcogenides have attracted, the number of detailed studies regarding their optical properties is scarce. Only very recently, the indirect bandgap of $\mathrm{Bi}_{2} \mathrm{O}_{2} \mathrm{Se}$ has been determined by angle-resolved photoelectron spectroscopy (ARPES) to be around $0.8 \mathrm{eV}^{19}$. Figure 8 shows the optical absorption spectrum of a $3 \mu \mathrm{m}$-thick sample of $\mathrm{Bi}_{2} \mathrm{O}_{2} \mathrm{Se}$ at room conditions. The maximum value that the absorption coefficient reaches $\left(\sim 12 \cdot 10^{3} \mathrm{~cm}^{-1}\right)$ is typical of a direct bandgap. However, the relatively slow rise of the absorption edge that extends for $0.5 \mathrm{eV}$ resembles either the absorption edge of an indirect bandgap, where $\alpha \sim\left(\mathrm{h} v-\mathrm{E}_{\mathrm{g}}\right)^{2}$, or the low-energy exponential absorption tail of a direct transition, as described by the Urbach law ${ }^{83}$. In the case of Urbach's tail, the absorption coefficient can be described by $\alpha=A_{0} \cdot \exp \left[\left(\mathrm{h} v-E_{g}\right) / E_{U}\right]$, where $A_{0}$ is an intrinsic constant of the material, $E_{U}$ is Urbach's energy and relates to the steepness of the absorption edge, and $E_{g}$ is the bandgap. In the inset of Fig. 8, Tauc plots

with the energy dependence of both the $\ln (\alpha)$ and $\alpha^{1 / 2}$ are shown to clarify this issue ${ }^{84,85}$. We find a longer straight linear range with $\ln (\alpha)$ than with $\alpha^{1 / 2}$; thus suggesting that the optical bandgap of $\mathrm{Bi}_{2} \mathrm{O}_{2} \mathrm{Se}$ is most likely direct, with a value $E_{\mathrm{g}}=1.486 \mathrm{eV}$, and its low energy absorption tail follows an Urbach's law. This is also demonstrated by the remarkable agreement between our experimental and simulated spectra (Fig. 8) that provides values of $A_{0}=8300 \mathrm{~cm}^{-1}$ and $E_{U}=0.136 \mathrm{eV}$ once the direct bandgap value is fixed.

The theoretical electronic band structure of bulk $\mathrm{Bi}_{2} \mathrm{O}_{2} \mathrm{Se}$ calculated with DFT GGA-PBEsol including SOC at $0 \mathrm{GPa}$ is shown at the top of Figure 9. Our calculations exhibit the minimum of the conduction band (CB) at the $\Gamma$ point and the maximum of the valence band (VB) at the N point. Other two local maxima of the VB are located along the $\mathrm{Z}-\mathrm{X}-\Gamma$ directions. According to our calculations, the bandgap of $\mathrm{Bi}_{2} \mathrm{O}_{2} \mathrm{Se}$ is indirect $(\mathrm{N}-\Gamma)$ with a value of $0.296 \mathrm{eV}$ and the direct bandgaps of lower energy are located at $\mathrm{Z}(1.13 \mathrm{eV})$ and $\Gamma(1.15 \mathrm{eV})$ points of the BZ. When compared with the indirect bandgap obtained from 
ARPES measurements $(0.8 \mathrm{eV})^{19}$, it can be observed that our calculations underestimate the indirect bandgap by $0.5 \mathrm{eV}$. Taking into account this underestimation of the bandgap, we can estimate from our calculations that the real direct optical transitions are expected around 1.4-1.6 eV; thus in good agreement with our optical absorption measurements at room pressure.

In order to study the HP behavior of the optical bandgap and check the nature of the direct gap, we have performed optical absorption experiments at HP (see Fig. 10). As pressure increases, the absorption edge keeps its shape and shifts to higher energies up to $\sim 5 \mathrm{GPa}$. Above this pressure, the absorption edge becomes insensitive to pressure with the sample keeping its optical quality as evidenced by the persistence of interference fringes at the low-energy range due to the Fabry-Pérot cavity created by the sample. At $\sim 15 \mathrm{GPa}$, in agreement with the appearance of extra vibrational bands observed before by RS spectroscopy, the fringes vanish, the low-energy tail grows, and the edge appears to shift to low energies. This might be indicative of the pressure-induced creation of defects as we will further comment. In summary, the optical bandgap of $\mathrm{Bi}_{2} \mathrm{O}_{2} \mathrm{Se}$ shows a particular behavior that can be divided into three steps. First the bandgap blueshifts up to $5 \mathrm{GPa}$ with a pressure coefficient of $\mathrm{d} E_{g} / \mathrm{d} P=29 \mathrm{meV} / \mathrm{GPa}$. This is followed by a pressure-independent behavior from 5 to $15 \mathrm{GPa}$ and finally a redshift with $\mathrm{d} E_{g} / \mathrm{d} P=-13 \mathrm{meV} / \mathrm{GPa}$, accompanied by an abrupt increase of Urbach's energy.

The HP evolution of the experimental optical bandgap and Urbach's energy $E_{U}$ up to $20 \mathrm{GPa}$ are shown in Fig. 11, where they are compared to the calculated pressure dependence of the indirect $\mathrm{N}-\Gamma$ bandgap and the two lowermost direct $\mathrm{Z}-\mathrm{Z}$ and $\Gamma-\Gamma$ transitions. On one hand, the behavior of the experimental optical bandgap is qualitatively similar to that of the direct bandgap at the $\Gamma$ point. In order to show it more clearly, the theoretical values have been increased by $0.34 \mathrm{eV}$ so that the theoretical direct bandgap at $\Gamma$ matches the experimental value of the optical direct bandgap at $0 \mathrm{GPa}$. On the other hand, the indirect transition shows a maximum at $5 \mathrm{GPa}$ and then slowly redshifts with pressure, while the pressure dependence of the direct $\mathrm{Z}$ transition is also similar to the experimental behavior, but with a much smaller pressure coefficient. Therefore, our results confirm that $\mathrm{Bi}_{2} \mathrm{O}_{2} \mathrm{Se}$ has an indirect bandgap that can be estimated around $\sim 0.8 \mathrm{eV}$ if a shift of $0.5 \mathrm{eV}$ is added to our calculations and a direct optical bandgap around $1.49 \mathrm{eV}$. The reason for not 
observing optically the indirect $\mathrm{N}-\Gamma$ transition and the direct transition at $\mathrm{Z}$ could be related to their forbidden character (the minimum of the $\mathrm{CB}$ is mainly contributed by $\mathrm{p}$ orbitals of Bi) or to their very low absorption coefficient so that the optical direct bandgap at $\Gamma$ is the one observed experimentally.

Above $15 \mathrm{GPa}$, our calculations do not reproduce the redshift experimentally observed (Fig. 11(top)). We believe that the experimentally observed redshiftting tail is due to the progressive creation of defects above $10 \mathrm{GPa}$. The deterioration of the sample above $15 \mathrm{GPa}$ is evident by the disappearance of interference fringes in the optical absorption measurements (Fig. 10), and Urbach's model allows us to indirectly quantify such effect with Urbach's energy $E_{U}$, as shown in Fig. 11(bottom). Up to $15 \mathrm{GPa}, E_{U}$ takes a value of $\sim 160 \mathrm{meV}$, which is already high and similar to that of disordered systems as indium thiospinels ${ }^{86,87}$; however, above $15 \mathrm{GPa}$, the value of $E_{U}$ increases up to $240 \mathrm{meV}$ at 20 $\mathrm{GPa}$, which indicates that the number of defects increases abruptly above $15 \mathrm{GPa}$ and making optical absorption measurements very difficult above $20 \mathrm{GPa}$. Therefore, we ascribe the decrease of the optical bandgap in $\mathrm{Bi}_{2} \mathrm{O}_{2} \mathrm{Se}$ above $15 \mathrm{GPa}$ to the creation of defects that produce levels between the VB and $\mathrm{CB}$, thus decreasing the optical bandgap. In this respect, the decrease of pressure allows to show the reversibility of the change of the optical bandgap, but the sample is so damaged that interference fringes are no longer observed.

It is interesting to notice the change of the pressure dependence exhibited by both the direct and indirect bandgaps between 4 and $5 \mathrm{GPa}$ with an abrupt change of their pressure coefficients. In order to understand these phenomena, we have compared in Fig. 9 the theoretical electronic band structure of bulk $\mathrm{Bi}_{2} \mathrm{O}_{2} \mathrm{Se}$ at 0 and $4.8 \mathrm{GPa}$. The electronic density of states (DOS) at both pressures is also provided as Fig. S9 in the Supplementary material. As observed, the two topmost VBs at $\Gamma$ are closer at $4.8 \mathrm{GPa}$ than at $0 \mathrm{GPa}$. In fact, they cross each other around $4 \mathrm{GPa}$. This crossing explains the abrupt change in pressure coefficient of the direct gap between 4 and $5 \mathrm{GPa}$. This effect can be better seen in Fig. 12(left) where the pressure dependence of the orbital decomposition of the topmost VB (TVBs) and the second topmost VB (STVBs) are shown. At room pressure, the TVB in $\mathrm{Bi}_{2} \mathrm{O}_{2} \mathrm{Se}$ at $\Gamma$ is mainly contributed by the $\mathrm{p}_{\mathrm{x}}$ and $\mathrm{p}_{\mathrm{y}}$ orbitals of Se while the STVB is mainly contributed by the $\mathrm{p}_{z}$ orbital of Se. Since STVB has a larger pressure coefficient than TVB, 
they come close to each other and a crossing of these bands occurs at $4 \mathrm{GPa}$. Above that pressure, the presence of the $p_{x}$ and $p_{y}$ orbitals at the TVB almost decreases to zero and the $p_{z}$ orbital is the main responsible for these bands at higher pressures. The contrary occurs to STVB so the energy distance between TVB and STBV increases above $4 \mathrm{GPa}$. This abrupt change is a clear indication of a band crossing. The larger pressure coefficient of the bands with $\mathrm{p}_{\mathrm{x}}$ and $\mathrm{p}_{\mathrm{y}}$ character is similar to that of the conduction band at $\Gamma$ (also with $\mathrm{p}_{\mathrm{x}}$ and $\mathrm{p}_{\mathrm{y}}$ character) so the pressure coefficient of the direct bangap above $5 \mathrm{GPa}$ becomes negligible.

Similarly, the indirect bandgap $\mathrm{N}-\Gamma$ (Fig. 11) presents a change in the pressure coefficient (a maximum value in energy is obtained around $4.8 \mathrm{GPa}$ ). As in the case of the direct bandgap, the change in pressure coefficient of the $\mathrm{N}-\Gamma$ indirect bandgap can be understood by the change of the character of the two topmost VBs at the $\mathrm{N}$ point with increasing pressure. At ambient pressure, the TVB at the $\mathrm{N}$ point is mainly contributed by $p_{\mathrm{x}}$ and $\mathrm{p}_{\mathrm{y}}$ orbitals of Se (Fig. 12(right)), being the $\mathrm{p}_{\mathrm{x}}$ orbital contribution more than 10 times higher than the $p_{y}$ one. On the other hand, the STVB at the N point is of the same symmetry and has similar orbital contribution of $p_{x}$ and $p_{y}$ orbitals of Se; however, in this case the $\mathrm{p}_{\mathrm{y}}$ orbital contribution around 2.5 times higher than the $\mathrm{p}_{\mathrm{x}}$ one. Since STVB have a larger pressure coefficient than TVB, they become closer in energy with increasing pressure, reaching a minimum energy distance around 5.0 GPa, and tend to separate at higher pressures. This is typical of a band anticrossing since both bands have the same character $^{88}$. At the same time, a progressive variation of the contributions of $p_{x}$ and $p_{y}$ orbitals of Se in both VBs occur as they come closer. In fact, the proportion of $p_{y}$ character in the TVB becomes larger than that of $\mathrm{p}_{\mathrm{x}}$ character above $5.0 \mathrm{GPa}$ and the contrary occurs in the STVB, where the proportion of $p_{\mathrm{x}}$ character becomes larger than that of $p_{\mathrm{y}}$ character above $2.5 \mathrm{GPa}$. These continuous changes of character are indicative of an anticrossing of the two topmost VBs at the N point around 4 GPa. In summary, we can conclude that these crossings and anticrossings observed in the top of the $\mathrm{VB}$ of $\mathrm{Bi}_{2} \mathrm{O}_{2} \mathrm{Se}$ are related to the high compressibility of the $\mathrm{Bi}-\mathrm{Se}$ bonds as compared with the $\mathrm{Bi}-\mathrm{O}$ bonds. Note that the evolution of the Bi-Se distance with pressure (Fig. 4) shows a pressure coefficient of $14.8 \cdot 10^{-3} \AA / \mathrm{GPa}$ in the range from 0 to $4 \mathrm{GPa}$ and a pressure coefficient of $-8.2 \cdot 10^{-3} \AA / \mathrm{GPa}$ in the range of 10 to $16 \mathrm{GPa}$; thus indicating that the bond Bi-Se becomes almost half as compressible at $10 \mathrm{GPa}$ than at room pressure. This is a consequence of the $2 \mathrm{D}$ layered 
character of $\mathrm{Bi}_{2} \mathrm{O}_{2} \mathrm{Se}$ (Fig. 1(top)); therefore, we can ascribe the changes observed near 4 $\mathrm{GPa}$ in RS measurements to changes in the electronic band structure related to the strong compression of the interlayer distance and therefore affecting considerably to Se atoms. Finally, it must be stressed that the change of the character of the TVB at the N point around $4 \mathrm{GPa}$ could lead to a pressure-induced electronic topological transition (ETT) if ptype doping of $\mathrm{Bi}_{2} \mathrm{O}_{2} \mathrm{Se}$ is achieved.

\section{Conclusions}

Our joint experimental and theoretical study of the structural, vibrational, optical, elastic and electronic properties of layered $\mathrm{Bi}_{2} \mathrm{O}_{2} \mathrm{Se}$ under compression has shown a good agreement between experiments and ab initio calculations for the EOS, the pressure coefficients of the Raman-active modes and the bandgap of this material. The compressibility and vibrational properties of this layered compound based on weak interlayer electrostatic interactions has been analyzed and discussed in comparison with typical layered compounds based on van der Waals interlayer forces. In particular, the vibrational properties of $\mathrm{Bi}_{2} \mathrm{O}_{2} \mathrm{Se}$ have been discussed in detail on the light of the correct Brillouin zone for Sillén-type compounds.

No first- or second-order phase transition has been observed in $\mathrm{Bi}_{2} \mathrm{O}_{2} \mathrm{Se}$ up to 30 GPa. Therefore, this material is one of the more stable Sillén-type compounds under compression. Curiously, the $\mathrm{Bi}$-based compound $\mathrm{BiSbO}_{4}$ has been recently found to be stable up to $70 \mathrm{GPa}^{89}$. Despite the lack of important structural changes at high pressure, $\mathrm{Bi}_{2} \mathrm{O}_{2} \mathrm{Se}$ exhibits interesting electronic changes around $4 \mathrm{GPa}$, which are mainly related to the shortening and hardening of the long Bi-Se bonds, and leads to a strong change in the vibrational, optical and electronic properties of the material due to a change of the character of the topmost valence bands. We hope this work will stimulate further studies of $\mathrm{Bi}_{2} \mathrm{O}_{2} \mathrm{Se}$ and will help to shed light over this high-mobility semiconductor in order to enhance the interesting electronic and TE properties of bismuth oxychalcogenides. Furthermore, the results here reported for $\mathrm{Bi}_{2} \mathrm{O}_{2} \mathrm{Se}$ will be helpful to understand the properties of other layered Sillén-type compounds and in general in other layered materials lacking van der Waals forces between their layers. In particular, this study will be interesting to understand 
the behavior under pressure of Sb-based Sillén-type compounds ${ }^{32}$ whose properties under pressure have not been explored to our knowledge.

\section{Acknowledgements}

This work was supported by Brazilian Conselho Nacional de Desenvolvimento Científico e Tecnológico (CNPq) under project 201050/2012-9, by Spanish MINECO projects MAT2015-71070-REDC, MAT2016-75586-C4-1/2/3-P and CTQ2015-65207-P and by the Grant Agency of the Czech Republic (GA CR) under project 16-07711S. Supercomputer time has been provided by the Red Española de Supercomputación (RES) and the MALTA cluster. D.S.-P. and J.A.S. acknowledge the "Ramón y Cajal" fellowship program and J.R.-F. the "Juan de la Cierva" program (IJCI-2014-20513) for financial support.

\section{Supporting Information}

Experimental lattice parameters and unit cell volumes of $\mathrm{Bi}_{2} \mathrm{O}_{2} \mathrm{Se}$ at different pressures. Visualization of the vibrational modes at the $\Gamma$ point. Evolution with pressure of the theoretical infrared active (IR-active) modes, elastic constants, $\mathrm{C}_{\mathrm{ij}}$, and elastic stiffness, $\mathrm{B}_{\mathrm{ij}}$, of $\mathrm{Bi}_{2} \mathrm{O}_{2} \mathrm{Se}$. Electronic Density of States (DOS) of $\mathrm{Bi}_{2} \mathrm{O}_{2} \mathrm{Se}$ at $0 \mathrm{GPa}$ and $4.8 \mathrm{GPa}$.

\section{References}

1. Xia, Y.; Qian, D.; Hsieh, D.; Wray, L.; Pal, A.; Lin, H.; Bansil, A.; Grauer, D.; Hor, Y. S.; Cava, R. J.; et al. Discovery (Theoretical Prediction and Experimental Observation) of a Large-Gap Topological-Insulator Class with Spin-Polarized SingleDirac-Cone on the Surface. Nat. Phys. 2009, 5, 398-402.

2. Zhang, H.; Liu, C. X.; Qi, X. L.; Dai, X.; Fang, Z.; Zhang, S. C. Topological Insulators in $\mathrm{Bi}_{2} \mathrm{Se}_{3}, \mathrm{Bi}_{2} \mathrm{Te}_{3}$ and $\mathrm{Sb}_{2} \mathrm{Te}_{3}$ With a Single Dirac Cone on the Surface. Nat. Phys. 2009, $5,438-442$. 
3. Chen, Y. L.; Analytis, J. G.; Chu, J.-H.; Liu, Z. K.; Mo, S.-K.; Qi, X. L.; Zhang, H. J.; Lu, D. H.; Dai, X.; Fang, Z.; et al. Experimental Realization of a Three-Dimensional Topological Insulator, $\mathrm{Bi}_{2} \mathrm{Te}_{3}$. Science 2009, 325, 178-181.

4. Hsieh, D.; Xia, Y.; Qian, D.; Wray, L.; Meier, F.; Dil, J. H.; Osterwalder, J.; Patthey, A; Fedorov, A. V.; Lin, H.; et al. Observation of Time-Reversal-Protected SingleDirac-Cone Topological-Insulator States in $\mathrm{Bi}_{2} \mathrm{Te}_{3}$ and $\mathrm{Sb}_{2} \mathrm{Te}_{3}$. Phys. Rev. Lett. 2009, $103,146401$.

5. König, M.; Wiedmann, S.; Brüne, C.; Roth, A.; Buhmann, H.; Molenkamp, L. W.; Qi, X.-L.; Zhang, S.-C. Quantum Spin Hall Insulator State in HgTe Quantum Wells. Science 2007, 318, 766-770.

6. Chang, C. -Z.; Zhang, J.; Feng, X.; Shen, J.; Zhang, Z.; Guo, M.; Li, K.; Ou, Y.; Wei, P. Experimental Observation of the Quantum Anomalous Hall Effect in a Magnetic Topological Insulator. Science 2013, 340, 167-170.

7. Fu, L.; Kane, C. L. Superconducting Proximity Effect and Majorana Fermions at the Surface of a Topological Insulator. Phys. Rev. Lett. 2008, 100, 096407.

8. Yue, Z.; Cai, B.; Wang, L.; Wang, X.; Gu, M. Intrinsically Core-Shell Plasmonic Dielectric Nanostructures with Ultrahigh Refractive Index. Sci. Adv. 2016, 2, e1501536.

9. Yue, Z.; Xue, G.; Liu, J.; Wang, Y.; Gu, M. Nanometric Holograms Based on a Topological Insulator Material. Nat. Comm. 2017, 8, ncomms15354.

10. Yang, J. Prospective Thermoelectrics among Topological Insulators, Materials Aspect of Thermoelectricity, ed. by. C. Uher, CRC, Boca Raton (USA), 2017.

11. Rowe, D. M. CRC Handbook of Thermoelectrics, CRC Press Inc., New York, 1995. 
12. Venkatasubramanian, R.; Siivola, E.; Colpitts, T.; O’Quinn, B. Thin-Film Thermoelectric Devices with High Room-Temperature Figures of Merit. Nature 2001, $413,597-602$.

13. Mahan, G. D. Figure of Merit for Thermoelectrics. J. Appl. Phys. 1989, 65, 15781583.

14. Harman, T. C.; Taylor, P. J.; Walsh, M. P.; LaForge, B. E. Quantum Dot Superlattice Thermoelectric Materials and Devices. Science 2002, 297, 2229-2232.

15. Ruleova, P.; Drasar, C.; Lostak, P.; Li, C. P.; Ballikaya, S.; Uher, C. Thermoelectric Properties of $\mathrm{Bi}_{2} \mathrm{O}_{2}$ Se. Mater. Chem. Phys. 2010, 119, 299-302.

16. Guo, D.; Hu, C.; Xi, Y.; Zhang, K. Strain Effects to Optimize Thermoelectric Properties of Doped $\mathrm{Bi}_{2} \mathrm{O}_{2} \mathrm{Se}$ via Tran - Blaha Modified Becke - Johnson Density Functional Theory. J. Phys. Chem. C 2013, 117, 21597-21602.

17. Tan, X.; Lan, J. -L.; Ren, G.; Liu, Y.; Lin, Y. -H.; Nan, C. -W. Enhanced Thermoelectric Performance of N-Type $\mathrm{Bi}_{2} \mathrm{O}_{2} \mathrm{Se}$ by Cl-Doping at Se Site. J. Amer. Chem. Soc. 2017, 100, $1494-1501$.

18. Tan, X.; Liu, Y.; Hu, K.; Ren, G.; Li, Y.; Liu, R.; Lin, Y. -H.; Lan, J. -L.; Nan, C. -W. Synergistically Optimizing Electrical and Thermal Transport Properties of $\mathrm{Bi}_{2} \mathrm{O}_{2} \mathrm{Se}$ Ceramics by Te-Substitution. J. Amer. Ceram. Soc. 2018, 101, 326-333.

19. Wu, J.; Yuan, H.; Meng, M.; Chen, C.; Sun, Y.; Chen, Z.; Dang, W.; Tan, C.; Liu, Y.; Yin, J.; et al. High Electron Mobility and Quantum Oscillations in Non-Encapsulated Ultrathin Semiconducting $\mathrm{Bi}_{2} \mathrm{O}_{2}$ Se. Nat. Nanotech. 2017, 12, 530-534.

20. Wu, J. X.; Tan, C. W.; Tan, Z. J.; Liu, Y. J.; Yin, J. B.; Dang, W. H.; Wang, M. Z.; Peng, H. L. Controlled Synthesis of High-Mobility Atomically Thin Bismuth Oxyselenide Crystals. Nano Letters 2017, 17, 3021-3026. 
21. Wu, J.; Liu, Y.; Tan, Z.; Tan, C.; Yin, J.; Li, T.; Tu, T.; Peng, H. Chemical Patterning of High-Mobility Semiconducting 2D $\mathrm{Bi}_{2} \mathrm{O}_{2} \mathrm{Se}$ Crystals for Integrated Optoelectronic Devices. Advanced Materials 2017, 29, 44.

22. Wu, M.; Zeng, X. C. Bismuth Oxychalcogenides: A New Class of Ferroelectric/Ferroelastic Materials with Ultra High Mobility. Nano Letters 2017, 17, 6309-6314.

23. Shevelkov, A.V.; Shatruk, M. M. Mercury and Cadmium pnictide-halides: The Inverted Zintl Phases. Russ. Chem. Bull. Int. Ed. 2001, 50, 337-352.

24. Vegas, A.; Santamaria-Perez, D. The Structures of $\mathrm{ZrNCl}, \mathrm{TiOCl}$ and $\mathrm{AlOCl}$ in the Light of the Zintl-Klemm Concept. Z. Kristallogr. 2003, 218, 466-469.

25. Leciejewicz, J. On the Crystal Structure of Tetragonal (red) PbO. Acta Cryst. 1961, 14, 1304.

26. Manjón, F. J.; Vilaplana, R.; Gomis, O.; Pérez-González, E.; Santamaría-Rérez, D.; Marín-Borrás, V.; Segura, A.; González, J.; Rodríguez-Hernández, P.; Muñoz, A.; et al. High-pressure Studies of Topological Insulators $\mathrm{Bi}_{2} \mathrm{Se}_{3}, \mathrm{Bi}_{2} \mathrm{Te}_{3}$, and $\mathrm{Sb}_{2} \mathrm{Te}_{3}$. Phys. Stat. Sol. (b) 2013, 250, 669-676.

27. Pereira, A. L. J.; Errandonea, D.; Beltrán, A.; Gracia, L.; Gomis, O.; Sans, J. A.; García-Domene, B.; Miquel-Veyrat, A.; Manjón, F. J.; Muñoz, A.; et al. Structural Study of $\alpha-\mathrm{Bi}_{2} \mathrm{O}_{3}$ under Pressure. J. Phys.: Cond. Mat. 2013, 25, 475402.

28. Pereira, A. L. J.; Gomis, O.; Sans, J. A.; Pellicer-Porres, J.; Manjón, F. J.; Beltran, A.; Rodríguez-Hernandez, P.; Muñoz, A. Pressure Effects on the Vibrational Properties of $\alpha-\mathrm{Bi}_{2} \mathrm{O}_{3}$ : An Experimental and Theoretical Study. J. Phys.: Cond. Mat. 2014, 26, 225401. 
29. Pereira, A. L. J.; Sans, J. A.; Vilaplana, R.; Gomis, O.; Manjón, F. J.; RodríguezHernández, P.; Muñoz, A.; Popescu, C.; Beltrán, A. Isostructural Second-Order Phase Transition of $\beta-\mathrm{Bi}_{2} \mathrm{O}_{3}$ at High Pressures: An Experimental and Theoretical Study. $J$. Phys. Chem. C 2014, 118, 23189.

30. Ibañez, J.; Sans, J. A.; Popescu, C.; López-Vidrier, J.; Elvira-Betanzos, J. J.; CuencaGotor, V. P.; Gomis, O.; Manjón, F. J.; Rodríguez-Hernández, P.; Muñoz, A. Structural, Vibrational, and Electronic Study of $\mathrm{Sb}_{2} \mathrm{~S}_{3}$ at High Pressure. J. Phys. Chem. C 2016, 120, 10547.

31. Loa, I.; Bos, J.-W. G.; Downie, R. A.; Syassen, K. Atomic Ordering in Cubic Bismuth Telluride Alloy Phases at High Pressure. Phys. Rev. B 2016, 93, 224109.

32. Huhnt, C.; Michels, G.; Roepke, M.; Schlabitz, W.; Wurth, A.; Johrendt, D.; Mewis, A. First-Order Phase Transitions in the $\mathrm{ThCr}_{2} \mathrm{Si}_{2}$-type Phosphides $\mathrm{ARh}_{2} \mathrm{P}_{2}(\mathrm{~A}=\mathrm{Sr}, \mathrm{Eu})$. Phys. B: Cond. Matt. 1997, 240, 26-37.

33. Huhnt, C.; Schlabitz, W.; Wurth, A.; Mewis, A.; Reehuis, M. First- and Second-Order Phase Transitions in Ternary Europium Phosphides with $\mathrm{ThCr}_{2} \mathrm{Si}_{2}$-type Structure. Phys. B: Cond. Mat. 1998, 252, 44-54.

34. Li, Y.Y.; Ni, J. The stable $\mathrm{CaBe}_{2} \mathrm{Ge}_{2}$ Structures in Antimonide Compounds $\mathrm{ATM}_{2} \mathrm{Sb}_{2}$ $(\mathrm{A}=\mathrm{Ca}, \mathrm{Sr}, \mathrm{Ba} ; \mathrm{TM}=\mathrm{Fe}, \mathrm{Co}, \mathrm{Ni}, \mathrm{Cu})$ : A First-Principles Study. Phys. Lett. A 2011, $375,4218-4224$.

35. Sefat, A. S. Pressure Effects on Two Superconducting Iron-Based Families. Rep. Prog. Phys. 2011, 74, 124502-124516. 
36. Sun, L.; Chen, X.-J.; Guo, J.; Gao, P.; Huang, Q.-Z.; Wang, H.; Fang, M.; Chen, X.; Chen, G.; Wu, Q.; Zhang, C.; et al. Re-Emerging Superconductivity at 48 Kelvin in Iron Chalcogenides. Nature 2012, 483, 67-69.

37. Das, T.; Balatsky, A. V. Origin of Pressure Induced Second Superconducting Dome in $\mathrm{A}_{\mathrm{y}} \mathrm{Fe}_{2}-\mathrm{xSe}_{2}[\mathrm{~A}=\mathrm{K},(\mathrm{Tl}, \mathrm{Rb})]$. New J. Phys. 2013, 15, 093045-093057.

38. Wang, Y. Q.; Lu, P. C.; Wu, J. J.; Liu, J.; Wang, X. C.; Zhao, J. Y.; Bi, W.; Alp, E. E.; Park, C. Y.; Popov, D.; et al. Phonon Density of States of SingleCrystal $\mathrm{SrFe}_{2} \mathrm{As}_{2}$ across the Collapsed Phase Transition at High Pressure. Phys. Rev. $B$ 2016, 94, 014516.

39. Vilaplana, R.; Santamaría-Pérez, D.; Gomis, O.; Manjón, F. J.; González, J.; Segura, A.; Muñoz, A.; Rodríguez-Hernández, E.; Pérez-González, E.; Marín-Borrás, V.; et al. Structural and Vibrational Study of $\mathrm{Bi}_{2} \mathrm{Se}_{3}$ under High Pressure. Phys. Rev. B 2011, 84, 184110.

40. Ruleova, P.; Drasar, C.; Benes, L.; Lostak, P.; Li, C.-P.; Kong, H.; Uher, C. $\mathrm{Bi}_{2} \mathrm{O}_{2} \mathrm{Se}-$ A Prospective Thermoelectric Material? 6th Eur. Conf. Thermoelectr. 2009, P2-35.

41. Boller, H. Die Kristallstruktur von $\mathrm{Bi}_{2} \mathrm{O}_{2}$ Se. Monatshefte fuer Chemie 1973, 104, 916919.

42. Raccah, P. M.; Longo, J. M.; Eick, H. A. The Crystal Structure of Neodymium Monotellurooxide- $\mathrm{Nd}_{2} \mathrm{O}_{2}$ Te. Inorg. Chem. 1967, 6, 1471-1473.

43. Luu, S. D. N.; Vaqueiro, P. Synthesis, Characterization and Thermoelectric Properties of the Oxytelluride $\mathrm{Bi}_{2} \mathrm{O}_{2}$ Te. J. Sol. State Chem. 2015, 226, 219-223.

44. Merrill, L.; Bassett, W.A. Miniature Diamond Anvil Pressure Cell for Single Crystal $\mathrm{X} \square$ Ray Diffraction Studies. Rev. Sci. Instr. 1974, 45, 290-294. 
45. Mao, H. K.; Xu, J.; Bell, P. M. Calibration of the Ruby Pressure Gauge to $800 \mathrm{Kbar}$ under Quasi-Hydrostatic Conditions. J. Geophys. Res. 1986, 91, 4673 - 4676.

46. Fauth, F.; Peral, I.; Popescu, C.; Knapp, M. The New Material Science Powder Diffraction Beamline at ALBA Synchrotron. Powder Diffr. 2013, 28, S360 - S370.

47. Dewaele, A.; Loubeyre, P.; Mezouar, M. Equations of State of Six Metals Above 94 GPa. Phys. Rev. B 2004, 70, 094112.

48. Prescher, C.; Prakapenka, V. B. DIOPTAS: A Program for Reduction of TwoDimensional X-Ray Diffraction Data and Data Exploration. High Press. Res. 2015, 35 , 223-230.

49. Rodriguez-Carvajal, J. Recent Advances in Magnetic Structure Determination by Neutron Powder Diffraction. Phys. B: Cond.Mat. 1993, 192, 55-69.

50. Nolze, G.; Kraus, W. PowderCell 2.0 for Windows. Powder Diffr. 1998, 13, 256-259.

51. Letoullec, R.; Pinceaux, J. P.; Loubeyre, P. The Membrane Diamond Anvil Cell: A New Device for Generating Continuous Pressure and Temperature Variations. High Press. Res. 1988, 1, 77-90.

52. Goñi, A. R.; Syassen, K. High Pressure Semiconductor Physics I, Chapter 4, Ed. T. Suski and W. Paul, Academic Press 1998.

53. Klotz, S.; Chervin, J.-C.; Munsch, P.; Le Marchand, G. Hydrostatic Limits of 11 Pressure Transmitting Media. J. Phys. D: Appl. Phys. 2009, 42, 075413-1 - 075413-7.

54. Errandonea, D.; Muñoz, A.; Gonzalez-Platas, J. Comment on "High-Pressure X-Ray Diffraction Study of $\mathrm{YBO}_{3} / \mathrm{Eu}^{3+}, \mathrm{GdBO}_{3}$, and $\mathrm{EuBO}_{3}$ : Pressure-Induced Amorphization in $\mathrm{GdBO}_{3}$ ”. J. Appl. Phys. 2014, 115, 043507]. J. Appl. Phys. 2014, $115,216101$. 
55. Hohenberg, P.; Kohn, W. Inhomogeneous Electron Gas. Phys. Rev. 1964, 136, $864-871$.

56. Kresse, G.; Hafner, J. Ab Initio Molecular Dynamics for Liquid Metals. Phys. Rev. B 1993, $47,558-561$.

57. Blöchl, P. E. Projector Augmented-Wave Method. Phys. Rev. B 1994, 50, $17953-17979$.

58. Perdew, J. P.; Ruzsinszky, A.; Csonka, G. I.; Vydrow, O. A.; Scuseria, G. E.; Constantin, L. A.; Zhou, X.; Burke, K. Restoring the Density-Gradient Expansion for Exchange in Solids and Surfaces. Phys. Rev. Lett. 2008, 100, 136406.

59. Monkhorst, H.J.; Pack, J.D. Special Points for Brillouin-zone integrations. Phys. Rev. $B$ 1976, 13, 5188-5192.

60. Van Quang, T.; Lim, H.; Kim, M. Temperature and Carrier-Concentration Dependences of the Thermoelectric Properties of Bismuth Selenide Dioxide Compounds. J. Kor. Phys. Soc. 2012, 61, 1728-1731.

61. Van Quang, T.; Kim, M. Role of O and Se Defects in the Thermoelectric Properties of Bismuth Oxide Selenide. J. Appl. Phys. 2016, 120, 195105-1 - 195105-5.

62. Bradly, C. J.; Cracknell, A. P. The Mathematical Theory of Symmetry in Solids. Clarendon Press. Oxford, 1972.

63. Aroyo, M. I.; Orobengoa, D.; de la Flor, G.; Tasci, E. S.; Perez-Mato, J. M.; Wondratschek, H. Brillouin-zone Database on the Bilbao Crystallographic Server. Acta Cryst. A 2014, 70, $126-137$.

64. Parlinski, K. Computer Code PHONON. See: http://wolf.ifj.edu.pl/phonon.

65. Nielsen, O.H.; Martin, R.M. Quantum-mechanical Theory of Stress and Force. Phys. Rev. B 1985, 32, 3780-3791. 
66. Le Page, Y.; Saxe, P. Symmetry General Least-Squares Extraction of Elastic Data for Strained Materials From ab Initio Calculations of Stress. Phys. Rev. B 2002, 65, 104104.

67. Fujihisa, H.; Akahama, Y.; Kawamura, H.; Ohishi, Y.; Shimomura, O.; Yamawaki, H.; Sakashita, M.; Gotoh, Y.; Takeya, S.; Honda, K. O8 Cluster Structure of the Epsilon Phase of Solid Oxygen. Phys. Rev. Lett. 2006, 97(8), 085503.

68. Lundergaard, L. F.; Weck, G.; McMahon, M. I.; Desgreniers, S.; Loubeyre, P. Observation of an $\mathrm{O}_{8}$ Molecular Lattice in the Epsilon Phase of Solid Oxygen. Nature 2006, 443, 201-204.

69. Birch, F. J. Finite Strain Isotherm and Velocities for Single-Crystal and Polycrystalline $\mathrm{NaCl}$ at High Pressures and 300K. Geophys. Res. 1978, 83, 1257-1268.

70. Sakai, N.; Kajiwara, T.; Takemura, K.; Minomura, S.; Fujii, Y. Pressure-Induced Phase Transition in $\mathrm{Sb}_{2} \mathrm{Te}_{3}$. Sol. State Commun. 1981, 40, 1045-1047.

71. Polian, A.; Gauthier, M.; Souza, S. M.; Triches, D. M.; Cardoso de Lima, J.; Grandi, T. A. Two-Dimensional Pressure-Induced Electronic Topological Transition in $\mathrm{Bi}_{2} \mathrm{Te}_{3}$. Phys. Rev. B 2011, 83, 113106.

72. Kroumova, E.; Aroyo, M. I.; Perez Mato, J. M.; Kirov, A.; Capillas, C.; Ivantchev, S.; Wondratschek, H. Bilbao Crystallographic Server: Useful Databases and Tools for Phase Transitions Studies. Phase Transitions 2003, 76, 155-170.

73. Canepa, P.; Hanson, R. M.; Ugliengo, P.; Alfredsson, M. J-ICE: A New Jmol Interface for Handling and Visualizing Crystallographic and Electronic Properties. J. Appl. Cryst. 2011, 44, 225-229. 
74. Lockwood, D. J.; Yu, G.; Rowell, N. L. Optical Phonon Frequencies and Damping in AlAs, GaP, GaAs, InP, InAs and InSb Studied by Oblique Incidence Infrared Spectroscopy. Sol. State Comm. 2005, 136, 404-409.

75. Ibáñez, J.; Oliva, R.; Manjón, F. J.; Segura, A.; Yamaguchi, T.; Nanishi, Y.; Cusco, R.; Artus, L. High-Pressure Lattice Dynamics in Wurtzite and Rocksalt Indium Nitride Investigated by Means of Raman Spectroscopy. Phys. Rev. B 2013, 88, 115202.

76. Hauber, A.; Fahy, S. Scattering of Carriers by Coupled Plasmon-Phonon Modes in Bulk Polar Semiconductors and Polar Semiconductor Heterostructures. Phys. Rev. B 2017, 95, 045210.

77. Pereira, A. L. J.; Gomis, O.; Sans, J. A.; Contreras-García, J.; Manjón, F. J.; Rodríguez-Hernández, P.; Muñoz, A.; Beltrán, A. $\beta-\mathrm{Bi}_{2} \mathrm{O}_{3}$ Under Compression: Optical and Elastic Properties and Electron Density Topology Analysis. Phys. Rev. B 2016, 93, 224111-1-224111-13.

78. Akahama, Y.; Kawamura, H. High-Pressure Raman Spectroscopy of Solid Oxygen. Phys. Rev. B 1996, 54, R15602 - R15605.

79. Halsall, M. P.; Harmer, P.; Parbrook, P. J.; Henley, S. J. Raman Scattering and Absorption Study of the High-Pressure Wurtzite to Rocksalt Phase Transition of GaN. Phys. Rev. B 2004, 69, 235207.

80. Manjón, F. J.; Errandonea, D.; Romero, A. H.; Garro, N.; Serrano, J.; Kuball, M. Lattice Dynamics of Wurtzite and Rocksalt AlN Under High Pressure: Effect of Compression on the Crystal Anisotropy of Wurtzite-Type Semiconductors. Phys. Rev. B 2008, 77, 205204. 
81. Manjón, F. J.; Syassen, K.; Lauck, R. Effect of Pressure on Phonon Modes in Wurtzite Zinc Oxide. High Pres. Res. 2002, 22, 299-304.

82. Wang, Y. Q.; Lu, P. C.; Wu, J. J.; Liu, J.; Wang, X. C.; Zhao, J. Y.; Bi, W.; Alp, E. E.; Park, C. Y.; Popov, D.; et al. Phonon Density of States of Single-Crystal $\mathrm{SrFe}_{2} \mathrm{As}_{2}$ across the Collapse Phase Transition at High Pressure. Phys. Rev. B 2016, 94, 014516.

83. Urbach, F. The Long-Wavelength Edge of Photographic Sensitivity and of the Electronic Absorption of Solids. Phys. Rev. 1953, 92, 1324.

84. Tauc, J. Optical Properties and Electronic Structure of Amorphous Ge and Si. Mater. Res. Bull. 1968, 3, 37- 46.

85. Errandonea, D.; Muñoz, A.; Rodríguez-Hernández, P.; Proctor, J. E.; Sapiña, F.; Bettinelli, M. Theoretical and Experimental Study of the Crystal Structures, Lattice Vibrations, and Band Structures of Monazite-Type $\mathrm{PbCrO}_{4}, \mathrm{PbSeO}_{4}, \mathrm{SrCrO}_{4}$, and $\mathrm{SrSeO}_{4}$. Inorg. Chem., 2015, 54 (15), 7524-7535.

86. Ruiz-Fuertes, J.; Errandonea, D.; Manjón, F. J.; Martínez-García, D.; Segura, A.; Ursaki, V. V.; Tiginyanu, I. M. High-Pressure Effects on the Optical-Absorption Edge of $\mathrm{CdIn}_{2} \mathrm{~S}_{4} \mathrm{CdIn}_{2} \mathrm{~S}_{4}, \mathrm{MgIn}_{2} \mathrm{~S}_{4} \mathrm{MgIn}_{2} \mathrm{~S}_{4}$, and $\mathrm{MnIn}_{2} \mathrm{~S}_{4} \mathrm{MnIn}_{2} \mathrm{~S}_{4}$ Thiospinels. J. Appl. Phys. 2008, 103, 063710.

87. Santamaria-Perez, D.; Ruiz-Fuertes, J. $A B_{2} S_{4}$ and $A B_{2} S_{4}$ Compounds at High Pressures. in Pressure-Induced Phase Transitions in $A B_{2} X_{4}$ Chalcogenide Compounds. Springer Verlag., 75-102, 2014.

88. Manjón, F. J.; Gomis, O.; Rodríguez-Hernández, P.; Pérez-González, E.; Muñoz, A.; Errandonea, D.; Ruiz-Fuertes, J.; Segura, A.; Fuentes-Cabrera, M.; Tiginyanu, I. M.; et al. Nonlinear Pressure Dependence of the Direct Band Gap in Adamantine OrderedVacancy Compounds. Phys. Rev. B 2010, 81, 195201. 
89. Errandonea, D.; Muñoz, A.; Rodríguez-Hernández, P.; Gomis, O.; Achary, S. N.; Popescu, C.; Patwe, S. J.; Tyagi, A. K. High-Pressure Crystal Structure, Lattice Vibrations, and Band Structure of $\mathrm{BiSbO}_{4}$. Inorg. Chem. 2016, 55 (10), 4958-4969. 


\section{Tables}

Table 1 - EOS parameters and axial compressibilities of $\mathrm{Bi}_{2} \mathrm{O}_{2} \mathrm{Se}$ at room pressure.

\begin{tabular}{cccccc}
\hline & $\begin{array}{c}\mathrm{V}_{0} \\
\left(\AA^{3}\right)\end{array}$ & $\begin{array}{c}\mathrm{B}_{0} \\
(\mathrm{GPa})\end{array}$ & $B_{0}^{s}$ & $\begin{array}{c}\kappa_{\mathrm{a}} \\
\left(10^{-3} \mathrm{GPa}^{-1}\right)\end{array}$ & $\begin{array}{c}\kappa_{\mathrm{c}} \\
\left(10^{-3} \mathrm{GPa}^{-1}\right)\end{array}$ \\
\hline Experimental & $184.69(8)$ & $71.5(13)$ & $5.6(3)$ & $3.6(2)$ & $6.4(2)$ \\
Theoretical & 183.8 & 88.17 & 4.86 & 3.0 & 5.9 \\
\hline
\end{tabular}

Table 2 - Experimental and theoretical Raman (R) and infrared (IR) mode frequencies of $\mathrm{Bi}_{2} \mathrm{O}_{2} \mathrm{Se}$ at room pressure.

\begin{tabular}{|c|c|c|c|}
\hline Symmetry & $\begin{array}{l}\text { Exp. Freq. } \\
\left(\mathrm{cm}^{-1}\right)\end{array}$ & $\begin{array}{c}\text { Theor. Freq. } \\
\left(\mathrm{cm}^{-1}\right)\end{array}$ & Assignment \\
\hline$E_{u}(I R)$ & & 59.2 & $\begin{array}{c}\text { Bi and Se out-of-phase movement } \\
\text { along the layer (shear interlayer } \\
\text { mode) }\end{array}$ \\
\hline$A_{2 u}(I R)$ & & 64.5 & $\begin{array}{l}\text { Bi and Se out of-phase movement } \\
\text { perpendicular to the layer } \\
\text { (longitudinal interlayer mode) }\end{array}$ \\
\hline$E_{g}(R)$ & & 72.0 & $\begin{array}{l}\text { Bi out-of-phase interlayer movement } \\
\text { along the layer }\end{array}$ \\
\hline $\mathbf{A}_{1 \mathrm{~g}}(\mathbf{R})$ & 159.2 & 165.7 & $\begin{array}{l}\text { Bi out-phase interlayer } \\
\text { movement perpendicular to the } \\
\text { layers (breathing mode of the layers) }\end{array}$ \\
\hline$E_{u}(I R)$ & & 293.9 & $\begin{array}{c}\mathrm{O} \text { in-phase intralayer movement } \\
\text { along the layer }\end{array}$ \\
\hline $\mathbf{B}_{\mathrm{g}}(\mathbf{R})$ & & 369.4 & $\begin{array}{c}\text { O out-of-phase intralayer movement } \\
\text { perpendicular to the layer }\end{array}$ \\
\hline$A_{2 u}(I R)$ & & 402.8 & $\begin{array}{l}\text { O in-phase intralayer movement } \\
\text { perpendicular to the layer }\end{array}$ \\
\hline$E_{g}(R)$ & & 444.0 & $\begin{array}{c}\text { O out-of-phase intralayer movement } \\
\text { along the layer }\end{array}$ \\
\hline
\end{tabular}


Table 3 - Experimental and theoretical first-order Raman (R) and infrared (IR) vibrational mode frequencies and pressure coefficients at room pressure in $\mathrm{Bi}_{2} \mathrm{O}_{2} \mathrm{Se}$ as obtained by a fit with the equation $\omega(\mathrm{P})=\omega_{0}+a \cdot \mathrm{P}$ in the pressure range from 0 to $4 \mathrm{GPa}$. The frequencies and pressure coefficients of other modes observed at different pressures (indicated) are also provided.

\begin{tabular}{|c|c|c|c|c|}
\hline \multirow[b]{2}{*}{ Symmetry } & \multicolumn{2}{|c|}{ Experimental } & \multicolumn{2}{|c|}{ Theoretical } \\
\hline & $\begin{array}{c}\omega_{0} \\
\left(\mathrm{~cm}^{-1}\right)\end{array}$ & $\begin{array}{c}a \\
\left(\mathrm{~cm}^{-1} / \mathrm{GPa}\right)\end{array}$ & $\begin{array}{c}\omega_{0} \\
\left(\mathrm{~cm}^{-1}\right)\end{array}$ & $\begin{array}{c}a \\
\left(\mathrm{~cm}^{-1} / \mathrm{GPa}\right)\end{array}$ \\
\hline $\mathbf{E}_{\mathrm{u}}(\mathrm{IR})$ & & & $59.2(3)$ & $5.5(2)$ \\
\hline $\mathbf{A}_{2 u}(\mathrm{IR})$ & & & $64.5(1)$ & $4.1(9)$ \\
\hline $\mathbf{E}_{\mathrm{g}}(\mathbf{R})$ & $59.5(5)$ & $1.0(2)$ & $72.0(1)$ & $1.1(8)$ \\
\hline$A_{1 g}(R)$ & $160.5(6)$ & $2.1(3)$ & $165.7(3)$ & $2.1(2)$ \\
\hline$E_{u}(I R)$ & & & $293.9(1)$ & $4.2(6)$ \\
\hline$\overline{B_{g}(R)}$ & & & $369.4(5)$ & $4.0(5)$ \\
\hline $\mathbf{A}_{2 u}(\mathrm{IR})$ & & & $402.8(6)$ & $3.1(4)$ \\
\hline$E_{g}(R)$ & & & $444.0(1)$ & $5.3(7)$ \\
\hline P1 (6.6 GPa) & $137(3)$ & $1.9(4)$ & & \\
\hline P2 (10.0 GPa) & $280(8)$ & $1.1(5)$ & & \\
\hline P3 (10.0 GPa) & $285(7)$ & $2.6(4)$ & & \\
\hline P4 (6.6 GPa) & $296(4)$ & $4.0(2)$ & & \\
\hline P5 (10.0 GPa) & $381(7)$ & $1.8(3)$ & & \\
\hline P6 (10.0 GPa) & $447(5)$ & $2.5(2)$ & & \\
\hline P7 (10.0 GPa) & $537(8)$ & $0.4(4)$ & & \\
\hline P8 (10.0 GPa) & $568(6)$ & $1.2(2)$ & & \\
\hline
\end{tabular}




\section{Figure Captions}

Figure 1. (color online) (top) Crystalline structure of tetragonal $\mathrm{Bi}_{2} \mathrm{O}_{2} \mathrm{Se}$ at 1 atm. Large magenta balls represent $\mathrm{Bi}$ atoms, while small red balls represent $\mathrm{O}$ atoms and mediumsized green balls represent $\mathrm{Se}$ atoms. Bi atoms are linked to four $\mathrm{O}$ atoms with short bonds (solid lines) and to four Se atoms with long bonds (dashed lines). $\mathrm{O}$ atoms are surrounded by four $\mathrm{Bi}$ atoms and $\mathrm{Se}$ atoms are surrounded by eight $\mathrm{Bi}$ atoms. The structure is composed by alternate $\mathrm{Bi}_{2} \mathrm{O}_{2}$ layers, where $\mathrm{Bi}$ atoms show fourfold coordination, and atomic Se layers. (bottom) Brillouin zone of the body-centered tetragonal unit cell with c/a $>1$.

Figure 2. (color online) Angle-dispersive XRD patterns of $\mathrm{Bi}_{2} \mathrm{O}_{2} \mathrm{Se}$ measured at different pressures up to $22 \mathrm{GPa}$ at room temperature and on decreasing pressure to room pressure (top pattern). The low-pressure patterns (in circles) include the calculated LeBail (black line) and difference (red line) XRD profiles. Blue and black vertical markers indicate the Bragg reflections of the tetragonal $\mathrm{Bi}_{2} \mathrm{O}_{2} \mathrm{Se}$ phase and the $\mathrm{Cu}$ pressure gauge, respectively.

Figure 3. (color online) Experimental (symbols) and theoretical (lines) pressure dependence of the lattice parameters $a$ and $c$ (left) and of the unit-cell volume (right) for $\mathrm{Bi}_{2} \mathrm{O}_{2} \mathrm{Se}$. Insets show the pressure dependence of the $\mathrm{z}$ coordinate of $\mathrm{Bi}$ atoms (left) and the $c / a$ ratio (right). Experimental data include those from our in-home Xcalibur diffractometer (squares) and from the ALBA-CELLS beamline (circles). Errors in the determination of parameters are indicated by symbol sizes. In the volume plot, the solid black line is the EOS fit to the theoretical data while the dashed red line is the EOS fit to experimental data in the quasi-hydrostatic pressure range.

Figure 4. (color online) Pressure dependence of the theoretical interatomic and interplanar distances in $\mathrm{Bi}_{2} \mathrm{O}_{2} \mathrm{Se}$.

Figure 5. (color online) (left) Room-temperature Raman spectra of $\mathrm{Bi}_{2} \mathrm{O}_{2} \mathrm{Se}$ at selected pressures up to $28.3 \mathrm{GPa}$. The top spectrum corresponds to the recovered sample after decompression. (right) Experimental (symbols) and theoretical (lines) pressure dependence 
of the Raman-active modes of $\mathrm{Bi}_{2} \mathrm{O}_{2} \mathrm{Se}$. Different colors represent Raman-active modes of different symmetries.

Figure 6. Theoretical PDCs of $\mathrm{Bi}_{2} \mathrm{O}_{2} \mathrm{Se}$ at $0 \mathrm{GPa}$ (top), $12 \mathrm{GPa}$ (center), and 23.7 GPa (bottom) following the path: $X(0,0,1 / 2)-\Gamma(0,0,0)-Z(1 / 2,1 / 2,-1 / 2)-P(1 / 4 ; 1 / 4,1 / 4)-N(0,1 / 2,0)$.

Figure 7. Pressure dependence of generalized stability criteria of $\mathrm{Bi}_{2} \mathrm{O}_{2} \mathrm{Se}$ up to $45 \mathrm{GPa}$.

Figure 8. (color online) Optical absorption spectrum (black) of $\mathrm{Bi}_{2} \mathrm{O}_{2} \mathrm{Se}$ at ambient pressure fitted to a direct-type Urbach law (red). The inset shows the linear range presented by the absorption edge of $\mathrm{Bi}_{2} \mathrm{O}_{2} \mathrm{Se}$ considering either an indirect $\left(\alpha^{1 / 2}\right)$ or a direct-type Urbach tail $(\ln (\alpha))$. The points are experimental data while the straight red lines are guides for the eye.

Figure 9. (color online) Electronic band structure of $\mathrm{Bi}_{2} \mathrm{O}_{2} \mathrm{Se}$ at (top) $0 \mathrm{GPa}$ and $4.8 \mathrm{GPa}$ (bottom) following the path: $X(0,0,1 / 2)-\Gamma(0,0,0)-Z(1 / 2,1 / 2,-1 / 2)-P(1 / 4 ; 1 / 4,1 / 4)-N(0,1 / 2,0)$. Topmost valence bands (in red) and second topmost valence bands (in blue) are colored in order to better show the crossing and anticrossing taking place in these bands with increasing pressure around $4 \mathrm{GPa}$.

Figure 10. (color online) Optical absorption edge of $\mathrm{Bi}_{2} \mathrm{O}_{2} \mathrm{Se}$ on increasing pressure up to $20 \mathrm{GPa}$.

Figure 11. (Color online) (top) Pressure dependence of the band gap of $\mathrm{Bi}_{2} \mathrm{O}_{2} \mathrm{Se}$ obtained from fitting the absorption edge to the Urbach's law. Dots represent the experimental data and the continuous lines are the calculated dependences for the $\Gamma \rightarrow \Gamma$ (red line) and the $\mathrm{Z}$ $\rightarrow \mathrm{Z}$ (blue line) direct transitions after adding $+0.34 \mathrm{eV}$ to compare to the experimentally observed bandgap at ambient pressure. The theoretical $\mathrm{N}-\Gamma$ indirect bandgap of $\mathrm{Bi}_{2} \mathrm{O}_{2} \mathrm{Se}$ is also shown for comparison (pink line). (bottom) Pressure dependence of the Urbach energy in $\mathrm{Bi}_{2} \mathrm{O}_{2} \mathrm{Se}$. 
Figure 12. Pressure evolution of the Se orbitals contribution in the top valence bands at (left) $\Gamma$ and (right) $\mathrm{N}$ points. The sudden change of character of topmost valence bands at $\Gamma$ is indicative of a band crossing while the progressive change of character of topmost valence bands at $\mathrm{N}$ is indicative of a band anticrossing. 
Figure 1(top)

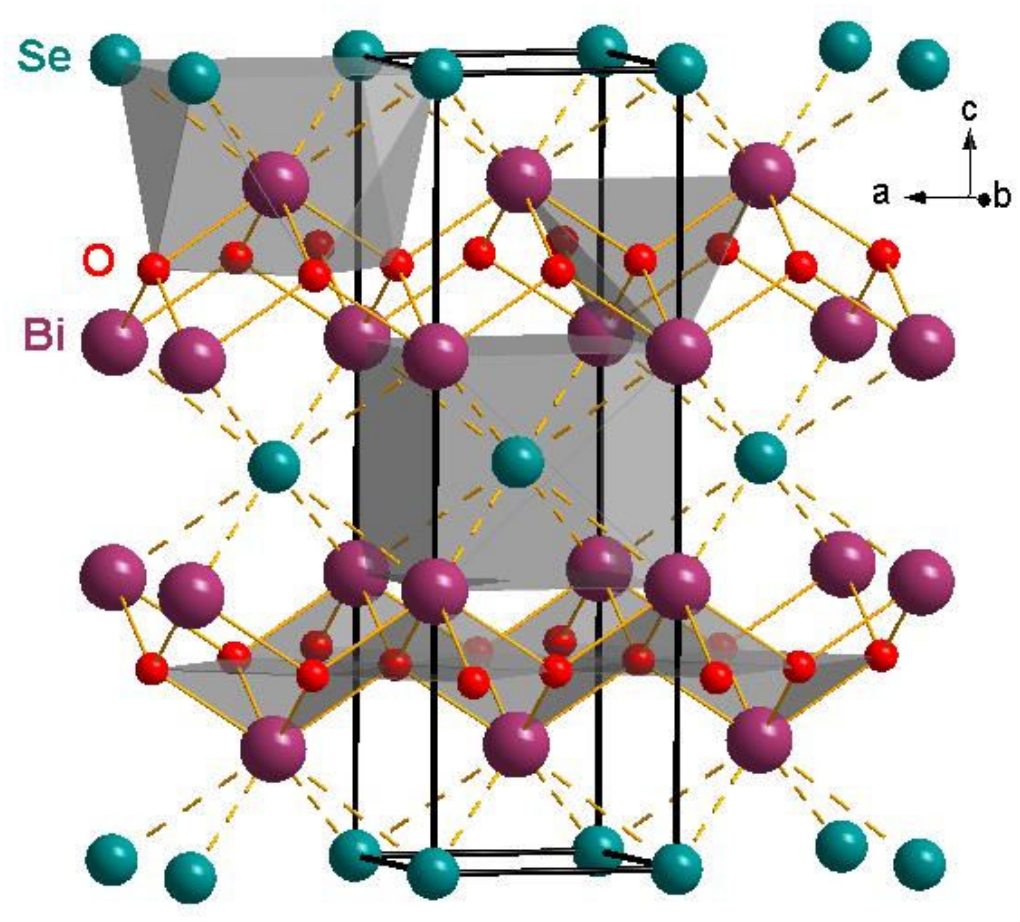


Figure 1(bottom)

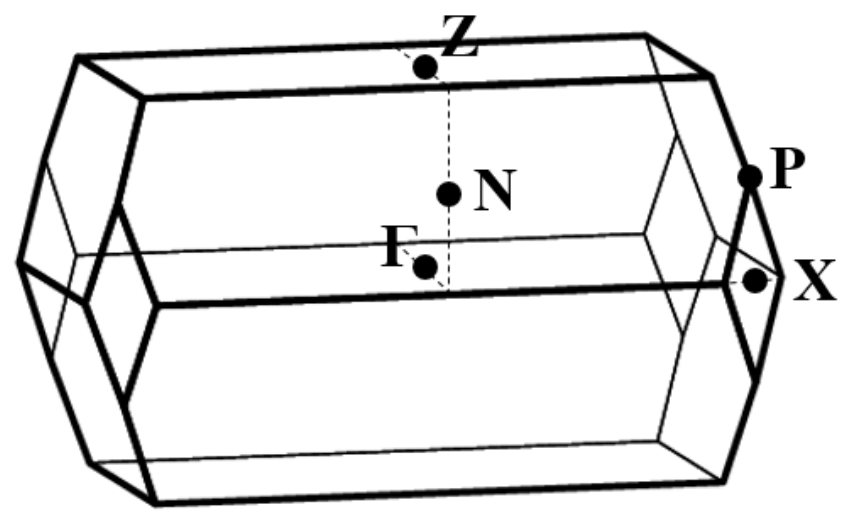


Figure 2

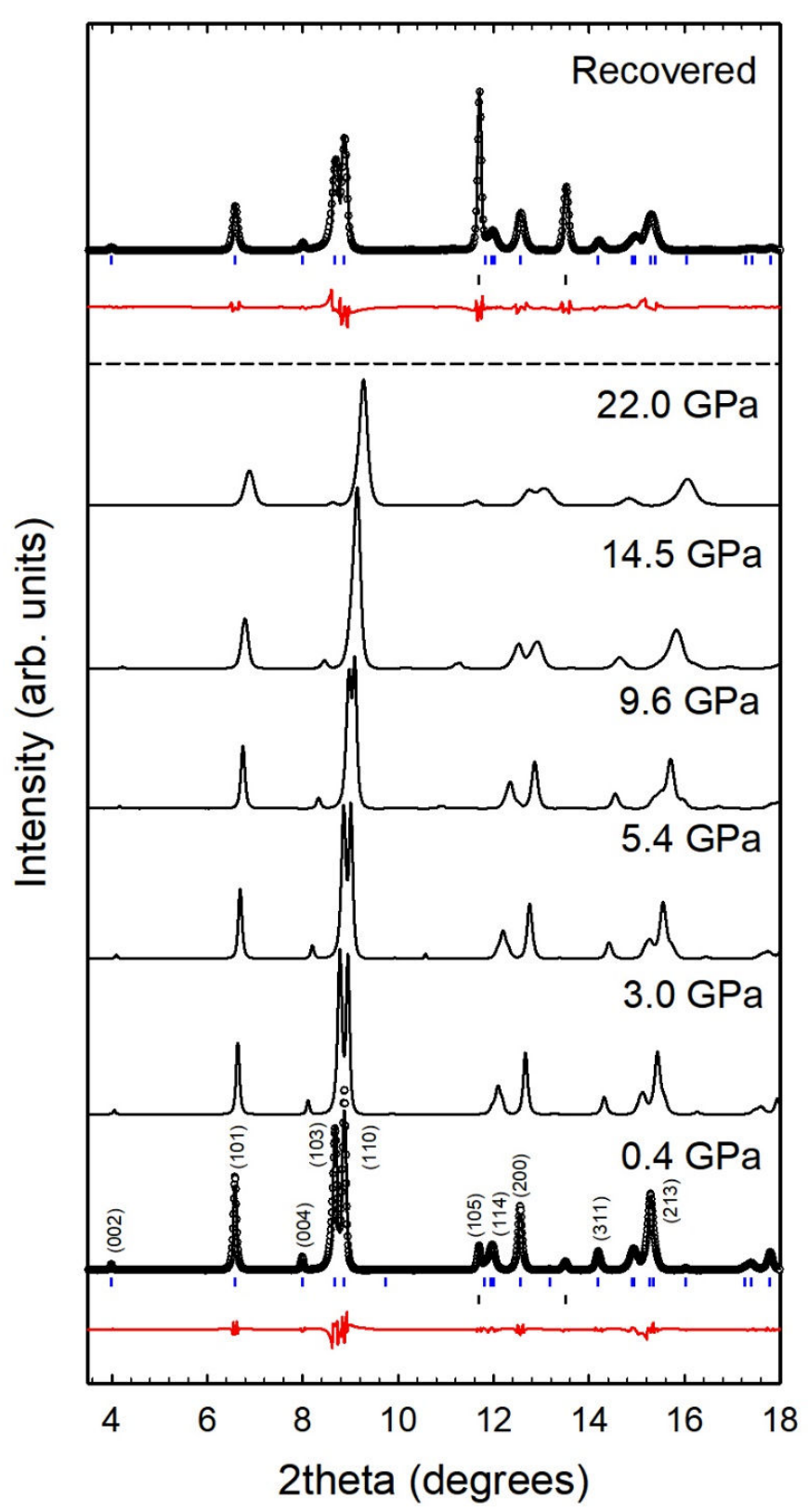


Figure 3(left)

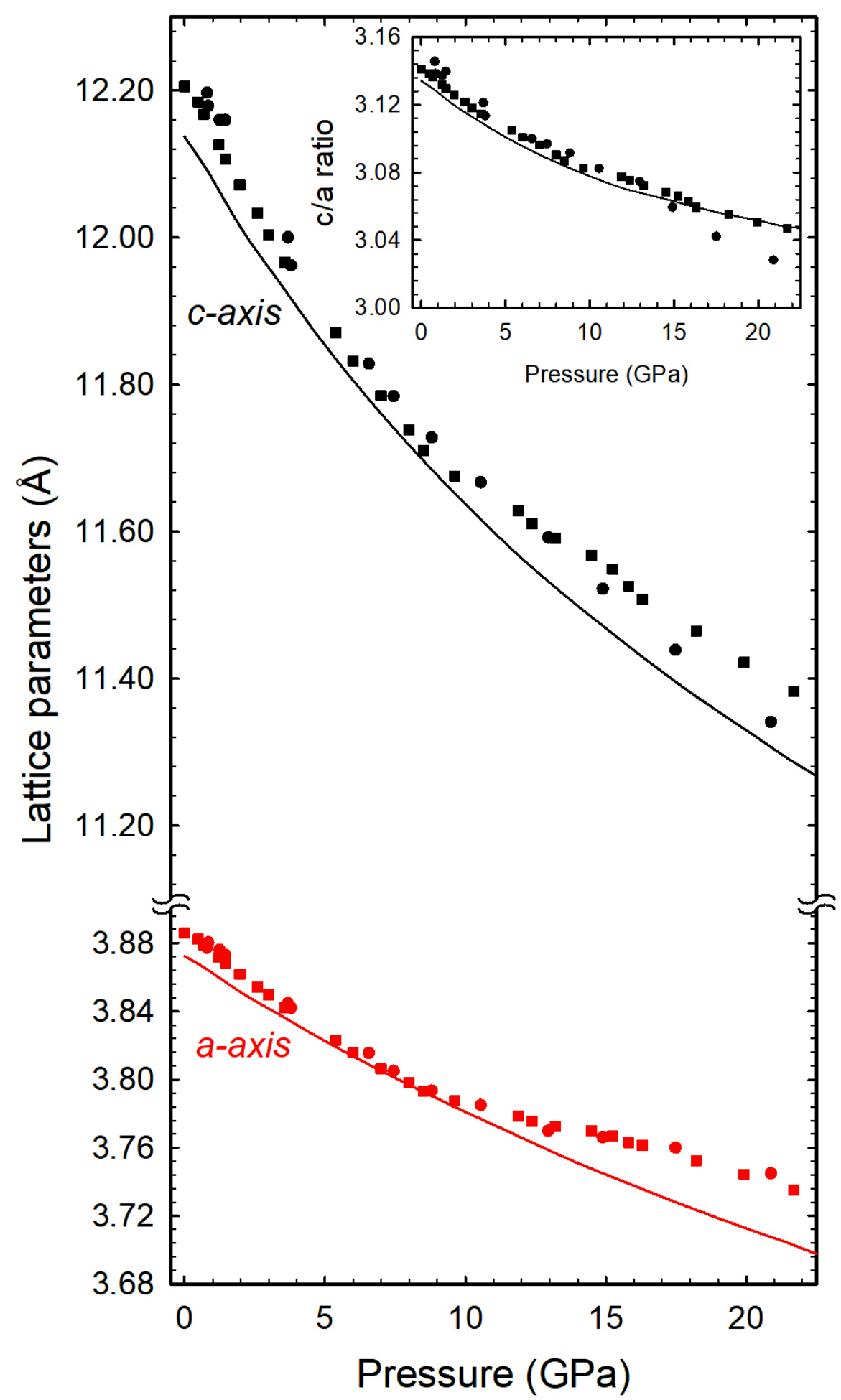


Figure 3(right)

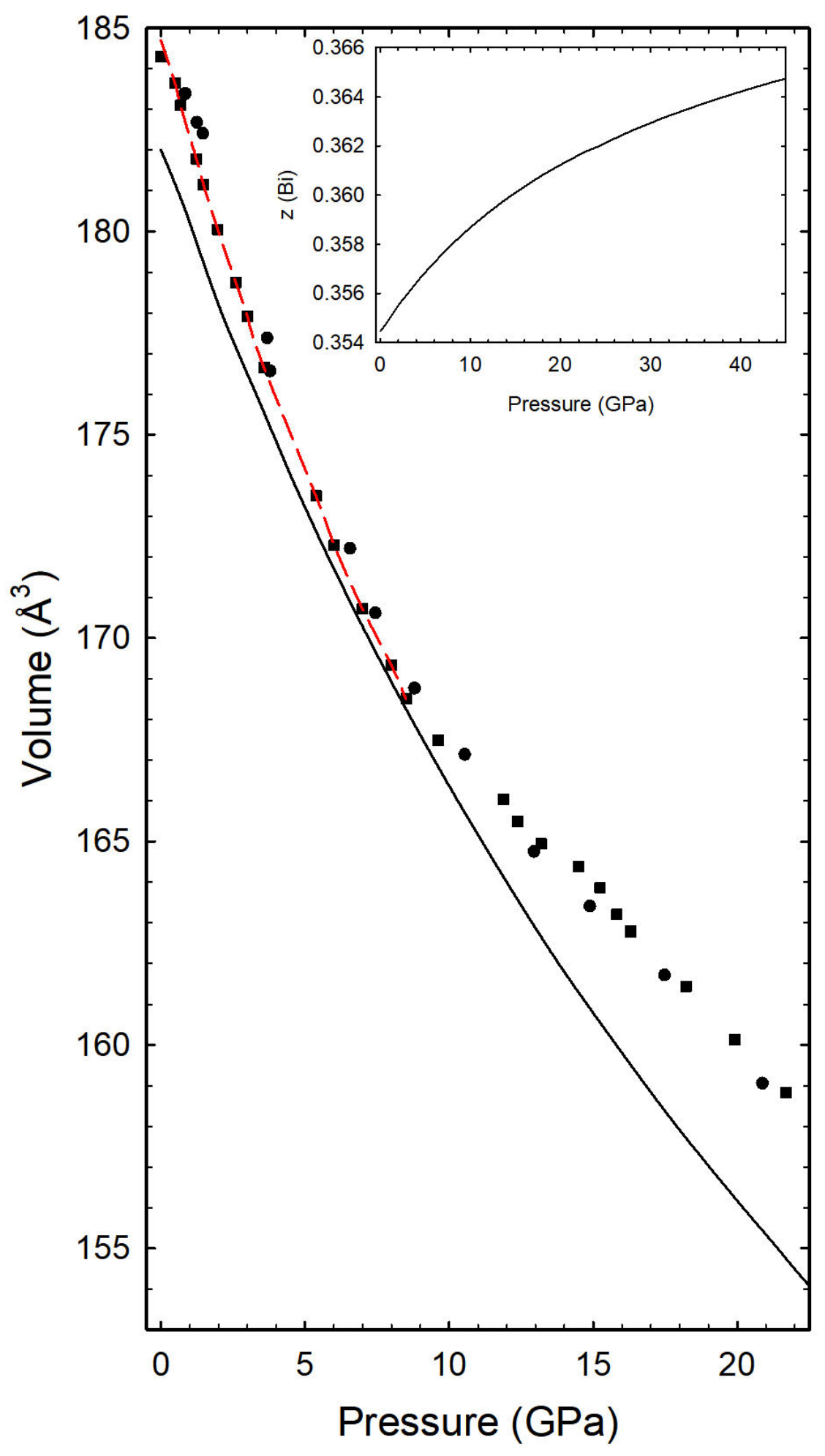


Figure 4

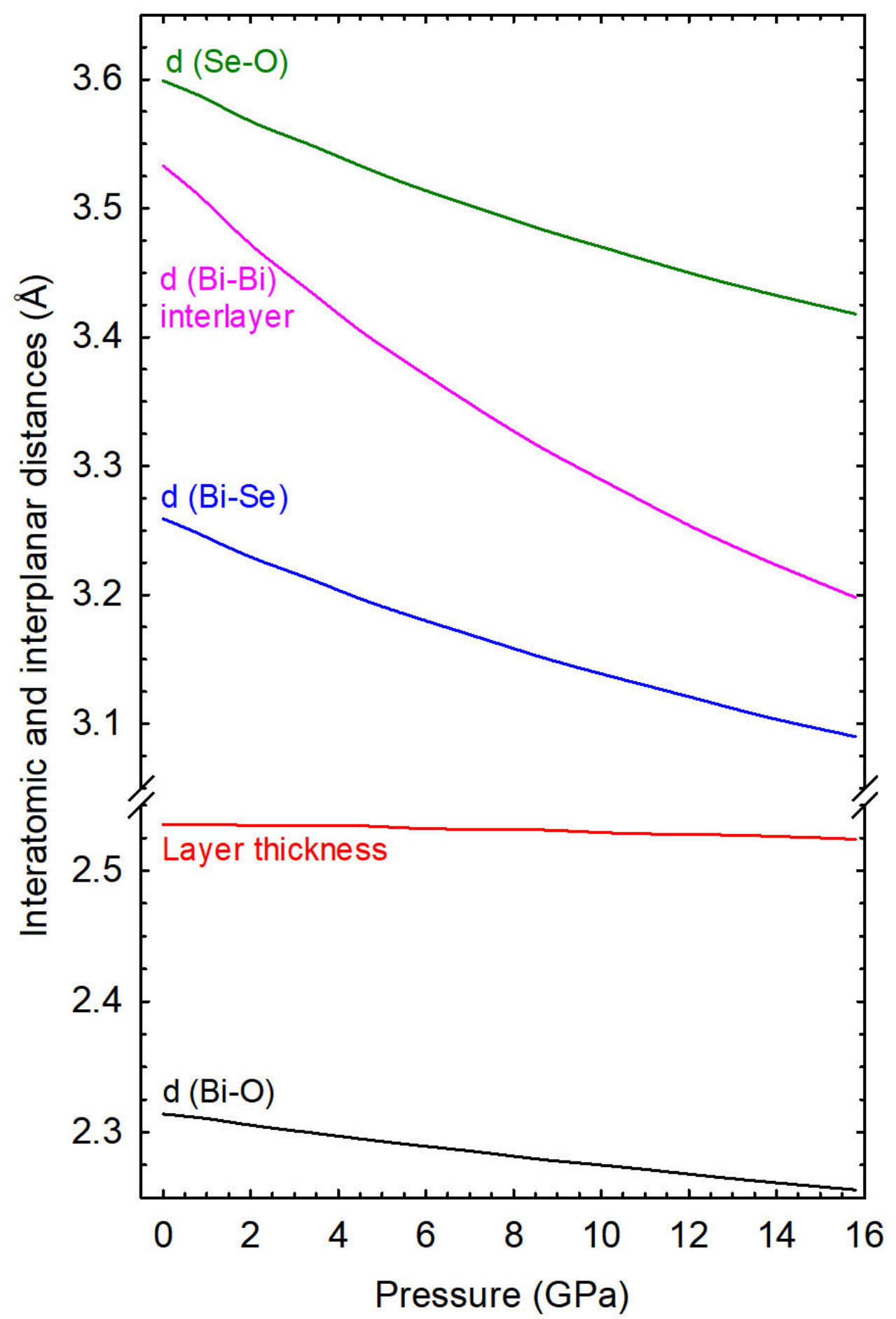


Figure 5 (left)

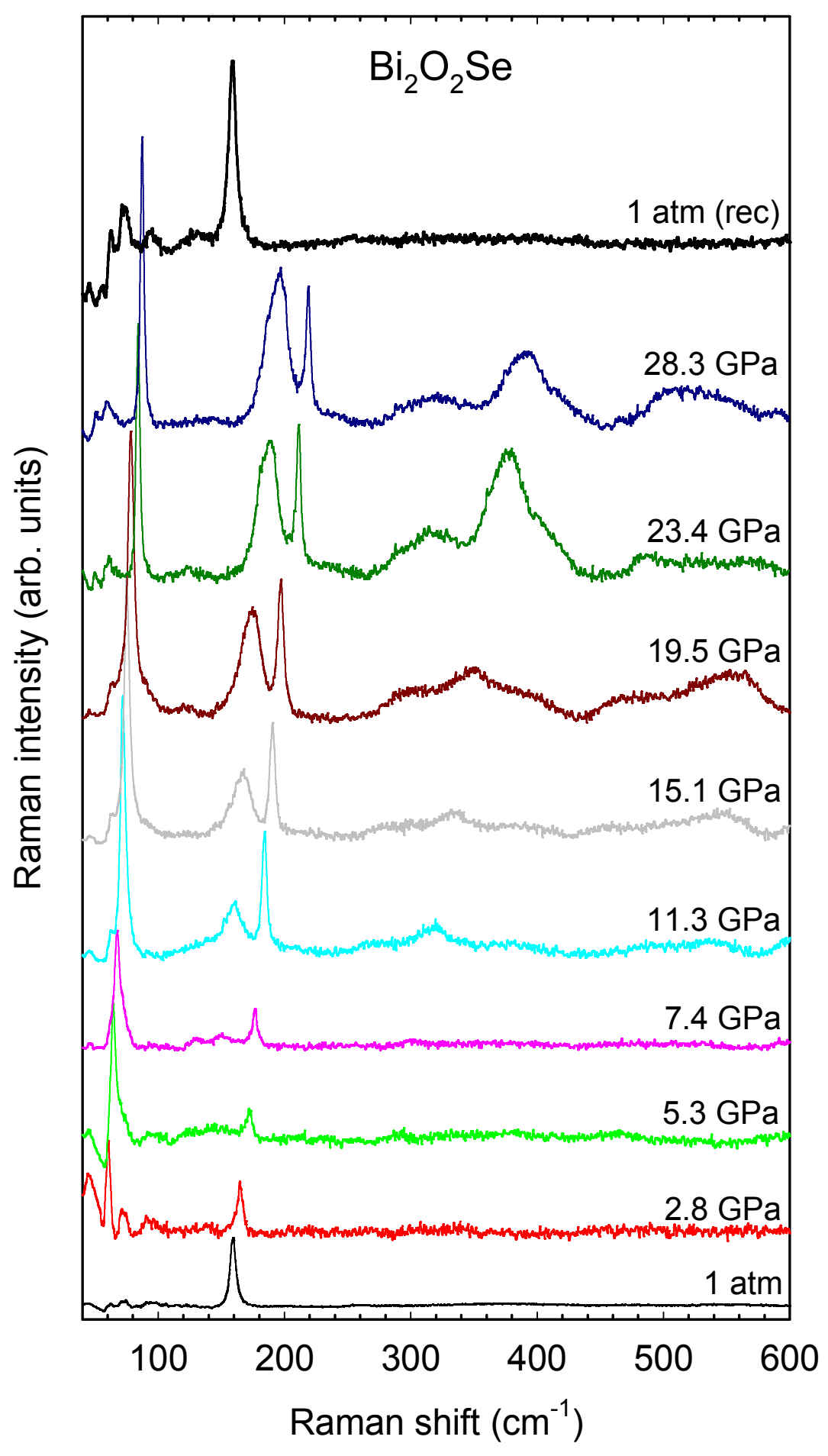


Figure 5 (right)

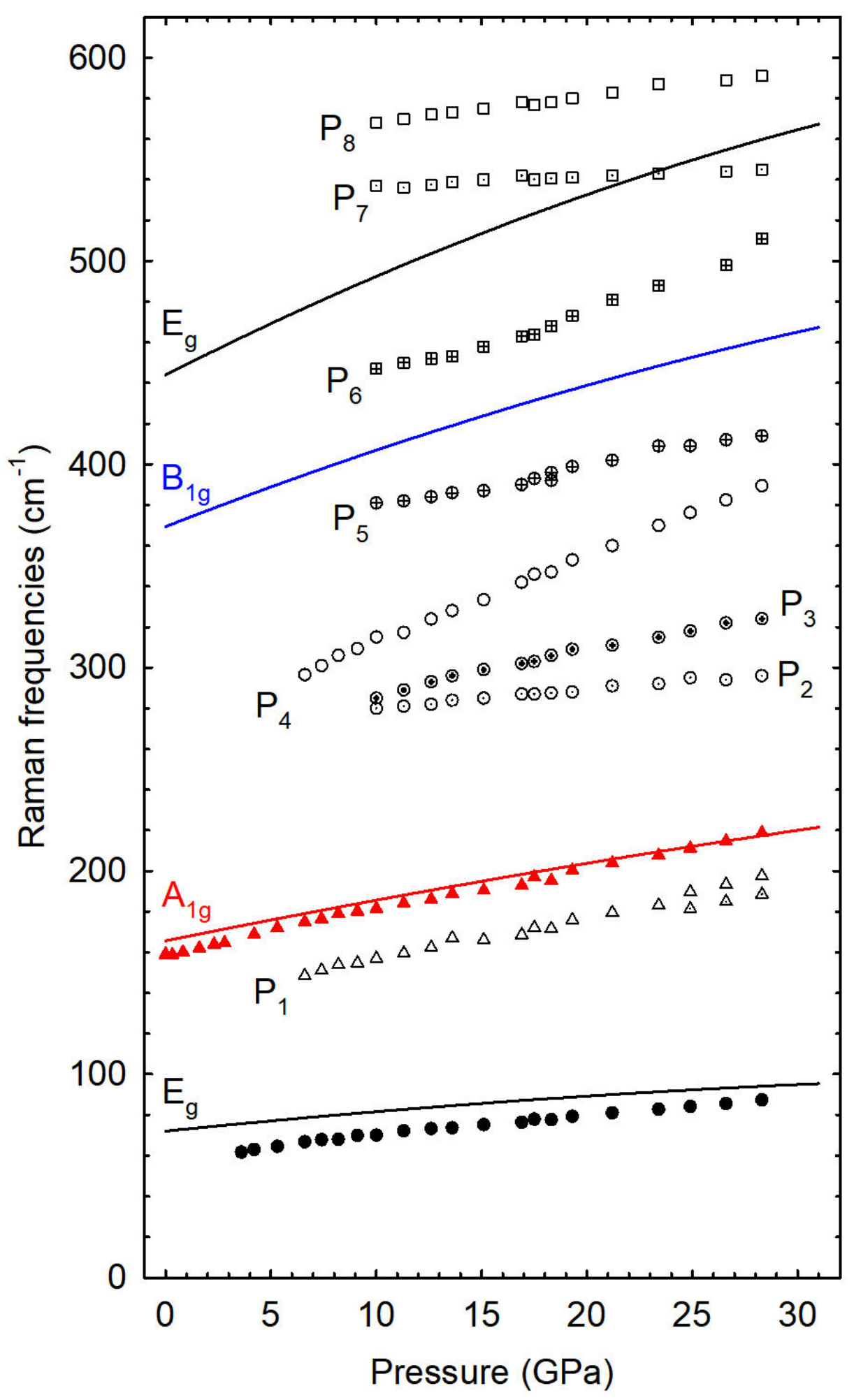


Figure 6
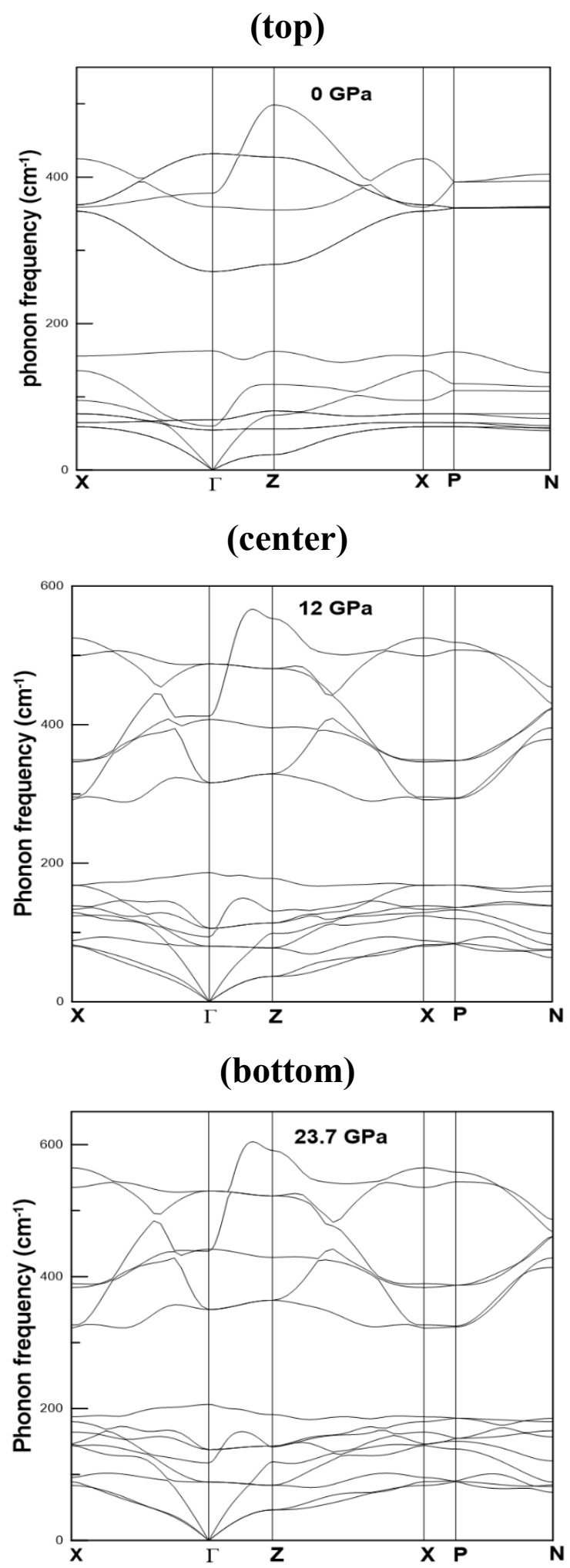
Figure 7

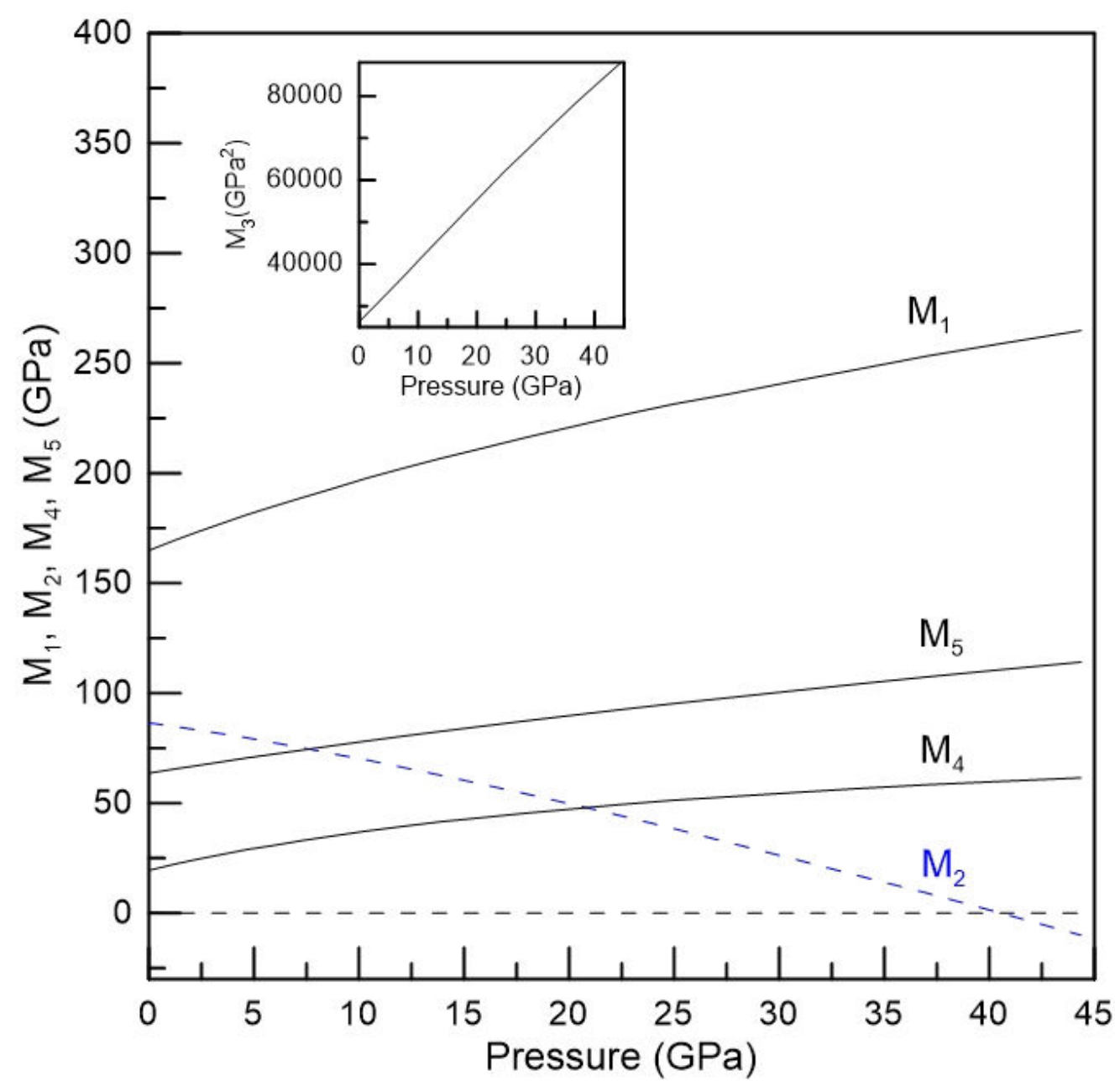


Figure 8

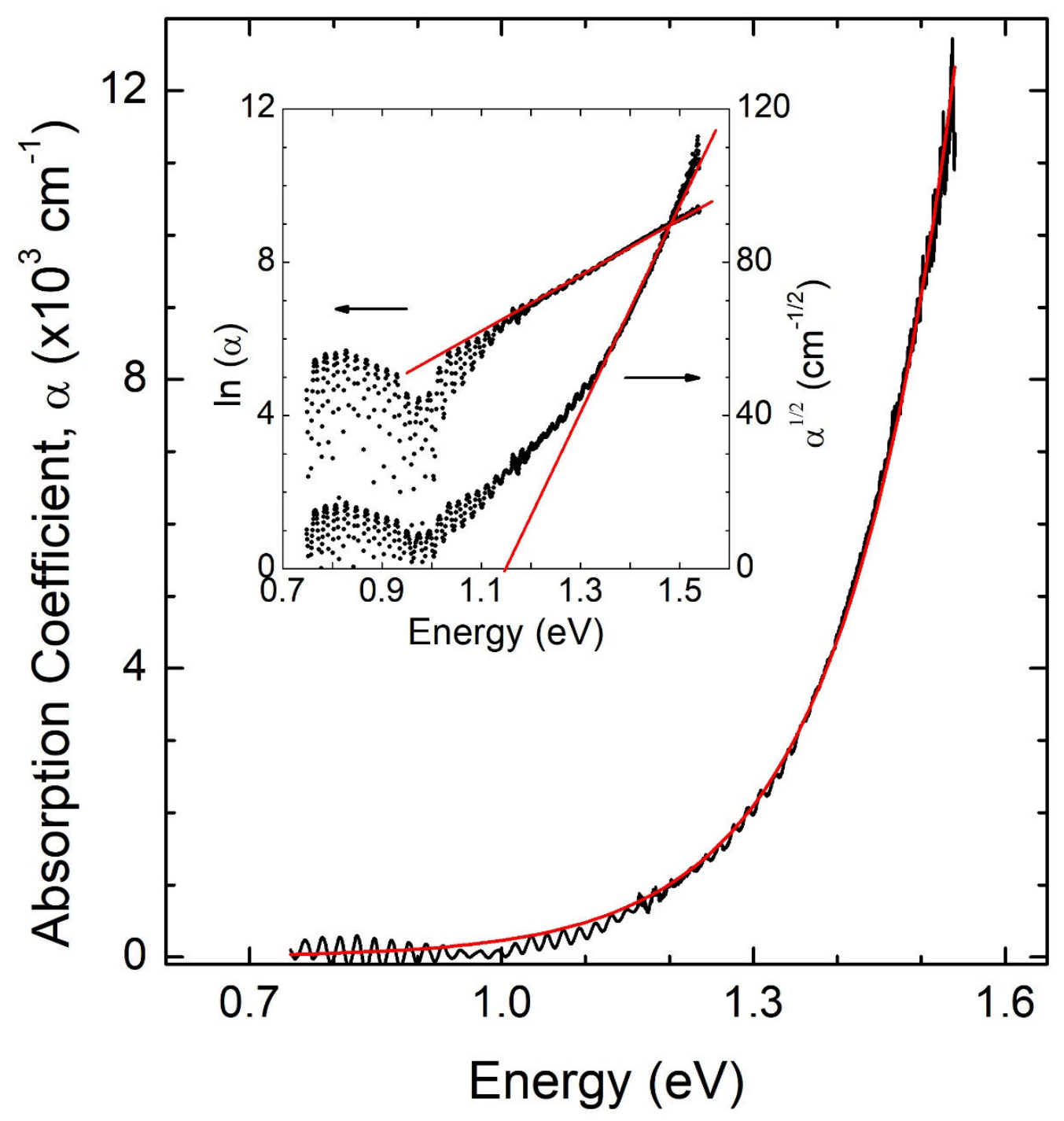


Figure 9

(top)
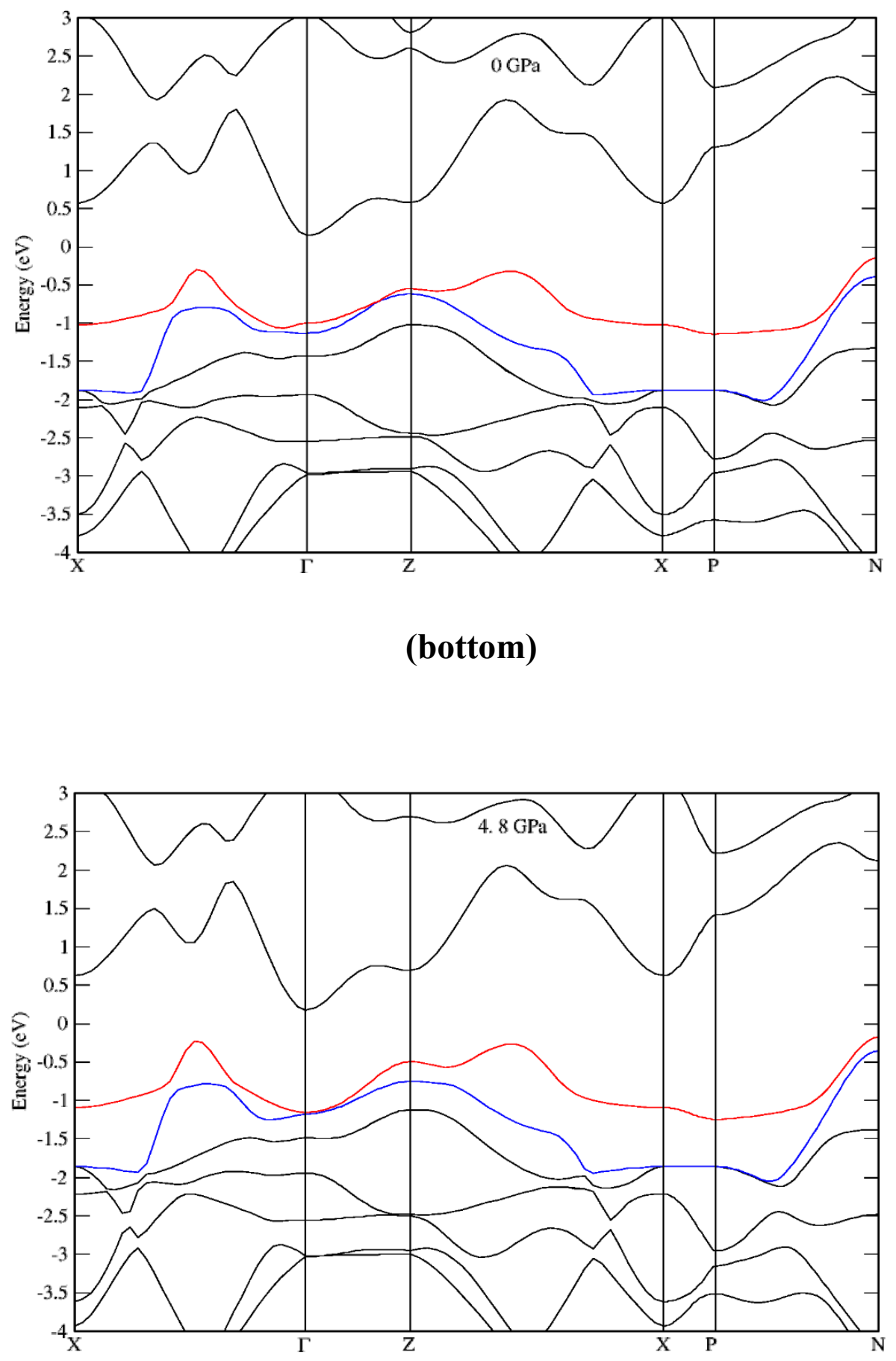
Figure 10

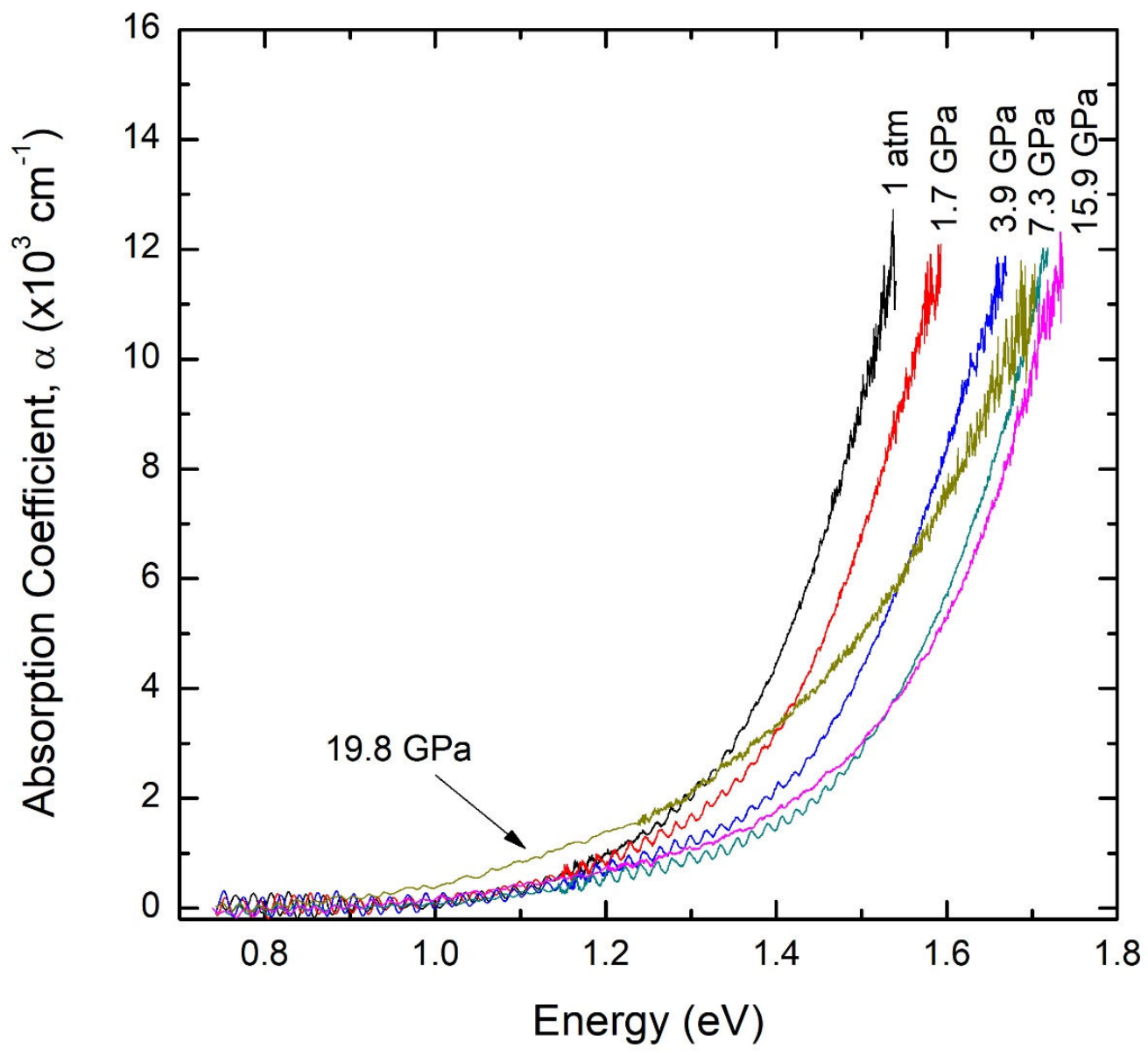


Figure 11
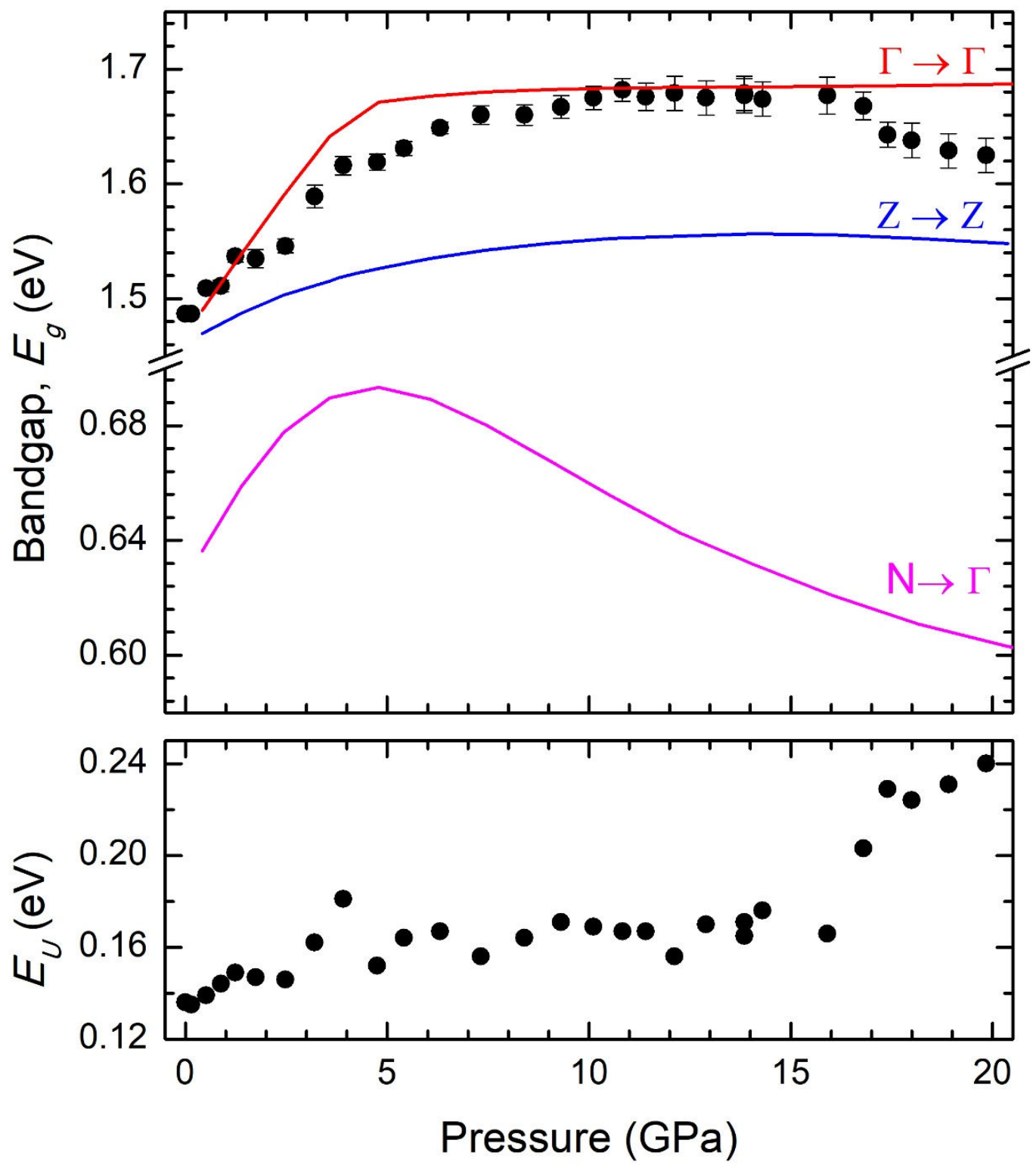
Figure 12 (left)

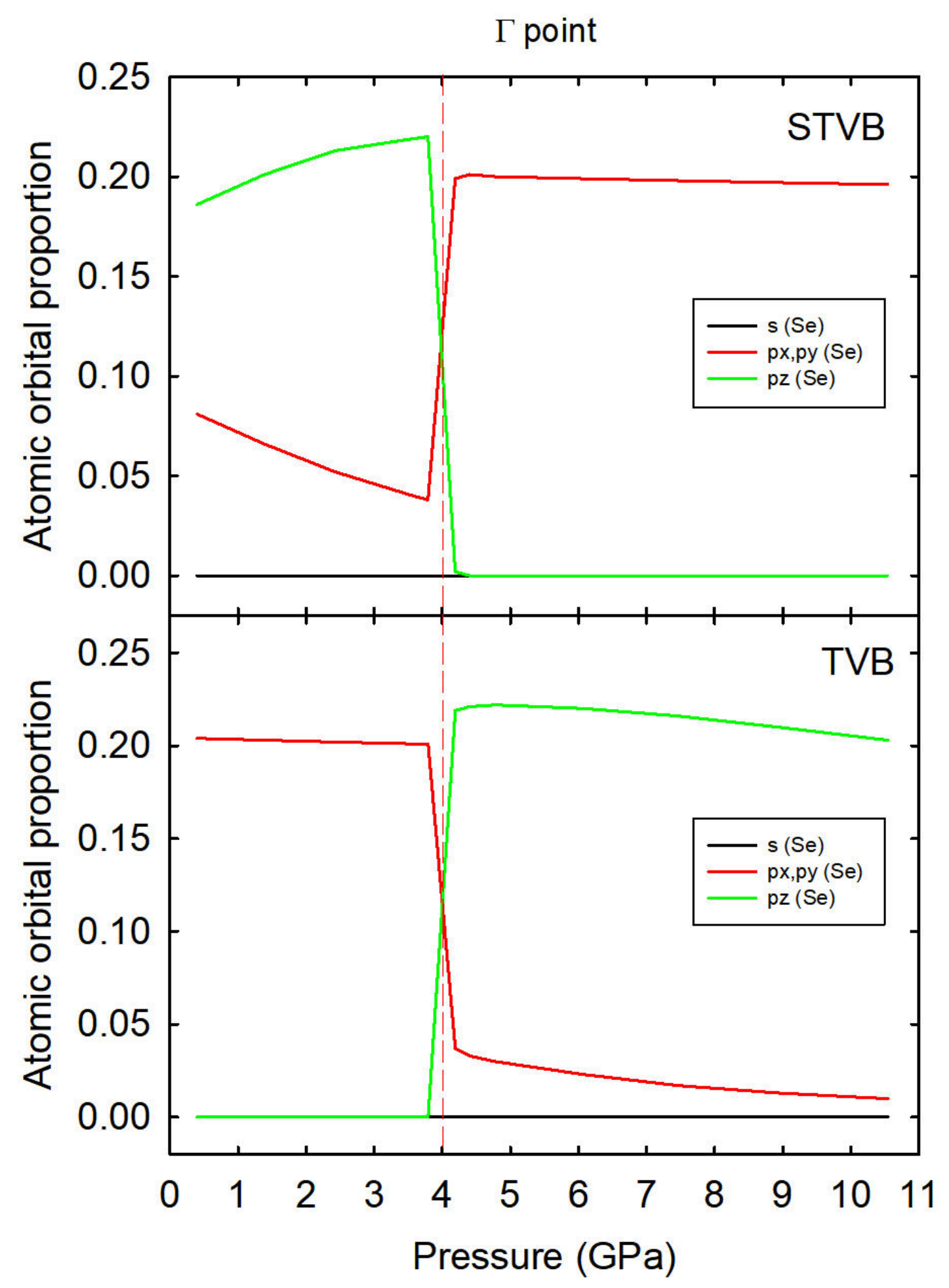


Figure 12(right)

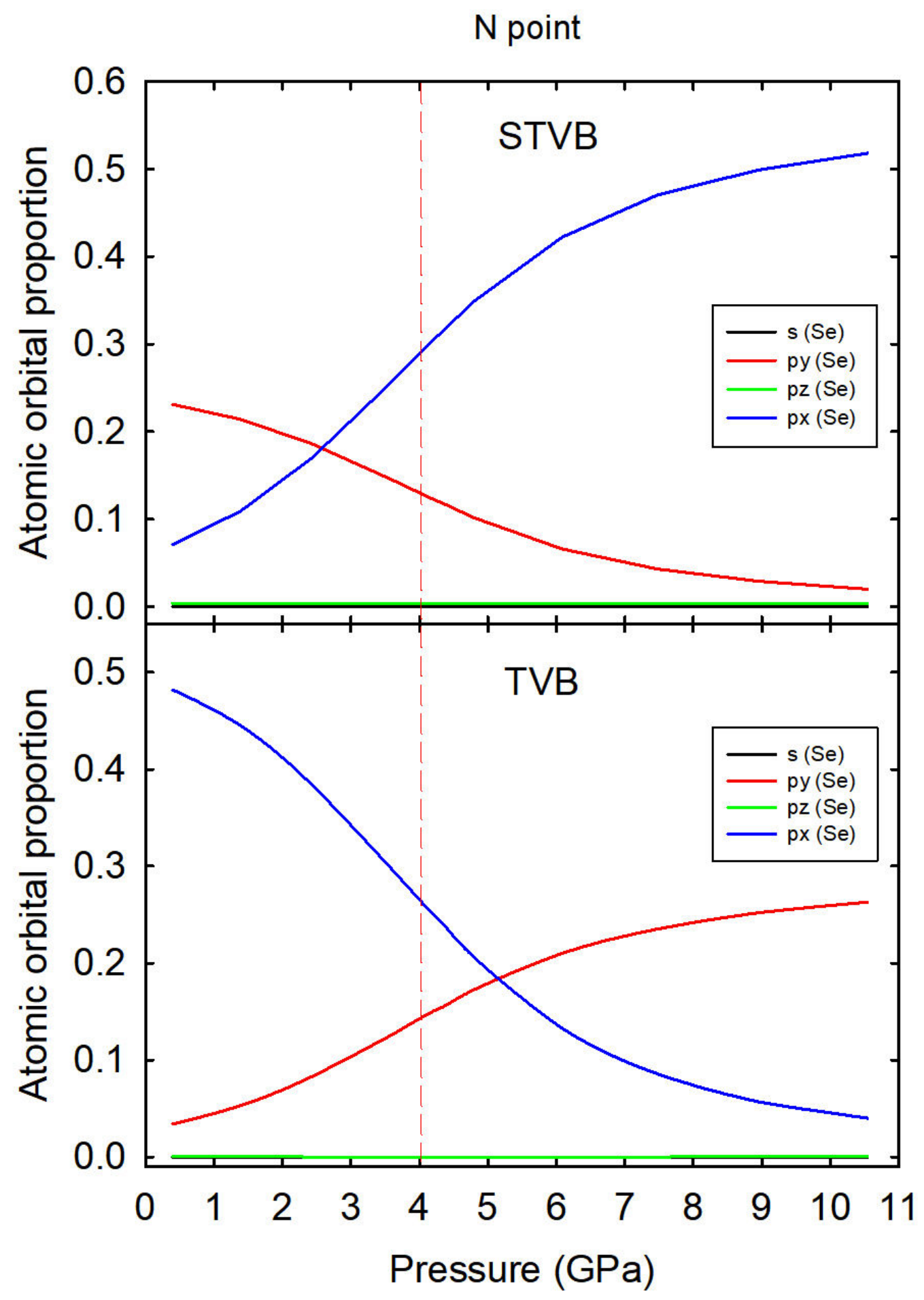




\section{TOC Graphic}

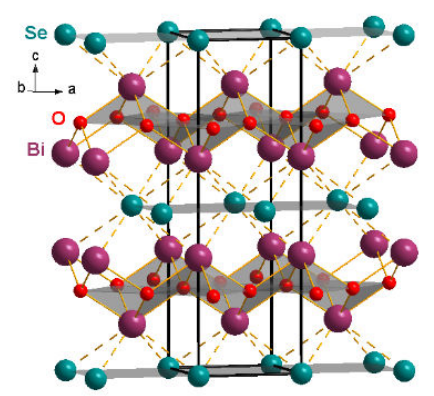




\title{
Supplementary Material
}

of

\section{Experimental and Theoretical Study of $\mathrm{Bi}_{2} \mathrm{O}_{2} \mathrm{SE}$ Under Compression}

\author{
A.L.J. Pereira, ${ }^{1,2^{*}}$ D. Santamaría-Pérez, ${ }^{3}$ J. Ruiz-Fuertes,${ }^{3,4}$ F.J. Manjón, ${ }^{1 *}$ V.P. Cuenca- \\ Gotor, ${ }^{1}$ R. Vilaplana, ${ }^{5}$ O. Gomis, ${ }^{5}$ C. Popescu, ${ }^{6}$ A. Muñoz, ${ }^{7}$ P. Rodríguez-Hernández, ${ }^{7}$ A. \\ Segura, ${ }^{3}$ L. Gracia, ${ }^{8}$ A. Beltrán, ${ }^{9}$ P. Ruleova,${ }^{10}$ C. Drasar ${ }^{10}$ and J. A. Sans ${ }^{1}$
}

${ }^{1}$ Instituto de Diseño para la Fabricación y Producción Automatizada, MALTA Consolider Team, Universitat Politècnica de València, València, Spain

${ }^{2}$ Grupo de Pesquisa de Materiais Fotonicos e Energia Renovável - MaFER, Universidade Federal da Grande Dourados, Dourados, MS, Brazil

${ }^{3}$ Departament de Física Aplicada - ICMUV, MALTA Consolider Team, Universitat de València, Burjassot, Spain

${ }^{4}$ DCITIMAC, MALTA Consolider Team, Universidad de Cantabria, Santander, Spain

${ }^{5}$ Centro de Tecnologías Físicas, MALTA Consolider Team, Universitat Politècnica de València, València, Spain

${ }^{6}$ CELLS-ALBA Synchrotron Light Facility, 08290 Cerdanyola, Barcelona, Spain

${ }^{7}$ Departamento de Física, Instituto de Materiales y Nanotecnología, MALTA Consolider Team, Universidad de La Laguna, Tenerife, Spain

${ }^{8}$ Departament de Química Física, MALTA Consolider Team, Universitat de València, Burjassot, Spain

${ }^{9}$ Departament de Quimica Física i Analitica, MALTA Consolider Team, Universitat Jaume I, Castellón, Spain

${ }^{10}$ Faculty of Chemical Technology, University of Pardubice, Pardubice, Czech Republic

*Corresponding authors, Email: andrepereira@ufgd.edu.br, fjmanjon@fis.upv.es 


\section{Structural data of $\mathrm{Bi}_{2} \mathrm{O}_{2} \mathrm{Se}$ under pressure}

Table S1. Lattice parameters and unit cell volumes of $\mathrm{Bi}_{2} \mathrm{O}_{2} \mathrm{Se}$ obtained from synchrotron XRD experiments at different pressures.

\begin{tabular}{|c|c|c|c|}
\hline $\mathrm{P}(\mathrm{GPa})$ & a axis $(\AA)$ & $\mathrm{c}$ axis $(\AA)$ & $\mathrm{V}\left(\AA^{3}\right)$ \\
\hline $0.49(3)$ & $3.8823(8)$ & $12.184(3)$ & $183.65(9)$ \\
\hline $0.68(3)$ & $3.8792(7)$ & $12.167(3)$ & $183.10(8)$ \\
\hline $1.23(3)$ & $3.8718(6)$ & $12.126(2)$ & $181.79(7)$ \\
\hline $1.47(3)$ & $3.8683(6)$ & $12.106(2)$ & $181.16(7)$ \\
\hline $1.97(4)$ & $3.8620(5)$ & $12.072(2)$ & $180.05(5)$ \\
\hline $2.59(3)$ & $3.8543(6)$ & $12.032(3)$ & $178.75(7)$ \\
\hline $3.00(4)$ & $3.8498(4)$ & $12.004(2)$ & $177.91(4)$ \\
\hline $3.57(5)$ & $3.8422(6)$ & $11.967(2)$ & $176.66(6)$ \\
\hline $5.38(6)$ & $3.8231(7)$ & $11.871(3)$ & $173.50(8)$ \\
\hline $6.01(5)$ & $3.8159(5)$ & $11.832(2)$ & $172.29(7)$ \\
\hline $6.99(6)$ & $3.8062(5)$ & $11.785(2)$ & $170.73(5)$ \\
\hline $7.99(7)$ & $3.7982(17)$ & $11.738(6)$ & $169.34(17)$ \\
\hline $8.5(1)$ & $3.793(2)$ & $11.710(8)$ & $168.5(2)$ \\
\hline $9.6(2)$ & $3.788(3)$ & $11.675(12)$ & $167.5(3)$ \\
\hline $11.9(2)$ & $3.7786(18)$ & $11.629(7)$ & $166.03(19)$ \\
\hline $12.4(2)$ & $3.7754(19)$ & $11.611(8)$ & $165.5(2)$ \\
\hline $13.2(2)$ & $3.7724(15)$ & $11.591(9)$ & $164.95(19)$ \\
\hline $14.5(3)$ & $3.770(3)$ & $11.567(12)$ & $164.4(3)$ \\
\hline $15.2(2)$ & $3.767(3)$ & $11.549(13)$ & $163.9(3)$ \\
\hline $15.8(3)$ & $3.763(3)$ & $11.526(13)$ & $163.2(3)$ \\
\hline $16.3(3)$ & $3.761(3)$ & $11.507(13)$ & $162.8(3)$ \\
\hline $18.2(4)$ & $3.753(4)$ & $11.465(15)$ & $161.4(4)$ \\
\hline $19.9(4)$ & $3.744(3)$ & $11.422(16)$ & $160.1(4)$ \\
\hline $21.7(4)$ & $3.735(4)$ & $11.382(19)$ & $158.8(4)$ \\
\hline & & &
\end{tabular}




\section{Vibrational modes in $\mathrm{Bi}_{2} \mathrm{O}_{2} \mathrm{Se}$ at the $\Gamma$ point}

$\mathrm{Bi}_{2} \mathrm{O}_{2} \mathrm{Se}$ crystallizes in the tetragonal body-centered $14 / \mathrm{mmm}$ structure and has two formula units in the primitive cell, therefore, it has 10 normal vibrational modes at $\Gamma$ whose mechanical decomposition is ${ }^{1}$ :

$$
\Gamma=1 A_{1 g}(R)+2 A_{2 u}(I R)+1 B_{1 g}(R)+2 E_{u}(I R)+2 E_{g}(R)+A_{2 u}+E_{u}
$$

where $A_{1 g}, B_{1 g}$ and $E_{g}$ modes are Raman-active $(R)$ and $A_{2 u}$ and $E_{u}$ are infrared-active (IR). In total, there are four Raman-active modes $\left(\Gamma_{\text {Raman }}=A_{1 g}+B_{1 g}+2 E_{g}\right)$, four infrared-active (IR) modes $\left(\Gamma_{I R}=2 \mathrm{~A}_{2 \mathrm{u}}+2 \mathrm{E}_{\mathrm{u}}\right)$ and two acoustic modes $\left(\Gamma_{\text {Acoustic }}=\mathrm{A}_{2 \mathrm{u}}+\right.$ $\mathrm{E}_{\mathrm{u}}$ ). It must be mentioned that that $\mathrm{E}$ modes are doubly degenerated. The assignment of the vibrational modes to atomic movements can be done thanks to the program J-ICE ${ }^{2}$ that reads OUTCAR files of VASP.
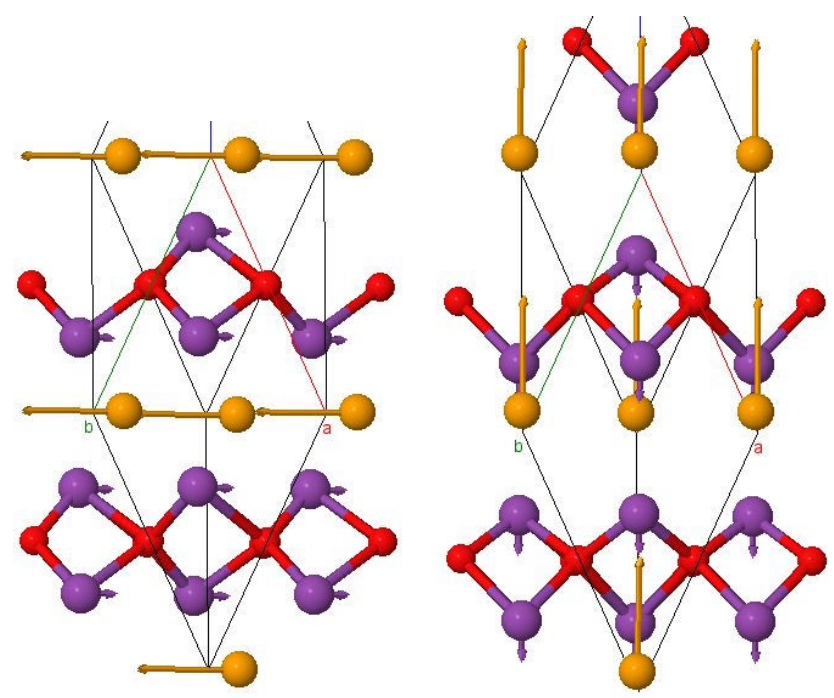

Figure S1. Atomic movements of low-frequency interlayer vibrational modes $\mathbf{E}_{u}$ and $\mathbf{A}_{2 u} \cdot$ 

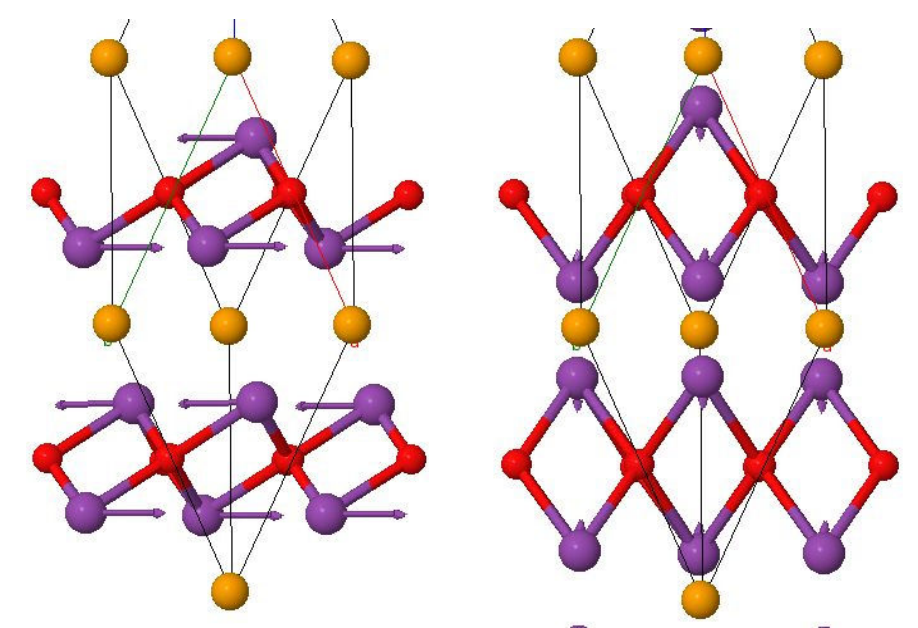

Figure S2. Atomic movements of low-frequency interlayer $\mathbf{E}_{g}$ and $A_{1 g}$ vibrational modes.

It is well-known that in layered materials, which usually crystallize either in a hexagonal or tetragonal space group, the lowest-frequency E (doubly degenerated) and A (or B) modes at the $\Gamma$ point can be classified as interlayer (out-of-phase vibrations of atoms corresponding to adjacent layers) or intralayer (out-of-phase vibrations of atoms inside the layers) modes. Interlayer E and A (or B) modes are usually related to shear or transversal vibrations between adjacent layers along the layer plane $(a-b)$ and to longitudinal vibrations of one layer against the adjacent ones (along the $c$ axis), respectively. Both $\mathrm{E}$ and $\mathrm{A}$ (or $\mathrm{B}$ ) interlayer modes come from transversal acoustic (TA) and longitudinal acoustic (LA) modes, respectively, due to the folding of points of the Brillouin zone border into the $\Gamma$ point due to the decreasing symmetry from cubic to hexagonal or tetragonal. Similarly, E and A (or B) intralayer modes come from transversal optic (TO) and longitudinal optic (LO) modes at $\Gamma$ and from additional modes due to the folding of the BZ border into the $\Gamma$ point.

The number of interlayer and intralayer modes in layered materials depends on the complexity of the unit cell. In the simplest case, there should be two interlayer modes and four intralayer modes. In the case of $\mathrm{Bi}_{2} \mathrm{O}_{2} \mathrm{Se}$, there are four interlayer modes $\left(E_{\mathrm{u}}, \mathrm{A}_{2 \mathrm{u}}, \mathrm{E}_{\mathrm{g}}\right.$ and $\left.\mathrm{A}_{1 \mathrm{~g}}\right)$ having the lowest frequencies, while the four intralayer modes $\left(\mathrm{E}_{\mathrm{u}}\right.$, $B_{g}, A_{2 u}$ and $E_{g}$ ) have the highest frequencies. The two modes with lowest frequency, $E_{u}$ and $\mathrm{A}_{2 \mathrm{u}}$, are IR-active and correspond to out-of-phase movements of Se and Bi atoms (see Fig. S1); i.e. both modes are typical interlayer modes of layered structures. In fact, they correspond to the shear mode between $\mathrm{Bi}_{2} \mathrm{O}_{2}$ and Se layers in the layer plane and to 
the longitudinal vibration of the $\mathrm{Bi}_{2} \mathrm{O}_{2}$ and Se layers one against the other along the $c$ axis (perpendicular to the layer plane). On the other hand, the low-frequency $E_{g}$ and $A_{1 g}$ modes are Raman-active and correspond to out-of-phase Bi movements in the layer plane and perpendicular to the layer plane, respectively (see Fig. S2). These two modes are not so typical of layered materials and come from the fact that there are two $\mathrm{Bi}_{2} \mathrm{O}_{2}$ layers in the unit cell so that these two modes can be considered as the complementary movements of $\mathrm{Bi}$ atoms in the two low-frequency $\mathrm{E}_{\mathrm{u}}$ and $\mathrm{A}_{2 \mathrm{u}}$ modes. In these two modes, there is an in-phase movement of $\mathrm{Bi}$ atoms in a $\mathrm{Bi}_{2} \mathrm{O}_{2}$ layer, while in the two low-frequency $\mathrm{E}_{\mathrm{g}}$ and $\mathrm{A}_{\mathrm{lg}}$ modes there is an out-of-phase movement of $\mathrm{Bi}$ atoms in the layer. Therefore, the two low-frequency $E_{u}$ and $A_{2 u}$ modes are proper interlayer modes between $\mathrm{Bi}_{2} \mathrm{O}_{2}$ and Se layers, while the two low-frequency $\mathrm{E}_{\mathrm{g}}$ and $\mathrm{A}_{1 \mathrm{~g}}$ modes are half interlayer modes between $\mathrm{Bi}_{2} \mathrm{O}_{2}$ and $\mathrm{Se}$ layers and half interlayer modes between the upper and lower halves of each $\mathrm{Bi}_{2} \mathrm{O}_{2}$ layer.

In layered compounds with typical van der Waals gap between the layers, the low-frequency interlayer shear mode exhibits a much smaller pressure coefficient than other modes, whereas the low-frequency A (or B) mode displays the largest pressure coefficient. For example, the E and A modes with frequencies around $40(60) \mathrm{cm}^{-1}$ and $116(133) \mathrm{cm}^{-1}$ in InSe (GaSe) have pressure coefficients of $0.68(0.85) \mathrm{cm}^{-1} / \mathrm{GPa}$ and $5.41(5.78) \mathrm{cm}^{-1} / \mathrm{GPa}$, respectively ${ }^{3,4}$. Usually, the small pressure coefficient of the lowfrequency $\mathrm{E}$ mode in layered materials is ascribed to the weak bending force constant due to weak van der Waals forces between the neighboring layers. On the other hand, the large pressure coefficient of the low-frequency A mode is due to the extraordinary increase of the stretching force constant between neighboring layers due to the strong decrease of the interlayer distance $\mathrm{e}^{3,4}$. A similar behavior is found in layered topological insulators $\mathrm{Bi}_{2} \mathrm{Se}_{3}, \mathrm{Bi}_{2} \mathrm{Te}_{3}$ and $\mathrm{Sb}_{2} \mathrm{Te}_{3}{ }^{5-7}$. However, a different behavior was recently observed in layered BiTeBr and BiTeI, which also feature a van der Waals gap between the layers ${ }^{8}$. In these semiconductors, the low-frequency $E_{1}(\mathrm{TO})$ mode has a similar pressure coefficient ( 4.3 and $3.5 \mathrm{~cm}^{-1} / \mathrm{GPa}$, respectively) than the rest of the optic modes and the low-frequency $\mathrm{A}_{1}(\mathrm{TO})$ mode has not so large pressure coefficient (4.5 and 4.6 $\mathrm{cm}^{-1} / \mathrm{GPa}$, respectively) as expected for a van der Waals compound. This result suggests that interlayer forces in these two compounds are stronger than common van der Waals forces in other layered compounds, likely due to the asymmetry of the layers what causes a strong polarity of bismuth tellurohalides ${ }^{9}$. Moreover, the rather similar pressure 
coefficients of these two modes in BiTeBr and BiTeI also suggests that bending and stretching interlayer bonds tend to harden at similar rates with pressure in both compounds; i.e., the anisotropy in the properties along the layers and perpendicular to the layers is not so high as in other layered compounds and tend to disappear at a similar rate with increasing pressure in both compounds.

The case of $\mathrm{Bi}_{2} \mathrm{O}_{2} \mathrm{Se}$ is similar to that of $\mathrm{BiTeBr}$ and $\mathrm{BiTeI}$ because both interlayer $\mathrm{E}_{\mathrm{u}}$ and $\mathrm{A}_{2 \mathrm{u}}$ modes show pressure coefficients of 5.5 and $4.1 \mathrm{~cm}^{-1} / \mathrm{GPa}$, respectively. Figure $\mathbf{S 3}$ shows the pressure dependence of the theoretical IR-active modes of $\mathrm{Bi}_{2} \mathrm{O}_{2} \mathrm{Se}$. Therefore, the same explanation already given for the interlayer modes in $\mathrm{BiTeBr}$ and $\mathrm{BiTeI}$ seems to be valid for $\mathrm{Bi}_{2} \mathrm{O}_{2}$ Se; i.e. interlayer bonding forces are of the same order or stronger that in polar van der Waals compounds, like BiTeBr and BiTeI, and clearly much larger than in non-polar van der Waals compounds, like GaSe and InSe. Curiously, the two interlayer $\mathrm{E}_{\mathrm{g}}$ and $\mathrm{A}_{1 \mathrm{~g}}$ modes in $\mathrm{Bi}_{2} \mathrm{O}_{2} \mathrm{Se}$ have pressure coefficients of 1.1 and $2.1 \mathrm{~cm}^{-1} / \mathrm{GPa}$. In this case, the shear mode has a small pressure coefficient as in van der Waals compounds, but the longitudinal mode has a very small pressure coefficient as compared to van der Waals compounds. In summary, we can say that both shear interlayer modes $\left(E_{u}\right.$ and $\left.E_{g}\right)$ and longitudinal interlayer modes $\left(\mathrm{A}_{2 \mathrm{u}}\right.$ and $\left.\mathrm{A}_{1 \mathrm{~g}}\right)$ have an average pressure coefficient around $3.2 \mathrm{~cm}^{-1} / \mathrm{GPa}$ in $\mathrm{Bi}_{2} \mathrm{O}_{2} \mathrm{Se}$, thus suggesting that this is a $2 \mathrm{D}$ material with bonding interlayer forces between $\mathrm{Bi}_{2} \mathrm{O}_{2}$ and $\mathrm{Se}$ layers considerably stronger than common van der Waals layered compounds. 


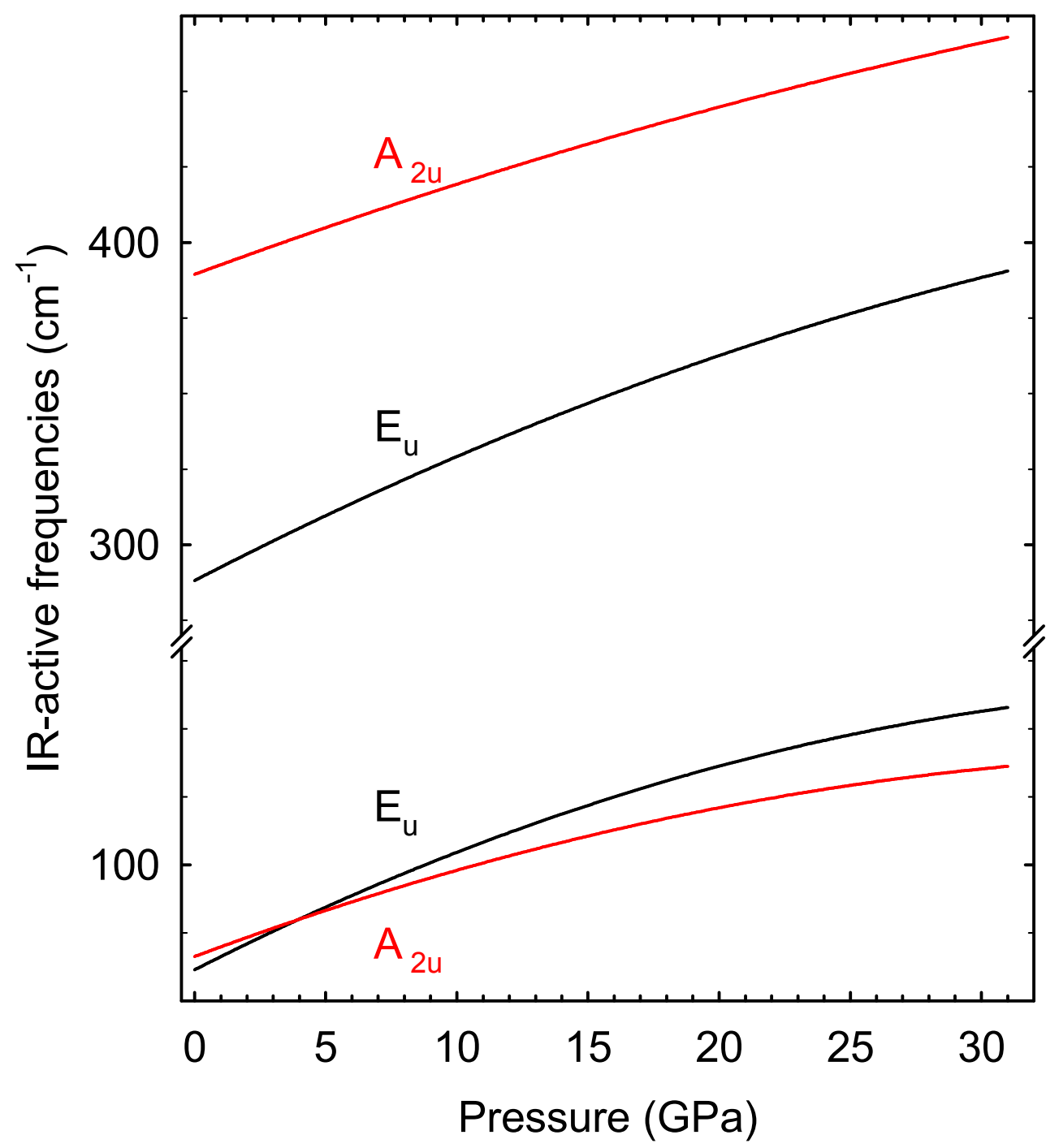

Figure S3. Theoretical pressure dependence of the IR-active modes of $\mathrm{Bi}_{2} \mathrm{O}_{2} \mathrm{Se}$. Different colors represent IR-active modes of different symmetries. 

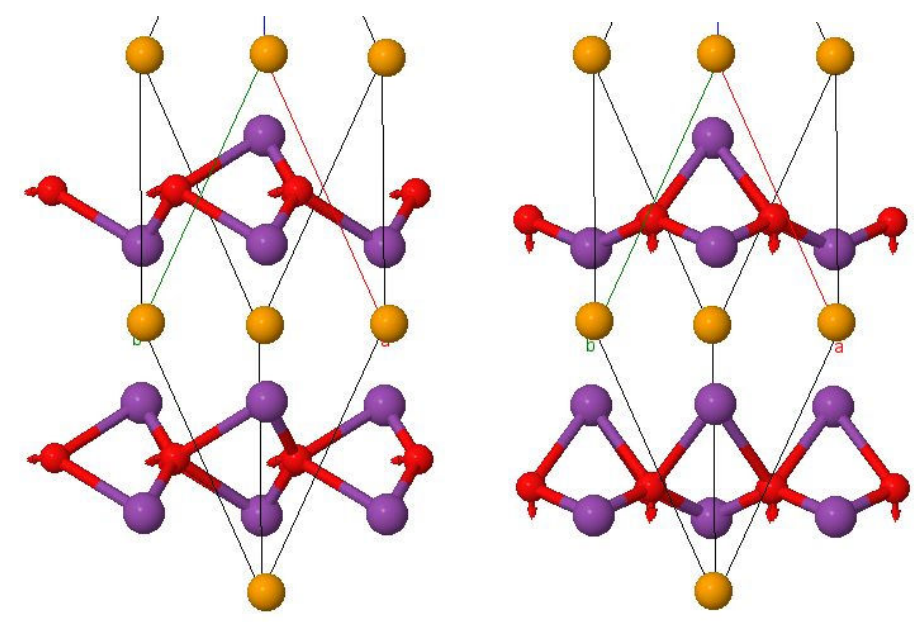

Figure S4. Atomic movements of high-frequency intralayer $E_{u}$ and $A_{2 u}$ vibrational modes.
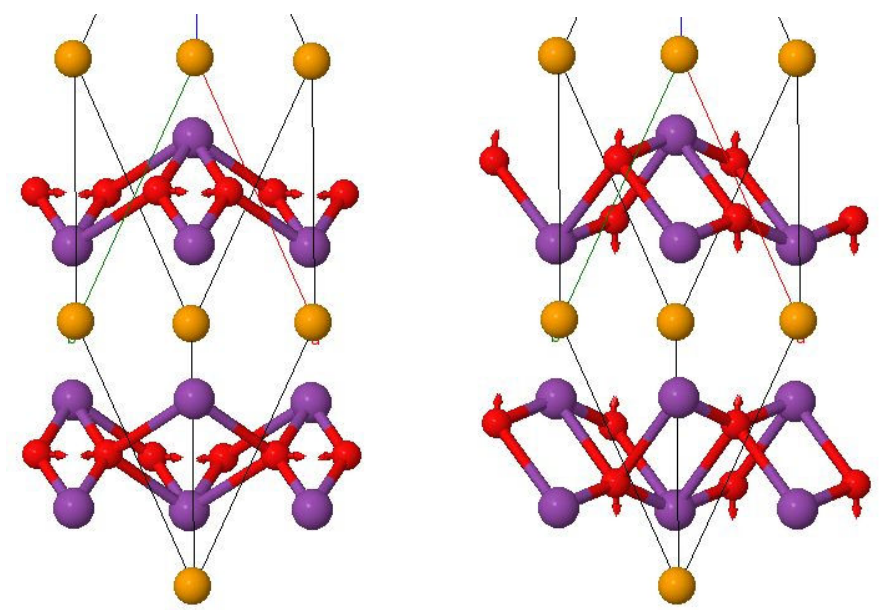

Figure S5. Atomic movements of high-frequency intralayer $E_{g}$ and $B_{g}$ vibrational modes.

As regards the high-frequency modes in $\mathrm{Bi}_{2} \mathrm{O}_{2} \mathrm{Se}$, they are intralayer modes related to atomic vibrations of $\mathrm{O}$ atoms inside the $\mathrm{Bi}_{2} \mathrm{O}_{2}$ layers. The two high-frequency $E_{u}$ and $A_{2 u}$ modes are IR-active and correspond to in-phase movements of $\mathrm{O}$ atoms in the layer plane and perpendicular to the layer plane, respectively (see Fig. S4) and $\mathrm{E}_{\mathrm{g}}$ and $\mathrm{B}_{\mathrm{g}}$ modes are Raman-active and correspond to out-of-phase movements of $\mathrm{O}$ atoms inside the $\mathrm{Bi}_{2} \mathrm{O}_{2}$ layer in the layer plane and perpendicular to the layer plane, respectively (see Fig. S5). As regards the pressure coefficients of all these intralayer modes, they are between 3.0 and $5.3 \mathrm{~cm}^{-1} / \mathrm{GPa}$ which are typical of bending and stretching modes of ionic-covalent bonds as the $\mathrm{Bi}-\mathrm{O}$ bonds in $\mathrm{Bi}_{2} \mathrm{O}_{3}{ }^{10,11}$. In fact, the average pressure coefficient of the transversal and longitudinal modes is 3.7 and $2.9 \mathrm{~cm}^{-}$ 
${ }^{1} / \mathrm{GPa}$, which are values that are in good agreement with the largest value of transversal than longitudinal modes in most ionic-covalent semiconductors ${ }^{12}$.

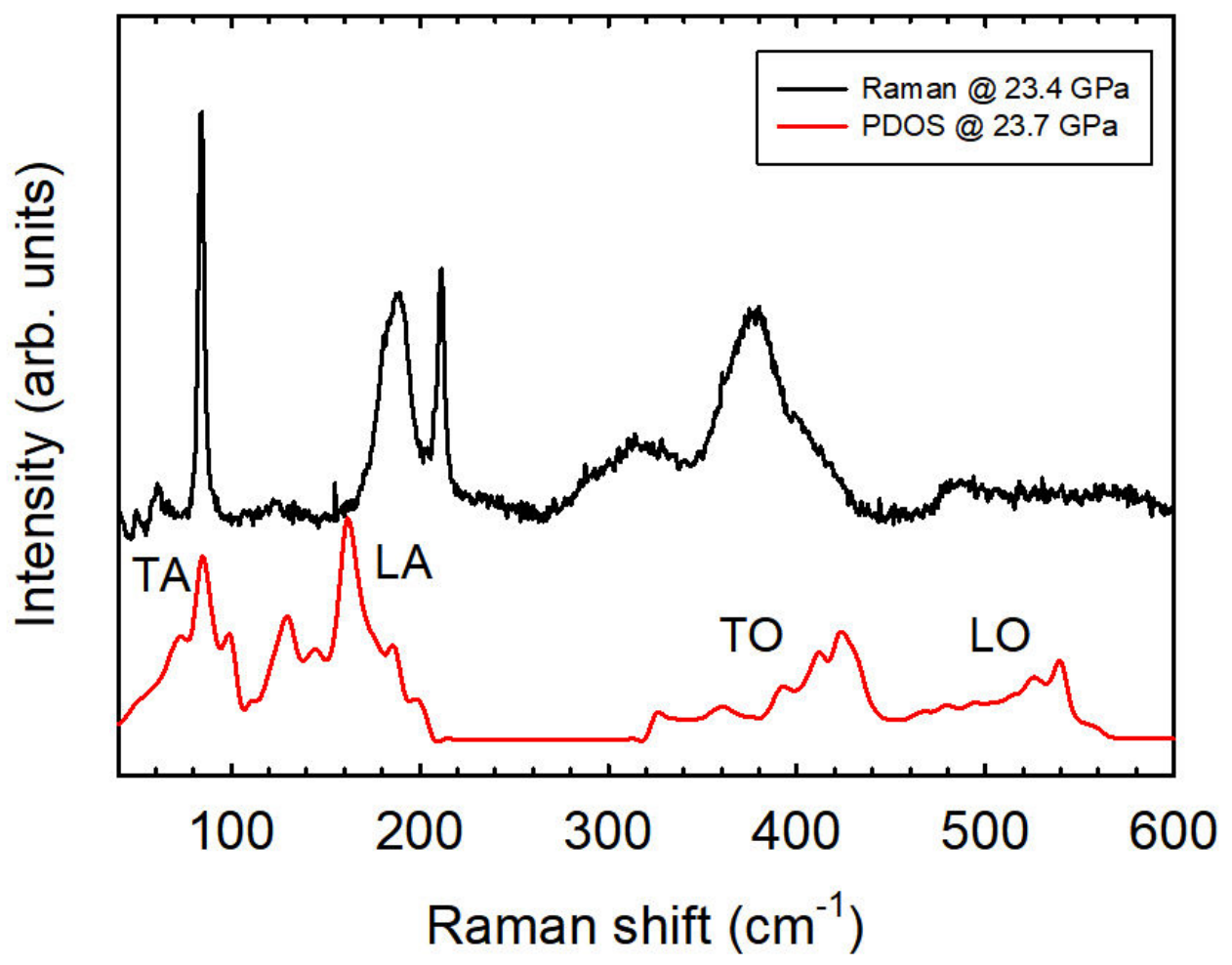

Figure S6. Comparison of the Raman spectrum of $\mathrm{Bi}_{2} \mathrm{O}_{2} \mathrm{Se}$ at $23.4 \mathrm{GPa}$ and the theoretical one-phonon density of states at 23.7 GPa.

The RS spectrum of $\mathrm{Bi}_{2} \mathrm{O}_{2} \mathrm{Se}$ at $23 \mathrm{GPa}$ shows the two narrow first-order Raman peaks $\mathrm{E}_{\mathrm{g}}$ and $\mathrm{A}_{1 \mathrm{~g}}$ near 85 and $215 \mathrm{~cm}^{-1}$, respectively. The comparison of RS spectra at $23 \mathrm{GPa}$ with the one-phonon density of states (PDOS) suggests that the other broad bands could originate from one-phonon density of states due to defect assisted Raman scattering. In particular, the broad band at 180-190 $\mathrm{cm}^{-1}$ could be assigned to the scattering from the top phonon band of the acoustic region (below $210 \mathrm{~cm}^{-1}$ ) that corresponds to the longitudinal acoustic (LA) region in binary semiconductors. On the other hand, the broad band between 260 and $450 \mathrm{~cm}^{-1}$ could be likely assigned to the scattering of the optic transveral (TO) region and other weaker bands at higher frequencies could be likely due to the scattering of the optic longitudinal (LO) region. No clear sign of the PDOS related to the TA region seems to be observed in the RS spectrum. 


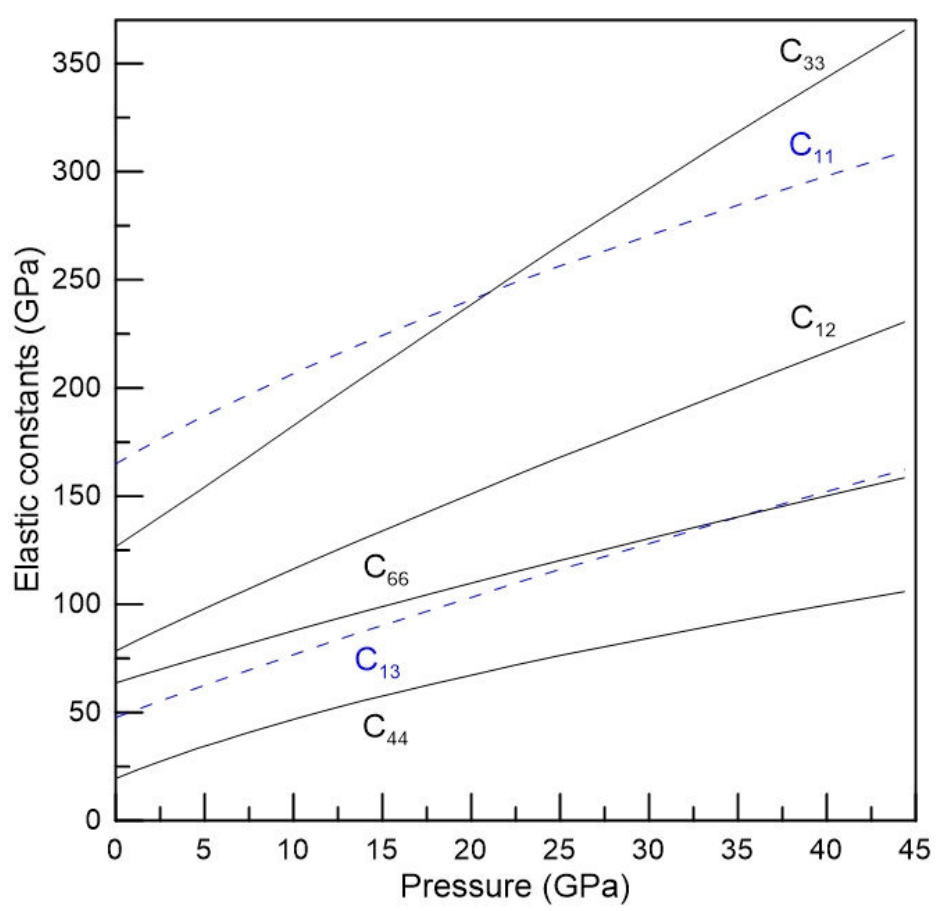

Figure S7. Pressure dependence of theoretical elastic constants, $C_{\mathrm{ij}}$, calculated with VASP in $\mathrm{Bi}_{2} \mathrm{O}_{2} \mathrm{Se}$.

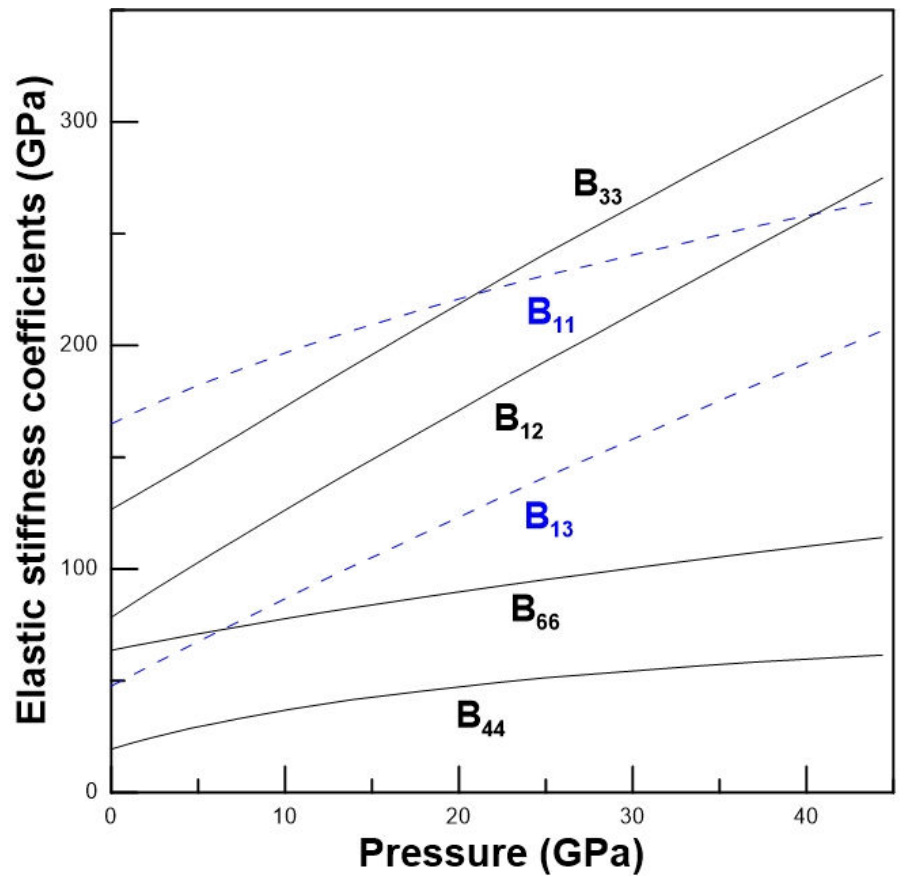

Figure S8. Pressure dependence of theoretical elastic stiffness coefficients, $B_{i j}$, calculated with VASP in $\mathrm{Bi}_{2} \mathrm{O}_{2} \mathrm{Se}$.

The generalized stability criteria for a body-centered tetragonal lattice ${ }^{13,14}$ are given by: 


$$
\begin{aligned}
& M_{1}=C_{11}-P>0 \\
& M_{2}=C_{11}-C_{12}-2 P>0 \\
& M_{3}=\left(C_{33}-P\right)\left(C_{11}+C_{12}\right)-2\left(C_{13}+P\right)^{2}>0 \\
& M_{4}=C_{44}-P>0 \\
& M_{5}=C_{66}-P>0
\end{aligned}
$$




\section{Density of States of $\mathrm{Bi}_{2} \mathrm{O}_{2} \mathrm{Se}$}

Electronic Density of States

$\mathrm{Bi} 2 \mathrm{O} 2 \mathrm{Se}$

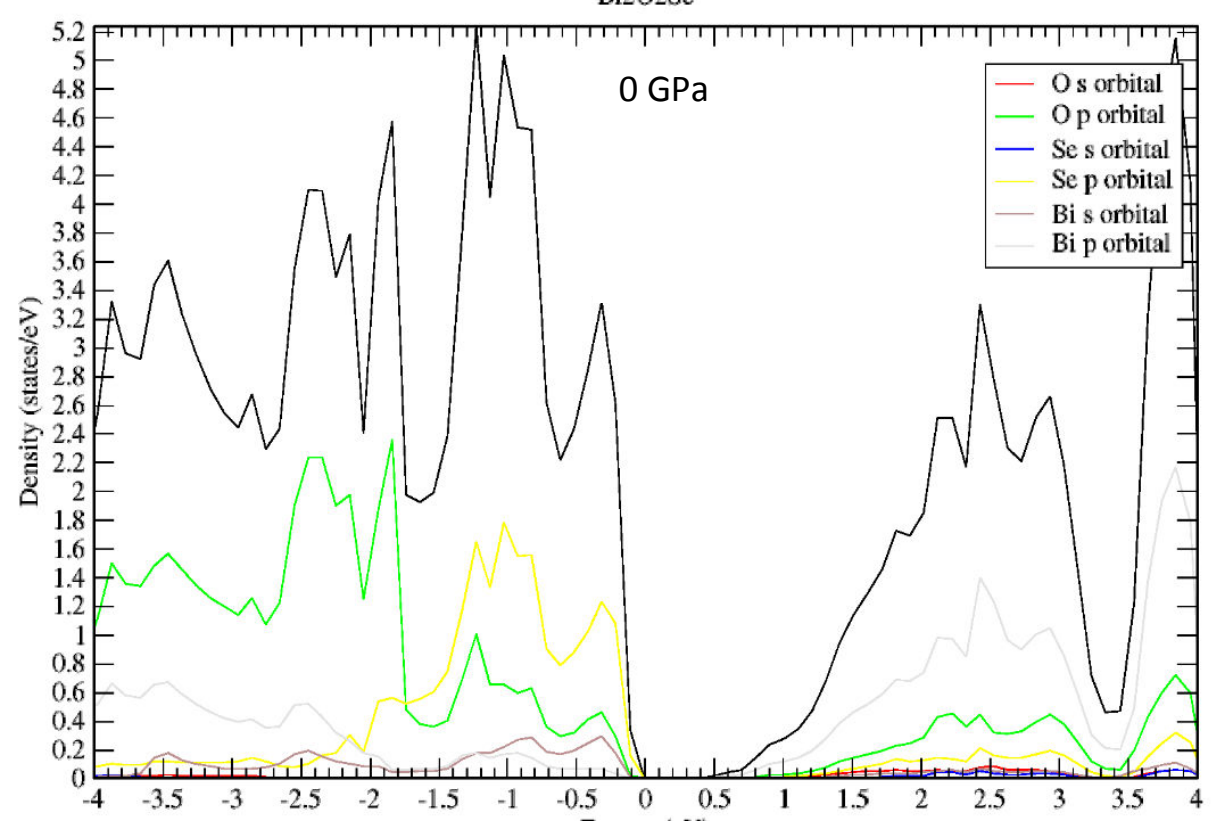

Electronic Density of States

$\mathrm{Bi} 2 \mathrm{O} 2 \mathrm{Se}$

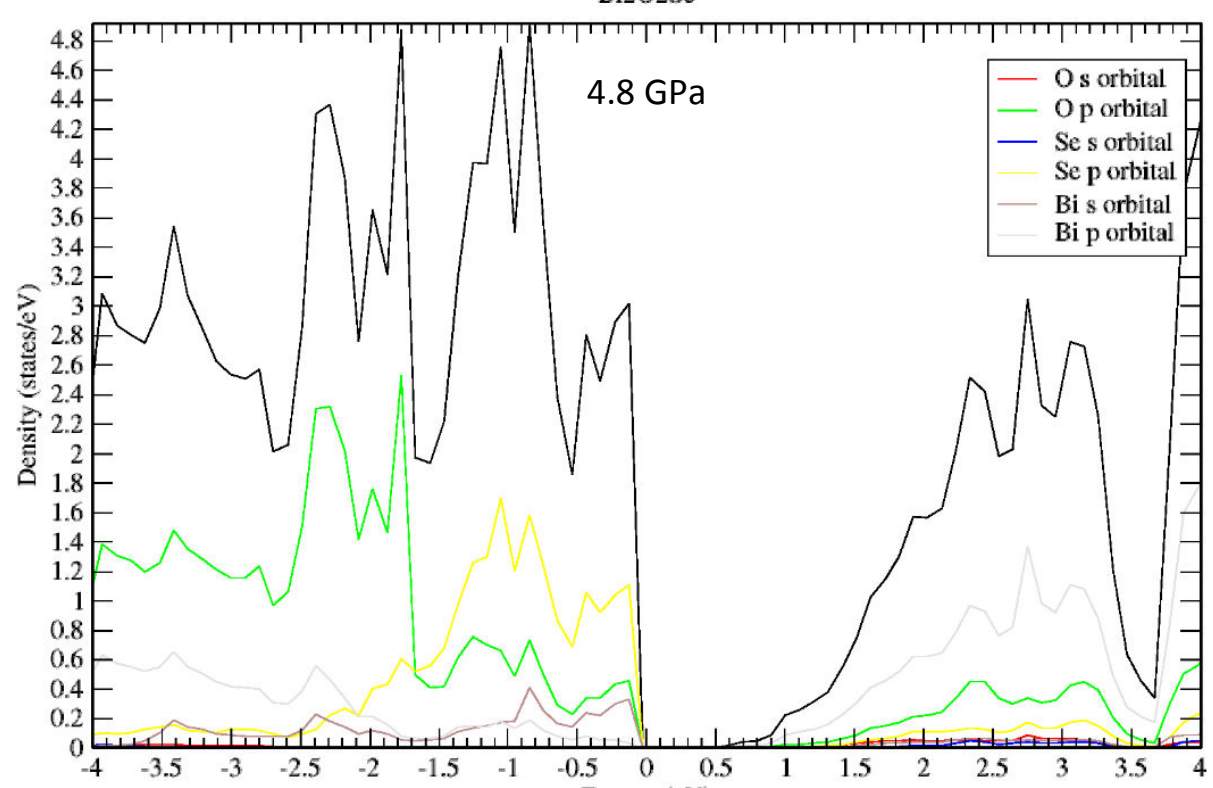

Figure S9. Electronic density of states (DOS) at 0 and 4.8 GPa showing the change of the bandgap with pressure in $\mathrm{Bi}_{2} \mathrm{O}_{2} \mathrm{Se}$. 


\section{References}

1. Kroumova, E.; Aroyo, M. I.; Perez Mato, J. M.; Kirov, A.; Capillas, C.; Ivantchev, S.; Wondratschek, H. Bilbao Crystallographic Server: Useful Databases and Tools for Phase Transitions Studies. Phase Trans. 2003, 76, 155170.

2. Canepa, P.; Hanson, R. M.; Ugliengo, P.; Alfredsson, M. J-ICE: A New Jmol Interface for Handling and Visualizing Crystallographic and Electronic Properties. J. Appl. Cryst. 2011, 44, 225-229.

3. Ulrich, C.; Mroginski, M. A.; Goñi, A. R.; Cantarero, A.; Schwarz, U.; Muñoz, V.; Syassen, K. Vibrational Properties of InSe under Pressure: Experiment and Theory. Phys. Stat. Sol. (b) 1996, 198, 121-127.

4. Kulibekov, A. M.; Olijnyk, H. P.; Jephcoat, A. P.; Salaeva, Z. Y.; Onari, S.; Allakverdiev, K. R. Raman Scattering under Pressure and the Phase Transition in $\varepsilon$-GaSe. Phys. Stat. Sol (b) 2003, 235, 517-520.

5. Vilaplana, R.; Gomis, O.; Manjón, F. J.; Segura, A.; Pérez-González, E.; RodríguezHernández, P.; Muñoz, A.; González, J.; Marín-Borrás, V.; MuñozSanjosé, V.; et al. High-Pressure Vibrational and Optical Study of $\mathrm{Bi}_{2} \mathrm{Te}_{3}$. Phys. Rev. B 2011, 84, 104112.

6. Gomis, O.; Vilaplana, R.; Manjón, F. J.; Rodríguez-Hernández, P.; PérezGonzález, E.; Muñoz, A.; Kucek, V.; Drasar, C. Lattice Dynamics of $\mathrm{Sb}_{2} \mathrm{Te}_{3}$ at High Pressures. Phys. Rev. B 2011, 84, 174305.

7. Vilaplana, R.; Santamaría-Pérez, D.; Gomis, O.; Manjón, F. J.; González, J.; Segura, A.; Muñoz, A.; Rodríguez-Hernández, E.; Pérez-González, E.; MarínBorrás, V.; et al. Structural and Vibrational Study of $\mathrm{Bi}_{2} \mathrm{Se}_{3}$ under High Pressure. Physical Review B 2011, 84, 184110. 
8. Sans, J. A.; Manjón, F. J.; Pereira, A. L. J.; Vilaplana, R.; Gomis, O.; Segura, A.; Muñoz, A.; Rodríguez-Hernández, P.; Popescu, C.; Drasar, C.; et al. Structural, Vibrational, and Electrical Study of Compressed BiTeBr. Phys. Rev. B 2016, 93, 024110 .

9. Ma, Y. D.; Dai, Y.; Wei, W.; Li, X. R.; Huang, B. B. Emergence of Electric Polarity in $\operatorname{BiTeX}(\mathrm{X}=\mathrm{Br}$ and $\mathrm{I})$ Monolayers and the Giant Rashba Spin Splitting. Phys. Chem. Chem. Phys. 2014, 16, 17603-17609.

10. Pereira, A. L. J.; Gomis, O.; Sans, J. A.; Pellicer-Porres, J.; Manjón, F. J.; Beltran, A.; Rodríguez-Hernandez, P.; Muñoz, A. Pressure Effects on the Vibrational Properties of $\alpha-\mathrm{Bi}_{2} \mathrm{O}_{3}$ : An Experimental and Theoretical Study. $J$. Phys.: Cond. Mat. 2014, 26, 225401.

11. Pereira, A. L. J.; Sans, J. A.; Vilaplana, R.; Gomis, O.; Manjón, F. J.; RodríguezHernández, P.; Muñoz, A.; Popescu, C.; Beltrán, A. Isostructural Second-Order Phase Transition of $\beta-\mathrm{Bi}_{2} \mathrm{O}_{3}$ at High Pressures: An Experimental and Theoretical Study. J. Phys. Chem. C 2014, 118, 23189-23201.

12. Manjón, F. J.; Syassen, K.; Lauck, R. Effect of Pressure on Phonon Modes in Wurtzite Zinc Oxide. High Pressure Research 2002, 22, 299-304.

13. Wallace, D. C. Thermoelasticity of Stressed Materials and Comparison of Various Elastic Constants. Phys. Rev. 1967, 162, 776-789.

14. Grimvall, G.; Magyari-Köpe, B.; Ozolinš, V.; Persson, K. A. Lattice Instabilities in Metallic Elements. Rev. Mod. Phys. 2012, 84, 945-986. 